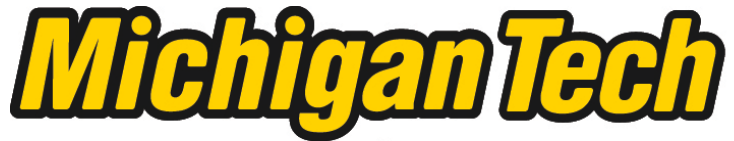 \\ Michigan Technological University Create the Future Digital Commons @ Michigan Tech
}

Dissertations, Master's Theses and Master's Reports - Open

Dissertations, Master's Theses and Master's

Reports

2012

\section{Charge and spin transport in nanoscale junction from first principles}

Subhasish Mandal

Michigan Technological University

Follow this and additional works at: https://digitalcommons.mtu.edu/etds

Part of the Physics Commons

Copyright 2012 Subhasish Mandal

\section{Recommended Citation}

Mandal, Subhasish, "Charge and spin transport in nanoscale junction from first principles", Dissertation, Michigan Technological University, 2012.

https://doi.org/10.37099/mtu.dc.etds/111

Follow this and additional works at: https://digitalcommons.mtu.edu/etds

Part of the Physics Commons 
CHARGE AND SPIN TRANSPORT IN NANOSCALE JUNCTION FROM FIRST

\title{
PRINCIPLES
}

\author{
By \\ Subhasish Mandal

\begin{abstract}
A DISSERTATION
Submitted in partial fulfillment of the requirements for the degree of DOCTOR OF PHILOSOPHY

(Engineering Physics)
\end{abstract}

MICHIGAN TECHNOLOGICAL UNIVERSITY

2012

(c) 2012 Subhasish Mandal 

This dissertation, "Charge and Spin Transport in Nanoscale Junction from First Principles," is hereby approved in partial fulfillment of the requirements for the Degree of DOCTOR OF PHILOSOPHY IN ENGINEERING PHYSICS.

Department of Physics

Signatures:

Dissertation Advisor

Dr. Ranjit Pati

Committee Member

Dr. Maximilian Seel

Committee Member

Dr. Ravindra Pandey

Committee Member

Dr. Gregory Odegard

Department Chair

Dr. Ravindra Pandey

Date 

To my lovely family ... 



\section{Contents}

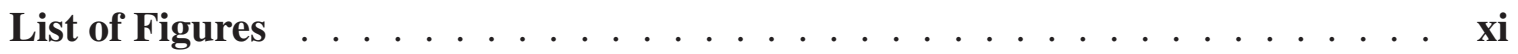

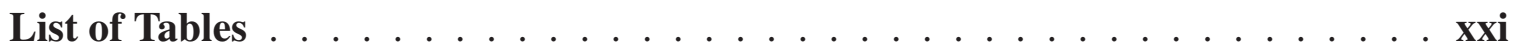

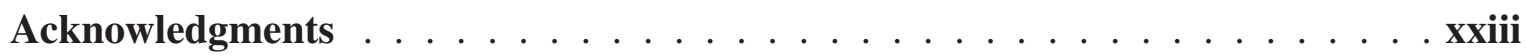

Abstract $\ldots \ldots \ldots \ldots \ldots \ldots \ldots \ldots \ldots \ldots \ldots \ldots \ldots \ldots \ldots \ldots$

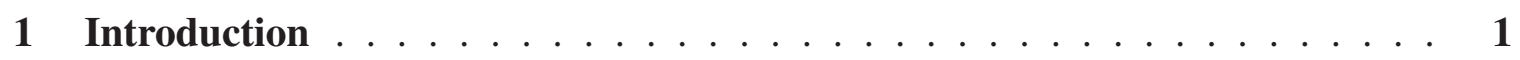

2 Density Functional Theory $\ldots \ldots \ldots \ldots \ldots \ldots$

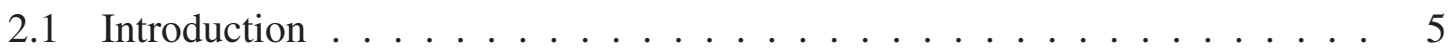

2.2 Many Electron Hamiltonian $\ldots \ldots \ldots \ldots$

2.3 Hatree-Fock Method . . . . . . . . . . . . . . . . . 8

2.4 Energy in Terms of Density . . . . . . . . . . . . . . . 10

2.5 The Hohenberg-Kohn Theorem . . . . . . . . . . . . . . . . . . . 12

2.6 Kohn-Sham Method . . . . . . . . . . . . . . . . . . 15

2.6.1 Local Density Approximation _ . . . . . . . . . . . . 17

2.6.2 Generalized Gradient Approximation (GGA) $\ldots \ldots \ldots$ 
2.6 .3 B3LYP . . . . . . . . . . . . . . . 20

3 Theory of Quantum Transport for Nanoscale Systems . . . . . . . . . . . . 21

3.1 Introduction . . . . . . . . . . . . . . . 21

3.2 Modeling the Device . . . . . . . . . . . . . . . 22

3.2.1 Looking Back from the Uncertainty Principles . . . . . . . . . . . . 24

3.3 Landauer Approach . . . . . . . . . . . . . . . . . . 25

3.4 Green's Function and Self Energy . . . . . . . . . . . . . . . . 29

3.5 Spin-dependent Transport . . . . . . . . . . . . . . . . . 34

4 Charge Transport in a Zero-dimensional Codoped Molecular Junction . . . 39

4.1 Motivation . . . . . . . . . . . . . . . . 39

4.2 Codoping Approach in a Single Molecular Junction . . . . . . . . . . . . 41

4.3 Computational Methods . . . . . . . . . . . . . . . . . . 42

4.4 Electronic Structure of Undoped and Codoped Molecules . . . . . . . . . . 45

4.5 Transport Properties of Undoped and Codoped Molecular Junctions . . . . 48

4.5.1 Potential Profile . . . . . . . . . . . . . . . . . . 48

4.5 .2 Current-voltage .................... 50

4.5.3 Bias Dependent Transmission ............. 52

4.5.4 Molecule-lead Coupling . . . . . . . . . . . . . . 54

4.5.5 Charge Profile .................. 55

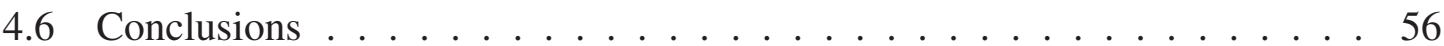




\section{Electronic Structure and Transport Properties in One-dimensional}

Nanowire $\ldots \ldots \ldots \ldots \ldots \ldots \ldots \ldots \ldots$

5.1 Introduction . . . . . . . . . . . . . . . 62

5.2 Quantum Confinement Effect in $\mathrm{PbS}$ Nanowire . . . . . . . . . . . 63

5.2.1 Computational Methods . . . . . . . . . . . . . . 64

5.2.2 Results and Discussions . . . . . . . . . . . . . 66

5.2.3 Strain Induced Phase Transition _ . . . . . . . . . . . . . . . 70

5.3 Gate Field Induced Switching of Current in One Dimensional Nanowire Junction . . . . . . . . . . . . . . . . . 72

5.3.1 Modeling the device . . . . . . . . . . . . . . . . 74

5.3.2 Current-Voltage Characteristics . . . . . . . . . . . . . . 77

5.3.3 Which Density Functional to Choose? . . . . . . . . . . . . . 80

5.3.4 Bias Dependent Transmission . . . . . . . . . . . 83

5.3.5 Orbital Analysis . . . . . . . . . . . . . . . 84

5.4 Conclusions . . . . . . . . . . . . . . . . . . 87

6 Spin Transport in Zero-dimensional Molecular Junction . . . . . . . . . . . 89

6.1 Introduction . . . . . . . . . . . . . . . . 89

6.2 What Determines the Sign Reversal of Tunneling Magnetoresistance? . . . 90

6.3 Computational Details . . . . . . . . . . . . . . . . 94

6.4 Results and Discussions . . . . . . . . . . . . . . . . . . 97

6.4.1 Spin Density \& Energetics . . . . . . . . . . . . . . . 97 
6.4.2 Magnetic Proximity . . . . . . . . . . . . . . . 99

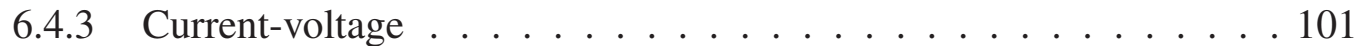

6.4.4 Tunneling Magnetoresistance . . . . . . . . . . . . . . . . . . 102

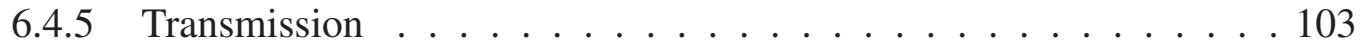

6.4.6 Molecule-lead Coupling . . . . . . . . . . . . . . 104

6.5 Conclusions . . . . . . . . . . . . . . . . . 105

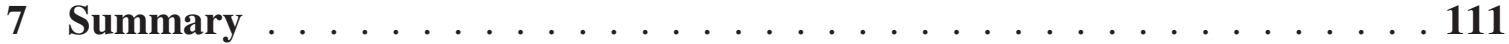

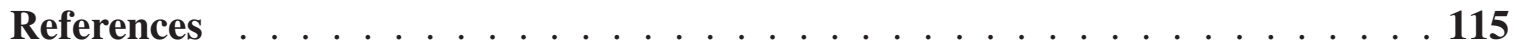

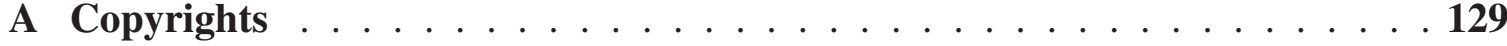

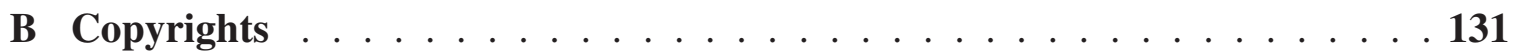

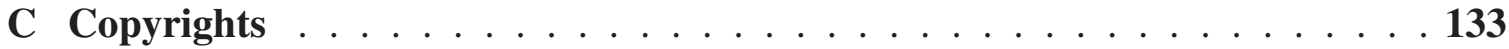




\section{List of Figures}

1.1 Schematic representation of transistor and the evolution of it's channel-length with time. $\ldots \ldots \ldots \ldots \ldots \ldots$

2.1 A schematic representation of an interacting many-electron system. . . . . 6

3.1 A schematic representation of nanoscale junction where a single molecule is sandwiched between two electrodes. . . . . . . . . . . . . . . . . 22

3.2 A schematic representation of a sample (M) sandwiched between two semi-infinite electrodes . . . . . . . . . . . . . . . . . . . . . 29

4.1 Schematic representation of alkali $(\mathrm{X}=\mathrm{Li}, \mathrm{Na}) / \mathrm{B}$-codoped carborane junction. Reprinted figure with permission from Subhasish Mandal and Ranjit Pati, Phys. Rev. B. 83195420 (2011). (C) The American Physical

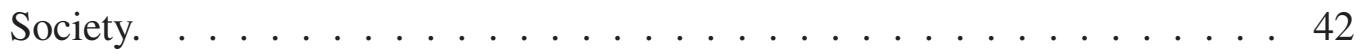

4.2 Energy eigenvalues of $C_{2} B_{10} H_{12}, L i @ C_{1} B_{11} H_{12}$, and $N a @ C_{1} B_{11} H_{12}$. Respective optimized structures are shown in the inset. Reprinted figure with permission from Subhasish Mandal and Ranjit Pati, Phys. Rev. B. 83 195420 (2011). (c) The American Physical Society. . . . . . . . . . . . 45 
4.3 (a) Potential profile of undoped, Li/B-codoped, and $\mathrm{Na/B}$-codoped junctions at $V_{S D} \sim 1 \mathrm{~V}$. (b) Bias dependent potential profile for undoped, (c) Li/B-codoped, and (d) Na/B-codoped junctions. The vertical doted lines depict the location of the atoms along the wire axis in the device. Reprinted figure with permission from Subhasish Mandal and Ranjit Pati, Phys. Rev.

B. 83195420 (2011). (c) The American Physical Society. . . . . . . . . . . 51

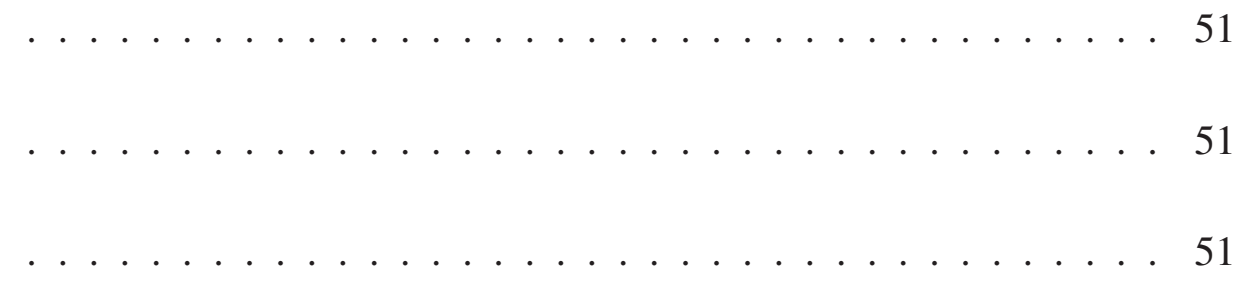

4.4 Realization of single-atom-controlled device through the current-voltage characteristic of undoped, Li/B-codoped, and $\mathrm{Na} / \mathrm{B}$-codoped junctions. Reprinted figure with permission from Subhasish Mandal and Ranjit Pati, Phys. Rev. B. 83195420 (2011). (c) The American Physical Society. . . . . 52

4.5 Bias dependent transmission as a function of injection energy at (a) $\sim 1$ Volt, and (b) $\sim 2$ Volt; Fermi energy is set to zero in the energy scale; dotted lines represent the chemical potential window. Reprinted figure with permission from Subhasish Mandal and Ranjit Pati, Phys. Rev. B. 83195420 (2011). (c) The American Physical Society. . . . . . . . . . 58

$$
\begin{aligned}
& \ldots \ldots \ldots \ldots \ldots \ldots \ldots \ldots \ldots \\
& \ldots \ldots \ldots \ldots \ldots \ldots \ldots \ldots
\end{aligned}
$$


4.6 Charge profile characterizing bias dependent polarization effect on the terminal ' $\mathrm{S}$ ' atoms. Reprinted figure with permission from Subhasish Mandal and Ranjit Pati, Phys. Rev. B. 83195420 (2011). (c) The American Physical Society. . . . . . . . . . . . . . . . . . 59

4.7 Charge profile characterizing bias dependent polarization effect between the alkali atom and the cage $\left(C_{1} B_{11} H_{10} S_{2}\right)$ in (a) Li/B-codoped, and (b) $\mathrm{Na}$ /B-codoped junctions. Reprinted figure with permission from Subhasish Mandal and Ranjit Pati, Phys. Rev. B. 83195420 (2011). (c) The American Physical Society. . . . . . . . . . . . . . . . . . . . 60 60

5.1 Structure of $\mathrm{PbS}$ nanowire of $\mathrm{d} \sim 1.98 \mathrm{~nm}$ : Cross sectional view of one unit cell in (200) direction. S, dark gray (red); Pb, light gray (golden). Reprinted figure with permission from Subhasish Mandal and Ranjit Pati, Chem. Phys. Lett. 479, 244 (2009). (c) The Elsevier. . . . . . . . . . . . . 66

5.2 Electronic energy band structure for bulk $\mathrm{PbS}$ to calibrate our computational approach with experiment. The Fermi level lies at $E=0$. Reprinted figure with permission from Subhasish Mandal and Ranjit Pati, Chem. Phys. Lett. 479, 244 (2009). (c) The Elsevier. . . . . . . . . . . . . 68 
5.3 Electronic energy band structure for $\mathrm{PbS}$ nanowires with different diameters. The Fermi level lies at $E=0$. Reprinted figure with permission from Subhasish Mandal and Ranjit Pati, Chem. Phys. Lett. 479, 244 (2009). (c) The Elsevier. . . . . . . . . . . . . . . . . . . . . 69

5.4 (a) Electronic energy band structures for $\mathrm{PbS}$ nanowire with $\mathrm{d} \sim 1.98 \mathrm{~nm}$ for different $\zeta$ s. The Fermi level lies at E=0. Reprinted figure with permission from Subhasish Mandal and Ranjit Pati, Chem. Phys. Lett. 479, 244 (2009).c The Elsevier. . . . . . . . . . . . . . . . . 71

5.5 The variation of the relative deformation potential energy with $\zeta$. Reprinted figure with permission from Subhasish Mandal and Ranjit Pati, Chem. Phys. Lett. 479, 244 (2009). (c) The Elsevier. . . . . . . . . . . . . . . . . 73

5.6 Schematic representation of a $\mathrm{PbS}$ nanowire junction; solid arrows show the direction of the applied gate field. Reprinted figure with permission from Subhasish Mandal and Ranjit Pati, Phys. Rev. B. 84115306 (2011). (c) The American Physical Society. . . . . . . . . . . . . . . 75

5.7 Electrostatic potential profile of the NW junction in the absence of gate bias for two different $V_{s d}$. Reprinted figure with permission from Subhasish Mandal and Ranjit Pati, Phys. Rev. B. 84115306 (2011). (c) The American Physical Society . . . . . . . . . . . . . . . . 78 
5.8 Current-Voltage characteristics with different $V_{g}$ for $\mathrm{PbS}$ nanowire junctions. The insets show $I_{s d}-V_{g}$ plot for different $V_{s d}$. Reprinted figure with permission from Subhasish Mandal and Ranjit Pati, Phys. Rev. B. 84 115306 (2011). (c) The American Physical Society . . . . . . . . . . . 79

5.9 Current-Voltage characteristics with different $V_{g}$ for $\mathrm{PbSe}$ nanowire junctions. The insets show $I_{s d^{-}} V_{g}$ plot for different $V_{s d}$. Reprinted figure with permission from Subhasish Mandal and Ranjit Pati, Phys. Rev. B. 84 115306 (2011). (c) The American Physical Society . . . . . . . . . . 80

5.10 Current-Voltage characteristics of PbSNW for different exchange-correlation functionals at $V_{g}=0 \mathrm{~V}$. Reprinted figure with permission from Subhasish Mandal and Ranjit Pati, Phys. Rev. B. 84 115306 (2011). (c) The American Physical Society. . . . . . . . . . . 82

5.11 Bias dependent transmission function as a function of injection energy for different gate bias at $V_{s d} \sim 0.6 \mathrm{~V}$. The Fermi energy is set to zero in the energy scale; dotted lines represent the chemical potential window. Notation: L0, L1, L2, L3, and L4 refer to LUMO, LUMO+1, LUMO+2, LUMO+3, and LUMO+4. Reprinted figure with permission from Subhasish Mandal and Ranjit Pati, Phys. Rev. B. 84115306 (2011). (c) The American Physical Society. . . . . . . . . . . . . . . . . 84 
5.12 Stark shift as a function of gate bias. Notation: L0, L1, L2, L3, and L4 refer to LUMO, LUMO+1, LUMO+2, LUMO+3, and LUMO+4. A fixed $V_{s d}$ of $\sim 0.6 \mathrm{~V}$ is used. Reprinted figure with permission from Subhasish Mandal and Ranjit Pati, Phys. Rev. B. 84115306 (2011). (c) The American Physical Society. . . . . . . . . . . . . . . 85

5.13 Schematic representation of orbital controlled mechanism for the PbSNW FET. In the ON state $\left(V_{g}>V_{g}^{t h}\right)$, orbital mixing produces $p$-component at the $\mathrm{S}$ atoms resulting in an inter-layer orbital interaction along the channel (z) axis. The S-atom has only $s$-component in the OFF state $\left(V_{g}<V_{g}^{t h}\right)$. Reprinted figure with permission from Subhasish Mandal and Ranjit Pati, Phys. Rev. B. 84115306 (2011). (c) The American Physical Society. . . . 86

6.1 Electron spin density plot for (a) parallel, and (b) anti-parallel alignment of spins at the two electrodes. Red represents positive (up) spin density and blue represents negative (down) spin density. Solid arrow represents the direction of magnetization at the electrodes. Reprinted figure with permission from Subhasish Mandal and Ranjit Pati, ACS Nano 6, 3580 (2012). (c) The American Chemical Society. . . . . . . . . . . . . 93

6.2 Bias dependent exchange energy $\left(E_{P C}-E_{A P C}\right)$ for the spin-valve device with three interfacial distances $(d)$. Reprinted figure with permission from Subhasish Mandal and Ranjit Pati, ACS Nano 6, 3580 (2012). (c) The American Chemical Society. . . . . . . . . . . . . . . . 95 
6.3 Schematic representation of spin-profile along the wire axis for both the parallel and anti-parallel configurations; up(down) arrows refer to the positive (negative) magnetic moment. Reprinted figure with permission from Subhasish Mandal and Ranjit Pati, ACS Nano 6, 3580 (2012). (c) The American Chemical Society. . . . . . . . . . . . . . . . . . 97

6.4 Bias dependent magnetic moment of the molecular spacer for (a) parallel, and (b) anti-parallel alignment of spins at two electrodes. $d$ refers to the interfacial distance. Reprinted figure with permission from Subhasish Mandal and Ranjit Pati, ACS Nano 6, 3580 (2012). (c) The American Chemical Society. . . . . . . . . . . . . . . . . 106

6.5 Bias dependent molecular orbital for HOMO; panel in green shows molecular orbital for spin up states while panel in red shows for spin down states. Reprinted figure with permission from Subhasish Mandal and Ranjit Pati, ACS Nano 6, 3580 (2012). (c) The American Chemical Society. . . . . 107

6.6 Current-voltage characteristics for the parallel and anti-parallel configurations with $d=$ (a) 2.06, (b) 2.12, and (c) $2.00 \AA$ A. Reprinted figure with permission from Subhasish Mandal and Ranjit Pati, ACS Nano 6, 3580 (2012). (c) The American Chemical Society. . . . . . . . . . . . . . 108 
(b)

(c)

6.7 Bias dependent tunnel magnetoresistance for three interfacial distances $(d)$.

Reprinted figure with permission from Subhasish Mandal and Ranjit Pati, ACS Nano 6, 3580 (2012). (c) The American Chemical Society. . . . . . . 109

6.8 Bias dependent transmission function for $d=$ (a) 2.06 and (b) $2.00 \AA$. The Fermi level lies at $E=0$. The dotted lines show the chemical potential window. $H_{0}, H_{1}, H_{2}, H_{3}, H_{4}, H_{5}, H_{6}, H_{7}, H_{8}$ represent HOMO, HOMO-1, HOMO-2, HOMO-3, HOMO-4 HOMO-5, HOMO-6, HOMO-7, and HOMO-8, respectively. $L_{0}$ and $L_{1}$ represent LUMO and LUMO+1, respectively. Reprinted figure with permission from Subhasish Mandal and Ranjit Pati, ACS Nano 6, 3580 (2012). (c) The American Chemical Society. 110

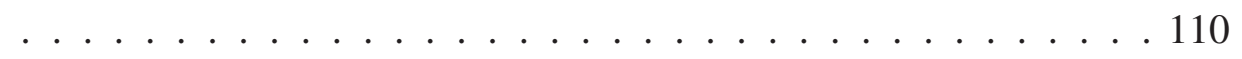

A.1 Copyright permission from The Elsevier for the articles by Subhasish Mandal and Ranjit Pati, Chem. Phys. Lett. 479, 244 (2009). . . . . . . . . 130

B.1 The copyright permission from The American Physical Society for the articles by Subhasish Mandal and Ranjit Pati, Phys. Rev. B. 83, 195420 (2011) and Phys. Rev. B. 84115306 (2011). . . . . . . . . . . . . . 132 
C.1 Copyright permission from The American Chemical Society for the articles

by Subhasish Mandal and Ranjit Pati, ACS Nano 6, 3580 (2012). . . . 134 


\section{List of Tables}

4.1 Calculated $\Delta_{F E}$ (eV/atom), bond lengths in undoped, Li/B-codoped, and $\mathrm{Na}$ /B-codoped carborane molecules. . . . . . . . . . . . . . . 47

5.1 Calculated diameter (d), average $\mathrm{Pb}-\mathrm{S}$ bond length $(l), E_{c}$, and $\Delta E_{g}$ for $\mathrm{PbS}$

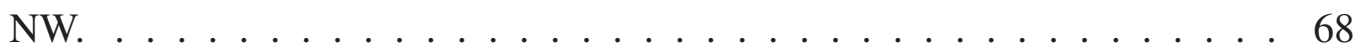





\section{Acknowledgments}

It's my pleasure to thank many people without whom this thesis would not have been possible. First, I would like to express my deep and sincere gratitude to my $\mathrm{PhD}$ advisor Professor Ranjit Pati for his enormous support and excellent guidance. From the very first day when I joined his group until the day I finished my work, he was equally patient and extremely enthusiastic towards teaching and guiding me. No word is sufficient to express my gratitude for his excellent guidance in my academic and personal issues throughout my stay at Michigan Tech.

I am grateful to Professor Ravindra Pandey for his support, input to this thesis, and constant encouragement as a committee member and as the chair of the Department of Physics. I would like to thank my doctoral committee members, Professor Maximilian

Seel, Professor Gregory Odegard, Professor Rabindra Pandey, and Professor Ranjit Pati for their valuable suggestions and their critical review on this thesis.

I would like to thank all the members of Prof. Pati's research group- Pavan, Puspamitra, Partha, and Kamal. They are the people with whom I have discussed, learned, and spent some great times. My thanks also goes to Mike and Brandon. I would like to acknowledge IT-help for the computing facilities. In particular, to Gowtham for taking so much care for 
the cluster. I am also thankful to him for the help in $\mathrm{IAT}_{\mathrm{E}} \mathrm{X}$ template to write this thesis.

I would like to thank Prof. Samuel Trickey from the Dept. of Physics and Chemistry at the University of Florida for his helpful insights and discussions in two of my major projects. I would also like to thank him for his series of lectures in Density Functional Theory that he delivered here at Michigan Tech. Throughout my journey, a lot of theoretical physicists at IIT Madras have inspired me to pursue my career in Physics. In this context, the person who has inspired me the most in my masters studies is Prof. Bhanu Pratap Das with whom I did a summer internship at the Indian Institute of Astrophysics and decided to pursue research in the theoretical and computational physics. I would like to thank him in this regard for his encouragements.

In the course of studying Physics at Michigan Tech, I met a lot of people who were very open to discuss Physics with me. My first thanks goes to Biju for his encouragement and guidance. He was my special Physics friend with whom I was engaged in long night Physics discussions. Indeed, I enjoyed those times. I would like to thank Saikat, Wil Slough, Madhusudan, and Douglas for all the discussions we made. Physicists drink coffee. The new coffee shop at the library is a place where I meet a lot of people everyday. I would like to thank Niloy and Niraj for drinking coffee with me and taking part in all Physics and non-Physics conversations.

I would like to thank the department coordinator Andy and department secretaries Marg and Kathy for maintaining everything smoothly in the department. 
I am grateful to the graduate school at Michigan Tech for offering me the finishing fellowship for the summer-2012. I am also thankful to the Dept. of Physics for offering me the Graduate Teaching Assistantship for the first two semesters of my graduate studies. My acknowledgment also goes to the National Science Foundation (NSF) of the United States for the financial support throughout the program.

Lastly and most importantly, I would like to thank my parents, my elder brother and brother-in-law, my maternal uncle and aunts back in India for their love and constant mental support. I dedicate this thesis to them. 



\section{Abstract}

Recently nanoscale junctions consisting of 0-D nanostructures (single molecule) or 1-D nanostructures (semiconducting nanowire) sandwiched between two metal electrodes are successfully fabricated and characterized. What lacks in the recent developments is the understanding of the mechanism behind the observed phenomena at the level of atoms and electrons. For example, the origin of observed switching effect in a semiconducting nanowire due to the influence of an external gate bias is not yet understood at the electronic structure level. On the same context, different experimental groups have reported different signs in tunneling magneto-resistance for the same organic spin valve structure, which has baffled researchers working in this field. In this thesis, we present the answers to some of these subtle questions by investigating the charge and spin transport in different nanoscale junctions. A parameter-free, single particle Green's function approach in conjunction with a posteriori density functional theory (DFT) involving a hybrid orbital dependent functional is used to calculate the tunneling current in the coherent transport limit. The effect of spin polarization is explicitly incorporated to investigate spin transport in a nanoscale junction. Through the electron transport studies in $\mathrm{PbS}$ nanowire junction, a new orbital controlled mechanism behind the switching of the current is proposed. It can explain the switching behavior, not only in $\mathrm{PbS}$ nanowire, but in other lead-chalcogenide nanowires as well. Beside this, the electronic structure properties of this nanowire are studied using periodic DFT. The quantum confinement effect was investigated by calculating the bandgap of $\mathrm{PbS}$ 
nanowires with different diameters. Subsequently, we explain an observed semiconducting to metallic phase transition of this nanowire by calculating the bandgap of the nanowire under uniform radial strain. The compressive radial strain on the nanowire was found to be responsible for the metallic to semiconducting phase transition.

Apart from studying one dimensional nanostructure, we also present transport properties in zero dimensional single molecular junctions. We proposed a new codoping approach in a single molecular carborane junction, where a cation and an anion are simultaneously doped to find the role of a single atom in the device. The main purpose was to build a molecular junction where a single atom can dictate the flow of electrons in a circuit. Recent observations of both positive and negative sign in tunneling magnetoresistance (TMR) the using same organic spin-valve structure has mystified researchers. From our spin dependent transport studies in a prototypical organic molecular tunneling device, we found that a $3 \%$ change in metal-molecule interfacial distance can alter the sign of TMR. Changing the interfacial distance by $3 \%$, the number of participating eigenstates as well as their orbital characteristic changes for anti-parallel configuration of the magnetization at the two electrodes, leading to the sign reversal of the TMR. Apart from this, the magnetic proximity effect under applied bias is investigated quantitatively, which can be used to understand the observed unexpected magnetism in carbon based materials when they are in close proximity with magnetic substrates. 


\section{Chapter 1}

\section{Introduction}

Nanotechnology is the technology that manipulates matters at the scale of atoms and molecules. As Feynman says, "there's plenty of room at the bottom," nanotechnology can substantially offer the scope to understand matter at the scale of atoms and molecules. While classical physics fails to explain at this scale, quantum mechanics, in its most arcane and practical guises, is used to understand the world of nano or sub-nano particles. Researchers have harnessed this abstract quantum theory in the nanoworld by building devices out of these nanometer-sized particles which are used in our daily life. Especially in the past three decades silicon-based technology has revolutionized our world by offering us ultra-thin integrated circuits built on a single chip. They are now used in almost all electronic devices starting from our cars to the mobile phones. The thirst to build a smaller and faster device is still not satisfied. 

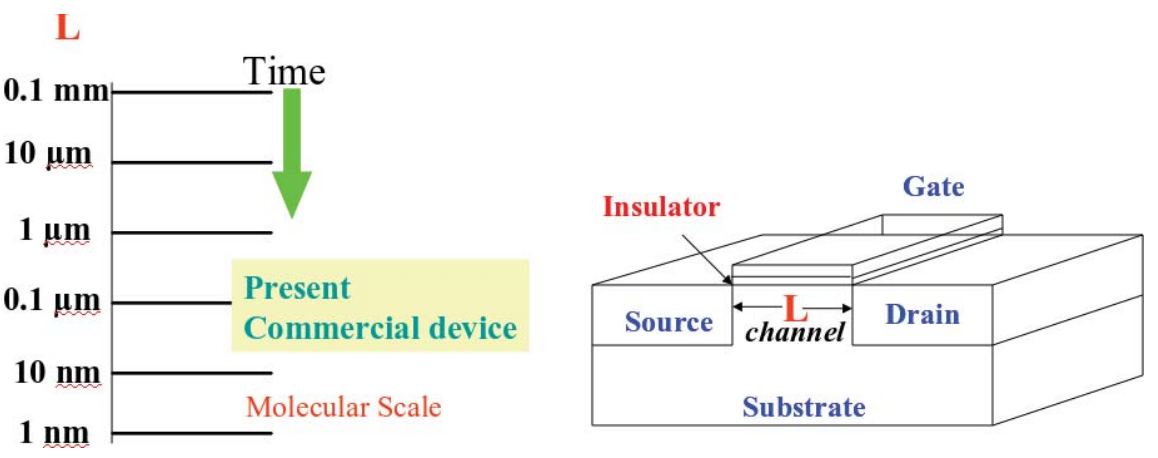

Figure 1.1: Schematic representation of transistor and the evolution of it's channel-length with time.

The main 'working unit' in a computer device is a transistor, which is schematically shown in Fig. 1.1. Now, to "pack" more and more transistors in a device in order to make it faster and smaller, one needs to reduce down the width of the insulating layer (typically made of $\mathrm{SiO}_{2}$ ). According to Moore's law, in the year 2020 the insulating layer would be as thick as five atoms [1]. Reducing further would be impossible as electrons in the gate electrode can directly penetrate into the channel and destroy the transistor. Thus, the road for the silicon industry is predicted to reach its end by the year of 2020 due to the problem which is technically known as "quantum leakage". As the size of the conventional silicon based field effect transistor is inching toward its fundamental limit of miniaturization, new quantum controlled, nanoscale materials have emerged as one of the promising options to meet this physical challenges.

Fig. 1.1 shows how the length of the channel which connects the source and drain in a transistor, started becoming shorter and shorter as time progresses. In present commercial 
computer device which has about billion of transistors has channel length of about few hundreds of nanometers. We can easily realize that reducing down of the channel length further, one can reach to the nanoscale regime where the channel can be build out of a single molecule or a nanowire.

If we look carefully in the history of science, we have seen each new major discovery permits tool for much more discoveries in the future. This process goes on. The envision of Aviram and Ratner to build a single molecular device was one of such major inspirations [2]. The realization of that envision by successfully connecting a molecule in between two metal electrodes and subsequently measuring the tunneling current has opened up a new horizon in nanoscience research $[3,4,5,6]$. Several groups have reported conduction, rectification, and switching phenomena $[4,7,8,9]$ in nanoscale junctions. These devices are mainly made of zero-dimensional single molecules and one-dimensional semiconducting nanowires or nanotube. These atomic scale devices exhibit interesting features like negative differential resistance [8], phase transition [10], spin valve effect [9]. Understanding these issues by modeling at the scale of atom and electron is a challenging task. The first challenge is to model this open device without proper knowledge of the contact geometry. The first principles approach to the transport problem will not only help understanding the phenomena at the level of electrons and atoms, but at the same time it will help the experimentalist to build the future electronic devices. Here, using density functional theory we try to answer some important questions associated with recent observations in different transport measurements in single molecular and nanowire 
junctions. We also describe a new approach towards achieving the ultimate limit of miniaturization where a single atom can dictate the flow of the electrons in a circuit. The rest of the thesis is organized as follows. Density functional theory is described in Chapter 2 followed by theory of quantum transport in nanoscale systems in Chapter 3. Results are described in subsequent chapters. This thesis ends with a brief summary in Chapter 7. 


\section{Chapter 2}

\section{Density Functional Theory}

\subsection{Introduction}

It is impossible to obtain an exact solution of a many-body problem. To gain an accurate description of interacting quantum many-body problem has been a challenge from a long time. Particularly when the systems consists of few atoms, the computational cost for the accurate results involving the traditional wavefunction approach goes up dramatically. Density functional theory (DFT) maintains an important balance between the accuracy and the computational cost [11]. In recent years, DFT has gained a huge attention in the field of physics, chemistry and biology [12]. It is a first principle approach where the computations are done without assuming any parameter that describes the bonding, the shape of the 
charge and density or the potential [13]. DFT solves an interacting problem by mapping into a much simpler non-interacting problem. In this chapter, I describe some of the basic ideas behind density functional calculations.

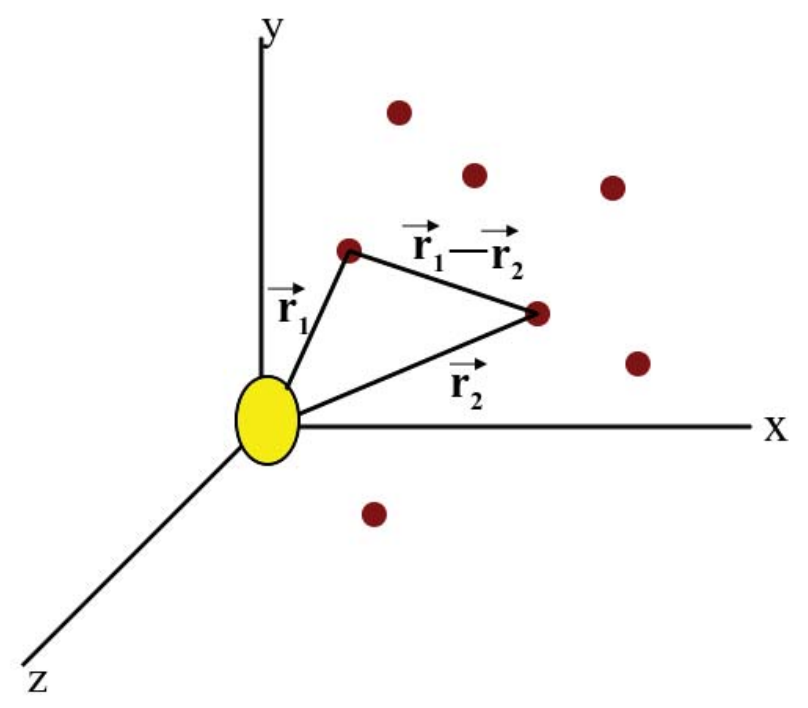

Figure 2.1: A schematic representation of an interacting many-electron system.

\subsection{Many Electron Hamiltonian}

Let me first write down the many-body Hamiltonian for an electronic system $[14,15]$ :

$$
\hat{H}=\frac{1}{2} \sum_{i}^{N_{e}} \nabla_{i}^{2}-\sum_{i}^{N_{e}} \sum_{I}^{N_{n}} \frac{Z_{I}}{\left|\vec{r}_{i}-\vec{R}_{I}\right|}+\frac{1}{2} \sum_{i}^{N_{e}} \sum_{j \neq i}^{N_{e}} \frac{1}{\left|\vec{r}_{i}-\vec{r}_{j}\right|}+\frac{1}{2} \sum_{I}^{N_{n}} \sum_{J \neq I}^{N_{n}} \frac{Z_{I} Z_{J}}{\left|\vec{R}_{I}-\vec{R}_{J}\right|}+\frac{1}{2 M_{I}} \sum_{I}^{N_{n}} \nabla_{I}^{2}
$$


In the above equation $e=\hbar=m_{e}=1 . \vec{r}_{i}, \vec{R}_{I}$ are the coordinate of the electrons and nuclei. $N_{e}$ and $N_{n}$ are the number of electrons and nuclei present in the system; $M_{I}$ represents the mass of the I-th nucleus. First, we make the Born-Oppenheimer approximation [16, 14] in which we neglect the last two terms in the above equation that describes the relative motion of heavy nuclei. We treat the nuclei as fixed points. If you look carefully in the above Hamiltonian, the first and the second terms depends only on single electron coordinate and therefore, it is called as a single-electron term. However, the third term describing the electron-electron interaction depends on the pair of electrons. So the above Hamiltonian can be written as:

$$
H=\frac{1}{2} \sum_{i}^{N_{e}} \nabla_{i}^{2}-\sum_{i}^{N_{e}} \sum_{I}^{N_{n}} \frac{Z_{I}}{\left|\vec{r}_{i}-\vec{R}_{I}\right|}+\frac{1}{2} \sum_{i}^{N_{e}} \sum_{j \neq i}^{N_{e}} \frac{1}{\left|\vec{r}_{i}-\vec{r}_{j}\right|}
$$

or,

$$
H=\sum_{i} \hat{h_{1}}\left(\vec{x}_{i}\right)+\frac{1}{2} \sum_{i \neq j} \hat{h_{2}}\left(\vec{x}_{i}, \vec{x}_{j}\right)
$$




\subsection{Hatree-Fock Method}

In Hartee-Fock method the non-interacting electron wavefunction is expressed as a Slater determinant as follows [17]:

$$
\Phi\left(\overrightarrow{x_{1}}, \overrightarrow{x_{2}}, . . \overrightarrow{x_{n}}\right)=\frac{1}{\sqrt{N}}\left|\begin{array}{cccc}
\phi_{1}\left(\overrightarrow{x_{1}}\right) & \phi_{2}\left(\overrightarrow{x_{1}}\right) & \ldots & \phi_{3}\left(\overrightarrow{x_{1}}\right) \\
\phi_{1}\left(\overrightarrow{x_{2}}\right) & \phi_{2}\left(\overrightarrow{x_{2}}\right) & \ldots & \phi_{3}\left(\overrightarrow{x_{2}}\right) \\
\cdot & . & & \cdot \\
\cdot & . & & \cdot \\
\phi_{1}\left(\overrightarrow{x_{N}}\right) & \phi_{2}\left(\overrightarrow{x_{N}}\right) & \ldots & \phi_{3}\left(\overrightarrow{x_{N}}\right)
\end{array}\right|
$$

Although Hatree-Fock approximation is a many-body technique, it is based on a single-particle picture where the electrons are considered as occupying single-particle orbitals $\left(\phi_{i}\left(\vec{x}_{i}\right)\right)$. Electron sees the other electrons through an effective potential. Now, the next task is to write the Schrodinger equation in terms of the orbitals. First, it can be easily shown that $[17,18,19]$ :

$$
\left\langle\Phi\left|\sum_{i} \hat{h}_{1}\left(\vec{x}_{n}\right)\right| \Phi\right\rangle=\sum_{i}^{N_{e}}\left\langle\phi_{i}\left|\hat{h}_{1}\right| \phi_{i}\right\rangle
$$


and,

$$
\left\langle\Phi\left|\frac{1}{2} \sum_{i \neq j} \hat{h}_{2}\left(\vec{x}_{i}, \vec{x}_{j}\right)\right| \Phi\right\rangle=\frac{1}{2} \sum_{i, j}^{N_{e}}\left(\left\langle\phi_{i} \phi_{j}\left|\hat{h}_{2}\right| \phi_{1} \phi_{j}\right\rangle-\left\langle\phi_{j} \phi_{i}\left|\hat{h}_{2}\right| \phi_{1} \phi_{j}\right\rangle\right)
$$

So, we get,

$$
\begin{array}{r}
\left\langle\Phi\left|\sum_{i} \hat{H}\left(\vec{x}_{i}\right)\right| \Phi\right\rangle=\sum_{i}^{N_{e}}\left\langle\phi_{i}\left|\hat{h}_{1}\right| \phi_{i}\right\rangle+\frac{1}{2} \sum_{i, j}^{N_{e}}\left(\left\langle\phi_{i} \phi_{j}\left|\hat{h}_{2}\right| \phi_{1} \phi_{j}\right\rangle-\left\langle\phi_{j} \phi_{i}\left|\hat{h}_{2}\right| \phi_{i} \phi_{j}\right\rangle\right) \\
=\sum_{i} h_{i}+\frac{1}{2} \sum_{i, j}\left(J_{i j}-K_{i j}\right)
\end{array}
$$

where $h_{i}=\int \phi_{i}^{*}(\vec{x})\left[-\frac{1}{2} \nabla^{2}+v(\vec{x})\right] \phi_{i}(\vec{x}) d \vec{x}$. In the eq. 2.7, the first and second terms are the single-body operator while the third term is the integral operator. $J_{i j}=$ $\iint \phi_{i}\left(\overrightarrow{x_{1}}\right) \phi_{i}^{*}\left(\overrightarrow{x_{1}}\right) \frac{1}{\left|\overrightarrow{r_{i}}-\overrightarrow{r_{j}}\right|} \phi_{j}\left(\overrightarrow{x_{2}}\right) \phi_{j}^{*}\left(\overrightarrow{x_{2}}\right) d \overrightarrow{x_{1}} d \overrightarrow{x_{2}}$ is the coulomb integrals, which describes the classical interaction of electron distribution. This is referred to the direct term while $K_{i j}=$ $\iint \phi_{i}^{*}\left(\vec{x}_{1}\right) \phi_{j}\left(\vec{x}_{1}\right) \frac{1}{\mid \overrightarrow{r_{i}-\vec{r}_{j} \mid}} \phi_{i}\left(\overrightarrow{x_{2}}\right) \phi_{j}^{*}\left(\overrightarrow{x_{2}}\right) d \overrightarrow{x_{1}} d \overrightarrow{x_{2}}$ is called as the exchange term. The exchange term has no classical analogue and is a direct consequence of the antisymmetric property of the wavefunction.

Now we will apply the variational principle. The expectation value of energy has to be minimized with respect to the coefficient of the basis function with the constraint given by 
the normalization condition. So the condition to be satisfied is then:

$$
\delta\left[\left\langle\Phi\left|\sum_{i} \hat{H}\left(\vec{x}_{n}\right)\right| \Phi\right\rangle-\sum_{i, j} \lambda_{i j}\left(\left\langle\phi_{i} \mid \phi_{j}\right\rangle-\delta_{i j}\right)\right]=0
$$

where $\lambda_{i j}$ s are the Lagrange multipliers. Now we take the derivatives in eq. 2.7. It is customary to write in the following form $[18,19]$ :

$$
\left[\hat{h}_{1}+\sum_{i}\left(\hat{J}_{i}-\hat{K}_{i}\right)\right] \phi_{k}=\sum_{i} \lambda_{k i} \phi_{i}
$$

\subsection{Energy in Terms of Density}

As described above, the Hatree-Fock theory works with N-electron wavefunction $\Phi\left(\overrightarrow{x_{1}}, \overrightarrow{x_{2}} \ldots ., \overrightarrow{x_{N}}\right)$, which is a function of $3 \mathrm{~N}$ degrees of freedom. This becomes computationally very expensive and extremely difficult if the system consists of a bundles of atoms. A very smart way to overcome this issue is to express the Hamiltonian through electron density [17, 18, 12] $\left[n(\vec{r})=\langle\Phi|\hat{n}(\vec{r})| \Phi\rangle=N \int\left|\Phi\left(\vec{r}, \ldots, \vec{r}_{N}\right)\right|^{2} d \vec{r}_{2} \ldots d \vec{r}_{N}\right.$, with $\int d \vec{r} n(\vec{r})=N$ Rewriting the Hamiltonian once again:

$$
H=\frac{1}{2} \sum_{i}^{N_{e}} \nabla_{i}^{2}-\sum_{i}^{N_{e}} \sum_{I}^{N_{n}} \frac{Z_{I}}{\left|\vec{r}_{i}-\vec{R}_{I}\right|}+\frac{1}{2} \sum_{i}^{N_{e}} \sum_{j \neq i}^{N_{e}} \frac{1}{\left|\vec{r}_{i}-\vec{r}_{j}\right|}=\hat{T}+\hat{V}_{n e}+\hat{V}_{e e}
$$


Now, we can write the nuclear-electron interaction energy in terms of the density in the following way [18]:

$$
\begin{array}{r}
E_{n e}=\left\langle\Phi\left(\vec{r}_{1}, \ldots, \vec{r}_{N}\right)\left|\hat{V}_{n e}\right| \Phi\left(\vec{r}_{1}, \ldots, \vec{r}_{N}\right)\right\rangle \\
=-\frac{1}{N_{e}} \sum_{I}^{N_{n}}\left[\int \frac{Z_{I} d \vec{r}_{1} n\left(\vec{r}_{1}\right)}{\left|\vec{r}_{1}-\vec{R}_{I}\right|}+\int \frac{Z_{I} d \vec{r}_{2} n\left(\vec{r}_{2}\right)}{\left|\vec{r}_{2}-\vec{R}_{I}\right|}+\ldots\right] \\
=-\sum_{I}^{N_{n}} \int \frac{Z_{I} d \vec{r} n(\vec{r})}{\left|\vec{r}-\vec{R}_{I}\right|}=\int n(\vec{r}) V_{n e}(\vec{r}) d \vec{r}
\end{array}
$$

However, the equivalent derivations for the electron-electron interaction term is not so simple. Instead, it is expressed in terms of two-particle density like the following [18]:

$$
E_{e e}=\frac{1}{2} \iint d \vec{r} d \vec{r}^{\prime} \frac{n^{(2)}\left(\vec{r}, \vec{r}^{\prime}\right)}{\left|\vec{r}-\vec{r}^{\prime}\right|}
$$

where $n^{(2)}$ can be interpreted as the probability of an electron to exist at a point $\vec{r}$, given that a second electron exist at $\vec{r}^{\prime}$. However, if the electrons are completely uncorrelated and there exists only one-particle density, then $n^{(2)}$ can be expressed as $[17,18]$ :

$$
n^{(2)}=n(\vec{r}) n(\vec{r})+\Delta n^{(2)}(\vec{r}, \vec{r})
$$

So the electron-electron term, which makes the many-body problem so difficult, can now 
be expressed as:

$$
E_{e e}=\frac{1}{2} \iint d \vec{r} d \vec{r}^{\prime} \frac{n(\vec{r}) n\left(\vec{r}^{\prime}\right)}{\left|\vec{r}-\vec{r}^{\prime}\right|}+\Delta E_{e e}
$$

The second term is due to the correction term in eq. 2.15. Now writing the kinetic energy in terms of single-particle density is even more difficult as it involves the derivative operator. Before I describe them, I would like to discuss two important theorems given by Hohenberg and Kohn in 1964.

\subsection{The Hohenberg-Kohn Theorem}

Theorem I: For any system of interacting particles in an external potential $V_{\text {ext }}(\vec{r})$, the density is uniquely determined or the external potential $V_{\text {ext }}(\vec{r})$ is determined, within a trivial additive constant, by the electron density. [20]

Proof: The proof is by contradiction. Suppose there exist two potentials $V_{\text {ext } 1}(\vec{r})$ and $V_{\text {ext2 }}(\vec{r})$, which differ by more than a constant but yield the same density. There would have to be two ground-state wavefunctions $\Phi_{1}$ and $\Phi_{2}$, and they would belong to two distinct Hamiltonians $H^{1}$ and $H^{2}$ respectively. Let's assume that the ground state is non-degenerate. So, by variational principle, no wavefunction would give an energy less than the expectation 
value of $H^{1}$ with $\Phi^{1}$ [17]. Thus,

$$
\left\langle\Phi^{1}\left|H^{1}\right| \Phi^{1}\right\rangle<\left\langle\Phi^{2}\left|H^{1}\right| \Phi^{2}\right\rangle
$$

As we have identical ground state densities for two Hamiltonians, we can write

$$
\left\langle\Phi^{2}\left|H^{1}\right| \Phi^{2}\right\rangle=\left\langle\Phi^{2}\left|H^{2}\right| \Phi^{2}\right\rangle+\int n_{0}(\vec{r}) d \vec{r}\left[V_{\text {ext } 1}(\vec{r})-V_{\text {ext } 2}(\vec{r})\right]
$$

Similarly,

$$
\left\langle\Phi^{1}\left|H^{2}\right| \Phi^{1}\right\rangle=\left\langle\Phi^{1}\left|H^{1}\right| \Phi^{1}\right\rangle+\int n_{0}(\vec{r}) d \vec{r}\left[V_{\text {ext } 2}(\vec{r})-V_{\text {ext } 2}(\vec{r})\right]
$$

Adding the above two equations,

$$
E^{1}+E^{2}<E^{2}+E^{1}
$$

This clearly is a contradiction and hence, the ground-state density uniquely determines the external potential up to a constant.

Theorem II: The ground state energy can be obtained variationally; the density that minimizes the total energy is the exact ground state density. 
From the above theorem, we understand that the external potential is uniquely determined by the density, and the potential uniquely determines the ground-state wavefunction. One may write the energy in terms of the density functional. As we have already seen (except the kinetic energy term, which I will describe in the next section), we can convert the total energy expression from the wavefunction representation to the density representation.

$$
E[n]=T[n(\vec{r})]+V_{n e}[n(\vec{r})]+V_{e e}[n(\vec{r})]=\int V_{e x t}(\vec{r}) n(\vec{r}) d \vec{r}+F[n(\vec{r})]
$$

where $F[n(\vec{r})]$ is a universal functional because the treatment of the kinetic and the internal potential energies are the same for all systems. Now, in the ground state, the energy can be determined by the unique ground-state density, $n^{1}(\vec{r})$,

$$
E^{1}=E\left[n^{1}\right]=\left\langle\Phi^{1}\left|H^{1}\right| \Phi^{1}\right\rangle
$$

We know from the variational principle, a different density, $n^{2}(\vec{r})$ must give a higher energy.

$$
E^{1}=E\left[n^{1}\right]=\left\langle\Phi^{1}\left|H^{1}\right| \Phi^{1}\right\rangle\left\langle\left\langle\Phi^{2}\left|H^{1}\right| \Phi^{2}\right\rangle=E^{2}\right.
$$

So we can say that by minimizing the total energy with respect to $n(\vec{r})$, we can obtain the total energy of the ground state. The correct density that minimizes the energy is then the ground state density. 


\subsection{Kohn-Sham Method}

We have noted before that writing the kinetic energy in terms of the functional is a difficult task. Kohn and Sham in 1965, found a brilliant approach that turned the density functional theory into a practical tool for rigorous calculations [21, 22]. The method is known as the Kohn-Sham method. Here we assume that the density can be written as the sum of the norm square of a collection of single particle orbitals:

$$
n(\vec{r})=\sum_{n}^{N_{e}}\left|\phi_{n}(\vec{r})\right|^{2}
$$

These orbitals are called Kohn-Sham orbitals. We express the kinetic energy as a single particle kinetic energy plus a correction term.

$$
T=-\frac{1}{2} \sum_{n}^{N_{e}} \int d \vec{r} \phi_{n}^{*} \nabla^{2} \phi_{n}(\vec{r})+\Delta T
$$

Finally, the total energy can be written as:

$$
E=-\frac{1}{2} \sum_{n}^{N_{e}} \int d \vec{r} \phi_{n}^{*} \nabla^{2} \phi_{n}(\vec{r})+\Delta T+\int n(\vec{r}) V_{n e}(\vec{r}) d \vec{r}+\frac{1}{2} \iint d \vec{r} d \vec{r}^{\prime} \frac{n(\vec{r}) n\left(\vec{r}^{\prime}\right)}{\left|\vec{r}-\vec{r}^{\prime}\right|}+\Delta E_{e e}
$$

In order to write the correction terms in terms of density, we define exchange-correlation 
energy as:

$$
E_{x c}=\Delta E_{e e}+\Delta T
$$

Where

$$
E_{x c}=\int n(\vec{r}) \varepsilon_{x c}(n) d \vec{r}
$$

In the above equation, $\varepsilon_{x c}(n)$ is a simple function of $\mathrm{n}$. Thus, the total energy can be written as:

$$
E=-\frac{1}{2} \sum_{n}^{N_{e}} \int \phi_{n}^{*} \nabla^{2} \phi_{n}(\vec{r}) d \vec{r}+\int n(\vec{r}) V_{n e}(\vec{r}) d \vec{r}+\frac{1}{2} \iint d \vec{r}^{\prime} \frac{n(\vec{r}) n\left(\vec{r}^{\prime}\right)}{\left|\vec{r}-\vec{r}^{\prime}\right|} d \vec{r}+\int n(\vec{r}) \varepsilon_{x c}(n) d \vec{r}
$$

The next step is straight forward. We minimize the total energy with respect to the orbitals to get the orbitals that produce the ground state energy. Now minimizing the energy in eq. 2.30 with respect to $\phi_{i}^{*}$ (instead of $\left.\phi_{i}\right)$, we get

$$
\begin{aligned}
-\frac{1}{2} \nabla^{2} \phi_{n}(\vec{r})+\left[V_{\text {ext }}(\vec{r})+\int \frac{n\left(\vec{r}^{\prime}\right)}{\left|\vec{r}-\vec{r}^{\prime}\right|} d \vec{r}^{\prime}+\varepsilon_{x c}[n]+n(\vec{r}) \frac{\delta \varepsilon_{x c}[n]}{\delta n(\vec{r})}\right] \phi_{i}(\vec{r}) & =\varepsilon_{i} \phi_{i}(\vec{r})(2.3 \\
\Rightarrow-\frac{1}{2} \nabla^{2} \phi_{n}(\vec{r})+\left[V_{\text {ext }}(\vec{r})+V_{H}(\vec{r})+V_{x c}(\vec{r})\right] \phi_{i}(\vec{r}) & =\varepsilon_{i} \phi_{i}(\vec{r})(2 . \\
\Rightarrow\left[T+V_{\text {eff }}\right] \phi_{i}(\vec{r}) & =\varepsilon_{i} \phi_{i}(\vec{r})(2.3
\end{aligned}
$$


$V_{\text {eff }}$ is defined as the sum of the $V_{\text {ext }}(\vec{r}), V_{H}(\vec{r})$, and $V_{x c}(\vec{r})$; they depend directly on the density and indirectly on the orbitals and the equation is solved self-consistently.

\subsubsection{Local Density Approximation}

In the above section, we showed how an interacting system can be solved by solving a non-interacting problem. However, the accuracy depends on how we construct the exchange-correlation potential. The old and the most popular functional is the local density approximation or LDA, which is considered to be the "mother of all density functional approximation" [11]. This approximation is based on the uniform electron gas system where the Fermi energy is defined as the highest filled planewaves and the Fermi wavevector is found to be $[17,18]$,

$$
k_{F}=\left(3 \pi^{2} n\right)^{1 / 3}
$$

In this case, the total energy is the integral over all states having wavevectors up to $\overrightarrow{k_{F}}$. So the kinetic energy per electron for each spin is [18]:

$$
\frac{T_{t o t}}{N}=\frac{\frac{1}{2} \int k^{2} d \vec{k}}{\int d \vec{k}}=\frac{3}{10} E_{F}=\frac{3}{10} \int d^{3} r k_{F}^{2} n(r)=\frac{3}{10}\left(3 \pi^{2}\right)^{2 / 3} \int n^{5 / 3} d^{3} r=2.871 \int n^{5 / 3} d^{3} r
$$


So the exchange energy for LDA can be calculated as $[11,18,17]$ :

$$
E_{x}^{L D A}[n]=A_{x} \int d^{3} r n^{4 / 3}(r)=A_{x} \int n \varepsilon_{x}^{u n i f} d^{3} r
$$

where $A_{x}$ can be found from the exchange energy per electron for a planewave, which is equal to - $0.738 ; \varepsilon_{x}^{\text {unif }}=\frac{3 k_{F}}{4 \pi}=\frac{3}{4 \pi}\left(3 \pi^{2} n\right)^{1 / 3}$. For the correlation part, we write

$$
E_{c}^{L D A}[n]=\int d^{3} r n(r) \varepsilon_{c}^{u n i f}\left(r_{s}(r)\right)
$$

where $r_{s}$ is the Wigner-Seitz radius, which is equal to $\left(\frac{3}{4 \pi n}\right)^{1 / 3}$ [we use the relation:

$\left.\frac{4 \pi}{3} r_{s}^{3}=\frac{1}{N / V}=\frac{1}{n}\right] . \varepsilon_{c}^{u n i f}$ is the correlation energy per electron for the uniform gas. Accurate exchange-correlation part is obtained by combining the limiting case of the Wigner-Seitz radius with accurate quantum Monte Carlo data. To summarize, exchange-correlation part in LDA is described by:

$$
E_{x c}^{L D A}[n]=\int d \vec{r} n(\vec{r}) \varepsilon_{x c}^{u n i f}(\vec{r})
$$

\subsubsection{Generalized Gradient Approximation (GGA)}

LDA treats the interacting system as a system of non-interacting uniform electron gas. In reality the electron distribution can be inhomogeneous. GGA which is a semi-local 
approximation, seeks to improve upon LDA by including the derivative information of the density into the exchange-correlation functional. It is expressed as $[11,18,17]$ :

$$
E_{x c}^{G G A}\left[n_{\uparrow}, n_{\downarrow}\right]=\int f\left[n_{\uparrow}, n_{\downarrow}, \nabla n_{\uparrow}, \nabla n_{\downarrow}\right] d \vec{r}
$$

GGA for the exchange is given by the following relation :

$$
E_{x}^{G G A}=\int n \varepsilon_{x}^{u n i f}(n) F_{x}(s) d \vec{r}
$$

where PBE ansatz of $F_{x}$ has the form [23]

$$
F_{x}=1+\kappa-\kappa /(1+x / \kappa)
$$

with $\kappa=0.804$ and $x=0.21951 s^{2}$

The correlation term in GGA has the following form:

$$
E_{c}^{G G A}=\int n\left[\varepsilon_{c}^{u n i f}\left(r_{s}, \zeta\right)+H\left(r_{s}, \zeta, t\right)\right] d(\vec{r})
$$

where $\zeta$ is the relative spin-polarization, $\mathrm{t}$ is a dimensionless density gradient. 


\subsubsection{B3LYP}

Hatree-Fock theory treats the exchange exactly. However it suffers from the absence of accurate correlation and can produce a wrong description of chemical bonding. In 1992, Axel. D. Becke successfully mixed the Hatree-Fock exchange and local density approximation to improve accuracy[24]. This hybrid- density functional theory incorporates Becke's three parameter exchange functional and Lee-Yang-Parr correlation functional, which is known as B3LYP. It is described as:

$$
E_{x c}^{B 3 L Y P}=E_{X C}^{L D A}+a_{0}\left(E_{x}^{H F}-E_{x}^{L D A}\right)+a_{x}\left(E_{x}^{G G A}-E_{x}^{L D A}\right)+a_{c}\left(E_{c}^{G G A}-E_{c}^{L D A}\right)
$$

where $a_{0}=0.2, a_{x}=0.72$, and $a_{c}=0.81$ are three empirical fitting parameters. 


\section{Chapter 3}

\section{Theory of Quantum Transport for}

\section{Nanoscale Systems}

\subsection{Introduction}

The theory that describes the electron transport in a macroscopic system is distinctly different than the theory that describes the phenomena for nanoscale systems. For example, the resistance in a piece of conducting wire (bulk) hardly depends on how the wire is connected to the battery. However for a nanoscale material, junction plays a very important role in conductance. More importantly, in a bulk material, the resistance is caused by the collision of electrons when they travel from one electrode to the other. In a nanoscale 
device, the channel length is shorter than the mean free path of the electron or in other words, the transport is ballistic. Now the natural question is: where does the resistance come from? The Classical physics can't explain this. We look forward to quantum mechanical theory to unravel this.

\subsection{Modeling the Device}

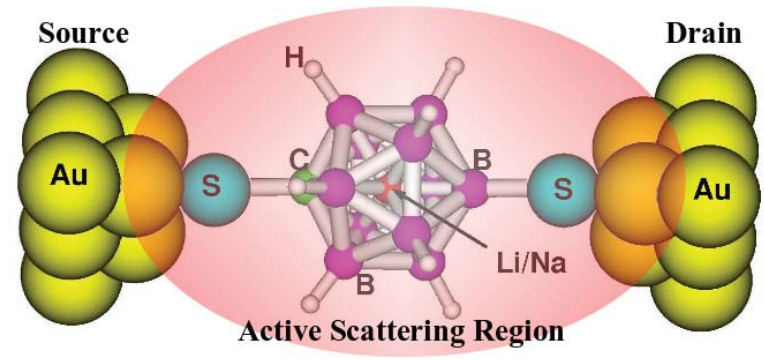

Figure 3.1: A schematic representation of nanoscale junction where a single molecule is sandwiched between two electrodes.

A typical nanojunction is shown in Fig. 3.1. A single molecule or a nanowire (spacer) is sandwiched between two semi-infinite electrodes. When a molecule or nanowire with discrete energy levels is attached to two infinite electrodes with two different occupation probabilities, the electron from one electrode (source) pumps into the molecular spacer and then pumps out and disappears into the other electrode (drain). As the device is externally connected to the battery (not shown in the figure), the process of the electron transport continues and makes the current flow possible in the circuit. Electron transport in nanoscale junction is a nonequilibrium statistical problem [25]. To solve this, we first 
assume that the current is constant over time and the energy levels in the spacer are stationary. However the molecular spacer can exchange its energy and electrons with the semi-infinite electrodes and a rigorous treatment of the device can be achieved by considering it to be an open system. To model an open nanoscale junction, we have divided it into three regions. The first one is the spacer, which consists of a molecule or nanowire with a finite length (typically around few nanometers); the second part is part of the lead that is strongly coupled to the spacer and is represented only by a finite number of atoms by which the electrodes are made of. Together with the first part and the second part is called the active scattering region. The third part is the unperturbed electrode part which is assumed to retain its bulk properties when the extended molecule is attached to the electrode. Since the electron coming into the spacer is not correlated to the electron going out from the spacer, we assume the electrochemical potential of the left electrode $\left(\mu_{L}\right)$ is different from that of the right $\left(\mu_{R}\right)[25,26]$. This difference in electrochemical potential is given by: $\mu_{1,2}=E_{f} \mp V_{\text {low,high }}$. $E_{f}$ is the equilibrium Fermi energy; $V_{\text {low }}$ and $V_{\text {high }}$ are the voltage drops at the electrodes. They are calculated self-consistently [27, 28, 29] for each applied dipole field from the difference between the average electrostatic potentials [30] at finite and zero bias at the respective electrode. The average electrostatic potential at the respective electrode (left/right) is calculated by averaging the electrostatic potential of atoms in the electrodes over the number of atoms present in each electrode (left/right) in the active region of the device. The potential difference between the source and drain, $V_{S D}$, is obtained from the difference of $V_{\text {low }}$ and $V_{\text {high }}$; at equilibrium $V_{\text {low }}=V_{\text {high }}=0$ 


\subsubsection{Looking Back from the Uncertainty Principles}

Before I describe the detailed formalism for electron transport in nano-sized junction, I would like to discuss an important feature involving the existence of the limit of conductance $[31,32]$. We know in any quantum transition the uncertainty in energy $\Delta E$ is associated with the uncertainty in time ( $\Delta t$ - during which the transition takes place) via the following uncertainty relation:

$$
\Delta E \Delta t \gtrsim \frac{\hbar}{2} \Rightarrow \frac{\Delta E}{e} \frac{\Delta t}{e} \gtrsim \frac{\hbar}{2 e^{2}}
$$

The second term in the above equation is associated with one electron current $I_{e}=e / \Delta t$ when the electron is under an external voltage of $V_{e}=\Delta E / e$. So the above equation tells

us that $V_{e} / I_{e} \gtrsim \frac{\hbar}{2 e^{2}}$ or, $G_{0} \lesssim \frac{2 e^{2}}{\hbar}$. From the above inequality, we can say that one electron moving in a single channel can have a maximum conductance of $G_{0}$ which is equal to $\frac{1}{12.9 K \Omega}$. An interesting point to note that the conductance can be viewed as the velocity of electron in Gaussian unit. Thus the upper limit of conductance is nothing but the upper limit of the speed of the electron, which is the speed of light. 


\subsection{Landauer Approach}

The Landauer approach $[33,34,26,25]$ to the quantum transport has been very successful in recent times. To discuss it in detail, let's first assume that the leads are identical at both sides and contains electrons which can freely travel in the $\mathrm{x}$-direction, but confined in other directions. The Schrodinger equation for the system is the following:

$$
\left[-\frac{\hbar^{2}}{2 m} \nabla^{2}+V(\mathbf{r})\right] \phi_{i k_{i}}(\mathbf{r})=E_{i} \phi_{i k_{i}}(\mathbf{r})
$$

The solution for the wavefunction [25]:

$$
\phi_{i k_{i}}(\mathbf{r})=\sqrt{\frac{1}{L_{x}}} u_{i}(\mathbf{r}) e^{i k x}, \quad-\infty<k<+\infty
$$

with energies

$$
E_{i}\left(k_{i}\right)=\varepsilon_{i}+\frac{\hbar^{2} k_{i}^{2}}{2 m}
$$

where $m$ is the mass of the electron and $k$ is the wavevector and $L_{x}$ is normalized length. Let's calculate the average current $I_{i}\left(E_{i}\right)$ carried by the state at energy $E_{i}$ associated with 
this wavefunction [25].

$$
\begin{aligned}
& I_{i}\left(E_{i}\right)=\left\langle\phi_{i k_{i}}|\hat{I}| \phi_{i k_{i}}\right\rangle \\
& =e\left\langle\phi_{i k_{i}}|\hat{\mathbf{j}}(\mathbf{r})| \phi_{i k_{i}}\right\rangle \\
& =\frac{e \hbar}{2 i m} \int_{-\infty}^{\infty} d y \int_{-\infty}^{\infty} d z\left[\phi_{i k_{i}}(\mathbf{r})^{*} \frac{\partial \phi_{i k_{i}}(\mathbf{r})}{\partial x}-\phi_{i k_{i}}(\mathbf{r}) \frac{\partial \phi_{i k_{i}}^{*}(\mathbf{r})}{\partial x}\right] \\
& =\frac{e \hbar k_{i}}{m L_{x}} \\
& =\frac{e v_{i}\left(k_{i}\right)}{L_{x}}
\end{aligned}
$$

Here, $\hat{\mathbf{j}}(\mathbf{r})$ is the probability current density, $v_{i}$ is the velocity with wave vector $k_{1}$. Similarly, current reflected back into the left lead is:

$$
\begin{array}{r}
I_{f}\left(E_{i}\right)=\frac{e \hbar k_{f}}{m L_{x}} \\
=\frac{e v_{f}\left(k_{f}\right)}{L_{x}}
\end{array}
$$


where $K_{f}$ points towards the negative x-direction with velocity $v_{f}$. The current deep inside the left lead is thus:

$$
I_{L}\left(E_{i}\right)=I_{i}\left(E_{i}\right)\left[1-\sum_{f=1}^{N_{L}} R_{i f}\left(E_{i}\right)\right]
$$

where $N_{L}\left(N_{R}\right)$ is the number of the channel at the energy $E_{i}$ in the left(right) electrode and $R_{i f}$ is the reflection probability, defined by the following quantity:

$$
R_{i f} \equiv\left|\mathscr{R}_{i f}\right|^{2} \frac{\left|I_{f}\left(E_{i}\right)\right|}{\left|I_{i}\left(E_{i}\right)\right|}
$$

Now the current deep inside the right lead

$$
I_{R}\left(E_{i}\right)=I_{i}\left(E_{i}\right) \sum_{f=1}^{N_{R}} T_{i f}\left(E_{i}\right),
$$

where

$$
T_{i f} \equiv\left|\mathscr{T}_{i f}\right|^{2} \frac{\left|I_{f}\left(E_{i}\right)\right|}{\left|I_{i}\left(E_{i}\right)\right|}
$$

where $\mathscr{R}_{\text {if }}$ and $\mathscr{T}_{\text {if }}$ are complex numbers.

Total current: Now the total current is the sum over all the channel and all the energies. We can get the total energy by multiplying by density of states $\left(D_{i}\left(E_{i}\right)\right)$ and integrate over 
energy. The density of states for a one-dimensional problem like this can be expressed as:

$$
D_{i}\left(E_{i}\right)=\frac{L_{x}}{2 \pi} \frac{d k_{i}}{d E_{i}}=\frac{L x}{2 \pi \hbar v_{i}\left(k_{i}\right)}
$$

So the total current is (with the factor 2 for spin degeneracy) [25, 34]:

$$
\begin{array}{r}
I=2 \int d E\left[\sum_{i=1}^{N_{L}} \sum_{f=1}^{N_{R}} f_{L} D_{i}\left(E_{i}\right) I_{i}\left(E_{i}\right) T_{i f}\left(E_{i}\right)-\sum_{i=1}^{N_{R}} \sum_{f=1}^{N_{L}} f_{R} D_{i}\left(E_{i}\right) I_{i}\left(E_{i}\right) T_{i f}\left(E_{i}\right)\right] \\
=\frac{e}{\pi \hbar} \int d E\left[f_{L}(E) \tau_{L R}(E)-f_{R}(E) \tau_{R L}(E)\right] \\
=\frac{2 e}{h} \int d E\left[f_{L}(E)-f_{R}(E)\right] T(E)
\end{array}
$$

In the above equation, we have used the conservation of flux, that is, the transmission coefficient from left to right $\left(\tau_{R L}(E)\right)$ must be equal to that for the right to left $\left[\tau_{L R}(E)=T(E)=\tau_{R L}\right] . \quad f_{L}$ and $f_{R}$ are the Fermi distribution function which can be expressed as:

$$
f_{L, R}=\frac{1}{e^{\left(E-\mu_{L, R}\right) / k_{B} T}+1}
$$




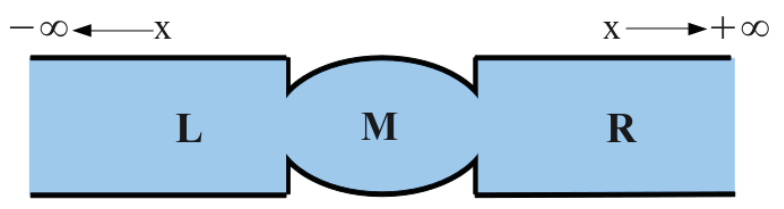

Figure 3.2: A schematic representation of a sample (M) sandwiched between two semi-infinite electrodes

\subsection{Green's Function and Self Energy}

Let's assume $H_{L}$ and $H_{R}$ to be the Hamiltonian for the isolated semi-infinite electrodes on the left and on the right region. $\mathrm{L}$ and $\mathrm{R}$ regions are coupled by the middle region $\mathrm{M}$. If $\mathrm{L}$ and $\mathrm{M}$ are coupled by a potential $\left(V_{L M}+V_{L M}^{\dagger}\right)$, and $\mathrm{M}$ and $\mathrm{R}$ are coupled by the potential $\left(V_{M R}+V_{M R}^{\dagger}\right)$, then the total Hamiltonian for the system is:

$$
H=H_{L}+H_{R}+H_{M}+V_{L M}+V_{L M}^{\dagger}+V_{M R}+V_{M R}^{\dagger},
$$

where $H_{M}$ is the Hamiltonian for the middle region. The Schrodinger equation in matrix form can be described in the following way [25, 35]:

$$
\left[\begin{array}{ccc}
H_{L} & V_{L M} & 0 \\
V_{L M}^{\dagger} & H_{M} & V_{M R}^{\dagger} \\
0 & V_{M R} & H_{R}
\end{array}\right] \times\left[\begin{array}{c}
\left|\Phi_{L}\right\rangle \\
\left|\Phi_{M}\right\rangle \\
\left|\Phi_{R}\right\rangle
\end{array}\right]=E \times\left[\begin{array}{c}
\left|\Phi_{L}\right\rangle \\
\left|\Phi_{M}\right\rangle \\
\left|\Phi_{R}\right\rangle
\end{array}\right]
$$


Where $E$ is the injection energy of the tunneling electron. Solving the above set of equations, we get:

$$
H_{L}\left|\Phi_{L}\right\rangle+V_{L M}\left|\Phi_{M}\right\rangle=E\left|\Phi_{L}\right\rangle \Longrightarrow\left|\Phi_{L}\right\rangle=G_{L} V_{L M}\left|\Phi_{M}\right\rangle,
$$

and ,

$$
\left|\Phi_{R}\right\rangle=G_{R} V_{M R}\left|\Phi_{M}\right\rangle
$$

where

$$
G_{L, R}=\frac{1}{E-H_{L, R}}
$$

From the eq. 3.22,

$$
V_{L M}^{\dagger}\left|\Phi_{L}\right\rangle+H_{M}\left|\Phi_{M}\right\rangle+V_{M R}^{\dagger}\left|\Phi_{R}\right\rangle=E\left|\Phi_{M}\right\rangle
$$

Using eq. 3.23 and eq. 3.24 we get from eq. 3.22 ,

$$
\begin{array}{r}
V_{L M}^{\dagger} G_{L} V_{L M}\left|\Phi_{M}\right\rangle+H_{M}\left|\Phi_{M}\right\rangle+V_{M R}^{\dagger} G_{R} V_{M R}\left|\Phi_{M}\right\rangle=E\left|\Phi_{M}\right\rangle \\
\left(E-H_{M}-\Sigma_{L}-\Sigma_{R}\right)\left|\Phi_{M}\right\rangle=0
\end{array}
$$


The above equation (3.28) is associated with the Green's function

$$
G(E)=\frac{1}{\left[E-H_{M}-\Sigma_{L}-\Sigma_{R}\right]}
$$

where $\Sigma_{L, R}$ are the self-energy functions for left and right electrodes [25, 26]. They are defined as:

$$
\begin{aligned}
& \Sigma_{L}=V_{L M}^{\dagger} G_{L} V_{L M} \\
& \Sigma_{R}=V_{M R}^{\dagger} G_{R} V_{M R}
\end{aligned}
$$

Eq. 3.29 can not be uniquely specified without a boundary condition. We define two different Green's functions for two different regions $[25,26]$. One is the advanced Green's $\left(G^{+}\right)$function for the region $x^{\prime}>x$, where the wavefunction at $\mathrm{x}$ is a result of an excitation at $x^{\prime}$ and another is the retarded Green's function $\left(G^{-}\right)$for the region $x^{\prime}<x$. So,

$$
G^{+}(E)=\frac{1}{E-H_{M}-\Sigma_{L}^{+}-\Sigma_{R}^{+}}, G^{-}(E)=\frac{1}{E-H_{M}-\Sigma_{L}^{-}-\Sigma_{R}^{-}}
$$


and

$$
\begin{array}{ll}
\Sigma_{L, R}^{+}(E) \equiv \Sigma_{L, R}(E+i \varepsilon) & \text { advanced } \\
& \\
\Sigma_{L, R}^{-}(E) \equiv \Sigma_{L, R}(E-i \varepsilon) & \text { retarded }
\end{array}
$$

It is important to note that advanced and the retarded self-energy operators are not Hermitian. Rather they are conjugate to each other.

$$
\left[\Sigma_{L, R}^{+}(E)\right]^{\dagger}=\Sigma_{L, R}^{-}(E)
$$

Thus through the non-Hermitian properties of the self-energies, it is easy to understand that electrons gain a finite 'lifetime' in the active scattering region before they disappear into the electrodes $[25,26]$.

Thus, we have now converted the problem for the entire system into the problem of the middle (active) region, which is open to the electrodes through the self-energy functions [25]. The energy levels in the active scattering region get broadened when it is attached to the semi-infinite electrodes and the broadening functions $\left(\Gamma_{L, R}\right)$ are related to the self-energy functions via the following relation $[25,26]$ :

$$
\Gamma_{L, R}(E)=i\left[\Sigma_{L, R}^{+}-\Sigma_{L, R}^{-}\right]
$$


Finally, the transmission function is related to the Green's function and the broadening function as $[25,26]$ :

$$
T(E)=\operatorname{Tr}\left[\Sigma_{L} G^{-} \Sigma_{R} G^{+}\right]=\operatorname{Tr}\left[\Sigma_{L} G \Sigma_{R} G^{\dagger}\right]
$$

Next, I will discuss how to obtain the coupling matrices in eq. 3.30 and 3.31 and $H_{M}(E)$ from the DFT calculations. It is important to note that in order to include the bias effect explicitly into the self-consistent calculations, we need to express the Hamiltonian, coupling matrices, and the Green's function as functions of the bias. $H_{M}(E, \varepsilon)$ is the bias dependent Kohn-Sham molecular Hamiltonian obtained by suitable partitioning of the total Hamiltonian $(H(E, \varepsilon))$ of active scattering region. The use of the real space approach for the active scattering region allows us to partition the $H(E)$ to obtain $H_{M}(E) . S_{M M}$ is the molecular overlap matrix. $\Sigma_{L, R}(E)$ are the bias dependent self-energy functions, which can be rewritten from eq. 3.30 and 3.31:

$$
\Sigma_{L, R}(E)=V_{L M, M R}^{\dagger}\left[G_{L, R}(E)\right]_{n \times n} V_{L M, M R}
$$

where $V_{L M, M R}$ are the bias-dependent molecule-lead coupling matrices and can be expressed as:

$$
V_{L M}=E \times S_{L M}-H_{L M} ; \quad V_{M R}=E \times S_{M R}-H_{M R}
$$


$H_{L M}\left(H_{M R}\right)$ and $S_{L M}\left(S_{M R}\right)$ are the electrode-molecule block of the Hamiltonian and overlap matrices on the left(right). Green's functions of the leads in the wide band approximation $[36,37,35]$ are obtained by the following relation:

$$
G_{L, R}=-i \pi \eta(E) \times I_{n}
$$

$I_{n}$ is an identity matrix of dimension $n \times n ; n$ is the total number of Gaussian basis functions used to represent the atoms of the electrode in the active scattering part of the device. A periodic DFT is used to obtain $\eta(E)$; it is calculated as DOS(E) per electron in the unit cell, where $\operatorname{DOS}(E)$ is the bulk (3D) density of states of atoms in the electrodes (typically made of gold or nickel). For DOS (E), the energy grid is taken as $0.001 \mathrm{eV}$. The use of periodic DFT to obtain the DOS(E), maintains the flavor of the electrodes being semi-infinite.

\subsection{Spin-dependent Transport}

For the spin-dependent transport, the modeling of the device is essentially the same as for the spin-independent case, except from the fact that we need to include electron's spin degrees of freedom. For the spin-dependent case, the electrodes are made of ferromagnetic materials, like nickel. Depending on the direction of the magnetization at the two electrodes, we have two configurations: one when the direction of magnetization at two electrodes are parallel (PC) and the other when they are anti-parallel (APC). In this case, the 
Hamiltonians, electron's wavefunction, self-energy functions and Green's function become spin-dependent and we need to use spin-unrestricted density functional theory [17]. Now eq. 3.29 will read as:

$$
G^{\sigma}(E, \varepsilon)=\left[E-H_{M}^{\sigma}(\varepsilon)-\Sigma_{L}^{\sigma}(\varepsilon)-\Sigma_{R}^{\sigma}(\varepsilon)\right]^{-1}
$$

$H_{M}^{\sigma}(\varepsilon)$ is now the bias dependent Kohn-Sham molecular Hamiltonian obtained by suitable partitioning of $H^{\sigma}(\varepsilon)$.

$\Sigma_{L, R}^{\sigma}(\varepsilon)$ are the bias dependent, spin-polarized self-energy functions, which are calculated as:

$$
\Sigma_{L, R}^{\sigma}(\varepsilon)=V_{L M, M R}^{\sigma \dagger}\left[G_{L, R}^{\sigma}(E)\right]_{n \times n} V^{\sigma}{ }_{L M, M R}
$$

where $V_{L M}^{\sigma}$ and $V_{M R}^{\sigma}$ are the bias-dependent molecule-lead coupling matrices. These matrices are expressed as:

$$
V_{L M}^{\sigma}=E \times S_{L M}-H_{L M}^{\sigma} ; \quad V_{M R}^{\sigma}=E \times S_{M R}-H_{M R}^{\sigma}
$$

$H_{L M}^{\sigma}\left(H_{M R}^{\sigma}\right)$ and $S_{L M}\left(S_{M R}\right)$ are the spin-dependent electrode-molecule block of the Hamiltonian and overlap matrices on the left(right). $G_{L, R}^{\sigma}$ are the spin-dependent Green's functions of the leads obtained using wideband approximation [36, 37]. When the direction 
of magnetization at the electrodes are parallel to each other,

$$
G_{L}^{\sigma}(E)=-i \pi \eta^{\sigma}(E) \times I_{n}, G_{L}^{\sigma}(E)=G_{R}^{\sigma}(E)
$$

and when the direction of the magnetization are anti-parallel, we have the following cases: for $\sigma=\uparrow$,

$$
G_{R}=-i \pi \eta^{\downarrow}(E) \times I_{n} ; G_{L}=-i \pi \eta^{\uparrow}(E) \times I_{n}
$$

and for $\sigma=\downarrow$,

$$
G_{R}=-i \pi \eta^{\uparrow}(E) \times I_{n} ; G_{L}=-i \pi \eta^{\downarrow}(E) \times I_{n}
$$

Like the spin-independent case, $I_{n}$ is an identity matrix of dimension $n \times n ; n$ is the total number of Gaussian basis functions used to represent the Ni atoms in the active scattering part of the device. Again we use periodic DFT to obtain $\eta^{\sigma}(E)$; it is calculated as DOS(E) per electron in the unit cell, where $\operatorname{DOS}(\mathrm{E})$ is the spin-polarized bulk (3D) density of states of nickel. We have aligned the Fermi energy level of the active region of the device at the equilibrium condition with the Fermi energy of the bulk Nickel. The Fermi energies of the active region for PC and APC are taken as the energies of their respective highest occupied molecular orbitals (HOMO). In case of PC and APC, we found the HOMO corresponds 
to a spin-down state. It should be noted that the finite part of the Ni electrode on each side in the active scattering region and the respective unperturbed semi-infinite part of the electrode are assumed to have the same magnetic domain. 



\section{Chapter 4}

\section{Charge Transport in a Zero-dimensional}

\section{Codoped Molecular Junction}

Portion of this chapter is copied from the Physical Review B. vol. 83, page - 195420, year -2011 by Subhasish Mandal and Ranjit Pati. Copyright - Appendix B.

\subsection{Motivation}

Doping is a scheme for tuning electronic, magnetic, and optical properties of materials by purposely introducing a small amount of impurities. It has been playing a fundamental role in the rapid growth of silicon based semiconductor technologies. Several groups 
have already started looking at the doping scheme via exploring the role of dopants in modulating the electronic structure of nanocrystals and molecular nanostructures $[38,39,40,41,42,43]$. The strong quantum confinement effect makes these nanomaterials highly sensitive to dopant atoms. A profound impact on the electronic structure causing dramatic changes in the electrical properties of these materials has been found by doping even a single atom in to the host. A well-known example is the superconductive nature found in alkali doped $C_{60}[44,45,46,47]$. It has been revealed that a dopant atom changes the electronic structure via donating or accepting charge from the host system [48].

However the "conventional controlled-doping of a single atom" [49] has been found to be exceedingly difficult particularly within a molecular length scale. To overcome such difficulties, researchers discovered a variety of synthetic approaches [50] resulting in the synthesis of a range of new molecular compounds [51]. One good example is the synthesis of polyhedral closo-boranes and closo-carborane structures [52, 53, 54, 55]. These important classes of boron-rich structures have been explored as boron carriers in boron-neutron-capture therapy and as molecular probes in medical diagnostics $[56,57,58]$. Researchers have also studied electron transfer properties of 10-, and 12-vertex carborane structures for their possible application in electronics [59]. Despite their rich chemistry, these inorganic cage structures have never been thought of to be large enough for encapsulating a dopant atom. It was first shown by Jemmis and co-workers [60] that closo-boranes can be doped endohedrally to enhance their stability. Subsequently, Oliva and colleagues have used high level quantum chemistry calculations to demonstrate that the 
Lithium encapsulated carborane structure $\left(\mathrm{Li} @ \mathrm{C}_{1} B_{11} H_{12}\right)[61]$ can be used as an effective mass selective conveyor via photochemical switching [62]. These pioneering works suggest that a rich fundamental science remains to be explored for such small inorganic cage structures. For example: How would doping a single atom affect the electronic structure of such a small inorganic host? Can a single atom play a vital role in controlling the flow of electrons in a molecular junction?

\subsection{Codoping Approach in a Single Molecular Junction}

To answer some of the above mentioned subtle questions, we have considered a precursor 1, 12-dicarba-closo-dodecaborane complex and used a codoping model by replacing one of the vertex carbon atoms by a boron atom and decorating it with an endohedrally doped, electron rich, alkali atom ( $X @ C_{1} B_{11} H_{12}, \mathrm{X}=\mathrm{Li}$ or $\mathrm{Na}$ ) to investigate the role of dopant on its electronic structure. This codoping approach allows us to maintain the charge neutrality of the molecular complex. It should be noted that this type of codoping model, where a cation and an anion are simultaneously introduced into the host, has been adopted to tune the optical properties of $\mathrm{TiO}_{2}$ semiconductors[63]. Subsequently, prototype two-terminal device structures are built from each of these individual molecules by sandwiching them between two gold electrodes, and a parameter free, first-principles, nonequilibrium Green's function approach is used to study their current-voltage characteristics. The commonly used thiolate (-S) anchoring groups are used to attach the molecule between 
the gold electrodes. Our calculations reveal that the conductivity in the $X @ C_{1} B_{11} H_{12}$ junction, where $\mathrm{X}=\mathrm{Li}$ or $\mathrm{Na}$, is significantly higher than that in the $C_{2} B_{10} H_{12}$ junction. Further analysis shows that the dopant alkali atom donates electronic charges to the cage $\left(C_{1} B_{11} H_{12}\right)$, resulting in a profound effect on its electronic structure, and therefore on its conductivity - opening up a path toward a single-atom-controlled device.

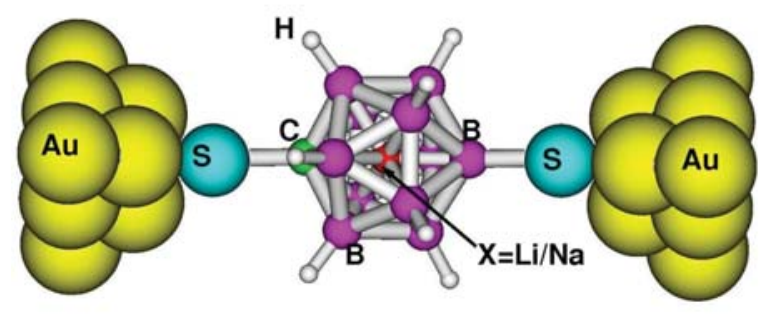

Figure 4.1: Schematic representation of alkali $(\mathrm{X}=\mathrm{Li}, \mathrm{Na}) / \mathrm{B}$-codoped carborane junction. Reprinted figure with permission from Subhasish Mandal and Ranjit Pati, Phys. Rev. B. 83195420 (2011). (c) The American Physical Society.

\subsection{Computational Methods}

We have used 1, 12-dicarba-closo-dodecaborane $\left(C_{2} B_{10} H_{12}\right)$ molecule as a precursor (Fig.4.1), and have adopted a simple codoping model to introduce atomic impurities into the host. We replaced one of the vertex carbon atoms in $C_{2} B_{10} H_{12}$ by a boron atom and decorated it simultaneously with an endohedrally doped alkali atom $\left(X @ C_{1} B_{11} H_{12}, \mathrm{X}=\mathrm{Li}\right.$ or $\mathrm{Na}$ ) to maintain the charge neutrality of the molecular end-product (Fig. 4.1 and Fig. 
4.2). The geometry optimizations are performed using the density functional theory, which involves Becke's three parameter hybrid functional (B3LYP) [64, 65] for exchange and correlation. A real space approach, in which the many-body wavefunction of the electron is expanded in terms of a finite set of Gaussian atomic orbitals [65], is used for this calculation. We have utilized a triple valence zeta basis set augmented by polarization and diffuse functions $\left(6-311++\mathrm{G}^{* *}\right)$ to carry out these calculations.

Next, a prototypical two-terminal molecular device is built from each of these molecular complexes by sandwiching them between two semi-infinite gold electrodes; thiolate (-S) anchoring groups are used to attach the molecule between the electrodes (Fig. 4.1). The first part is the active scattering region, which consists of the molecular part of the device and a finite number of gold atoms from the Au (111) surface. Particularly, we have embedded the molecule with thiolate (-S) anchoring groups in between two clusters of three $\mathrm{Au}$ atoms each; $\mathrm{S}$ is incorporated into the three-fold hollow site of the $\mathrm{Au}$ atoms $[10,66]$. For the gold atoms, the Los Alamos double zeta effective core potential basis set [65] that includes the scalar relativistic effects is used. During self-consistent calculation, to ensure tight convergence, the convergence criterion for energy, maximum, and root mean square electron density are set at $10^{-6}, 10^{-6}$, and $10^{-8}$ a.u. respectively.

It should be noted that the ground state based DFT approach, which is used here to evaluate $H(\varepsilon)$, has limitations in predicting the excitation energy. Accurate description of the excitation requires approaches beyond mean field theory [67] such as configuration 
interaction, [68, 69] coupled cluster [70] or GW approach [71]. Some of these higher level methods though have been explored in the context of transport in molecular junctions, their complete implementation is prohibitively difficult both in the time independent or the time dependent framework; the time dependent formalism would be more appropriate $[72,25,73,74,75,76,77]$. It is important to note that the transport in molecular junction not only requires the accurate energy level description of the molecular spacer but also it depends upon the precise determination of the coupling between the molecule and the semi-infinite electrode. Particularly, for the later case, the higher level approaches are difficult to implement. In addition, the requirement to include bias effect self consistently compounds the difficulty. The ground state based DFT approach $[140,79,80,74,81,82$, $83,84,85,28]$ has been quite successful in explaining the experimental results qualitatively, and quantitatively in some instances. Here, we have considered a strongly coupled, chemically bonded junction, where the coupling between molecule and lead plays a more dominant role. In such a scenario, ground state based DFT approach would be a reasonably good approximation to treat electronic current.

As discussed in chapter3, we have implemented the single-particle Green's function approach to investigate charge transport properties. In this formalism, the nonequilibrium Green's function $[10,79,140,86,83,84]$, which has an implicit bias dependence.

We have added and subtracted a small thermal smearing term, $k_{B} T(=0.026)$ into the upper and lower limit of the integration in eq. 3.19 to account for the electronic temperature at 
the interface in the nonequilibrium condition.

\subsection{Electronic Structure of Undoped and Codoped}

\section{Molecules}

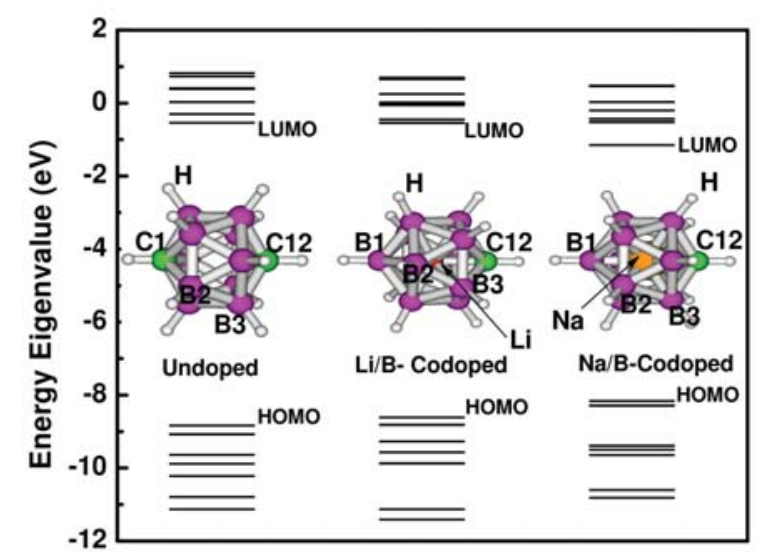

Figure 4.2: $\quad$ Energy eigenvalues of $C_{2} B_{10} H_{12}, L i @ C_{1} B_{11} H_{12}$, and $N a @ C_{1} B_{11} H_{12}$. Respective optimized structures are shown in the inset. Reprinted figure with permission from Subhasish Mandal and Ranjit Pati, Phys. Rev. B. 83195420 (2011). (C) The American Physical Society.

The optimized structures and the energy eigenvalues of undoped and doped molecules are presented in Fig. 4.2. The structural details including the formation energy $\left(\Delta_{F E}\right)$ are summarized in Table 4.1. The formation energy is calculated as $\Delta_{F E}=\left(E_{M O L}-\Sigma E_{I}\right) / \Sigma_{I}$, where $E_{M O L}$ is the total energy of the molecule; $E_{I}$ is the energy of the atom present in the molecule and $\Sigma_{I}$ is the total number of atoms. It is clear from the values of $\Delta_{F E}$ (Table 4.1) that these three systems are stable. One can also notice from Table 4.1 that 
the bond lengths of $p$-carborane $\left(p-C_{2} B_{10} H_{12}\right)$ compare very well with the previously reported theoretical and experimental values. The electron diffraction study reported the structure of $p$-carborane to be slightly distorted from the icosahedron symmetry with $\mathrm{C}(1)-\mathrm{B}(2)$ and $\mathrm{B}(2)-\mathrm{B}(3)$ bond lengths of $1.710 \AA$ and $1.792 \AA$ respectively [89]. The theoretical calculation at the MP2 level reported the $\mathrm{C}(1)-\mathrm{B}(2)$ and $\mathrm{B}(2)-\mathrm{B}(3)$ bond length to be $1.703 \AA$ and $1.781 \AA$ respectively [90]. These data are in excellent agreement with our calculated values of $1.71 \AA$ and $1.77 \AA$ for the respective bond lengths. We then calculated the energy for the positively charged state of the $p$-carborane and compared that with the energy of the neutral state to obtain the ionization potential (IP) value of $10.87 \mathrm{eV}$, which is in good agreement with the reported experimental IP of $10.6 \mathrm{eV}$ [91]. For the doped molecules, the structural details are not available for comparison. From Table 4.1, the distance between the two vertex atoms in $C_{2} B_{10} H_{12}$ is found to be $3.05 \AA$, which increases to $3.43 \AA$ in $L i @ C_{1} B_{11} H_{12}$. In $N a @ C_{1} B_{11} H_{12}$, the distance between the vertex atoms ( $\mathrm{C}$ and $\mathrm{B}$ atoms) is found to be $3.73 \AA$ A. Similar expansion in C-B and B-B bond lengths upon codoping are noted in Table 4.1. The expansion of cage structure upon codoping has important implications on their electronic structures as revealed from the eigenvalue spectrum (Fig. 4.2). Upon codoping of $\mathrm{Li}$ at the endohedral site and $\mathrm{B}$ at the substitutional site, the highest occupied molecular orbital (HOMO) level in $L i @ C_{1} B_{11} H_{12}$ shifted upwards but the lowest unoccupied molecular orbital (LUMO) level remains almost at the same position resulting in a reduction of the HOMO-LUMO gap $\left(\Delta E_{g}\right)$ from 8.29 $\mathrm{eV}$ to $8.07 \mathrm{eV}$. In $N a @ C_{1} B_{11} H_{12}$, the HOMO level shifted upwards and the LUMO level 
shifted downwards resulting in a further reduction of $\Delta E_{g}$; the $\Delta E_{g}$ was found to be 7.01 eV. To gain further insight, we carried out the Mulliken charge analysis for the undoped and doped molecules. In $p$-carborane, all the boron atoms are negatively charged and all the carbon atoms are positively charged. However in $L i @ C_{1} B_{11} H_{12}$ and $N a @ C_{1} B_{11} H_{12}$, the $\mathrm{Li}$ and $\mathrm{Na}$ have positive charges; negative charges are distributed on the rest of the $\mathrm{B}$ and $\mathrm{C}$ atoms. The strong polarization effect in $X @ C_{1} B_{11} H_{12}$, where $\mathrm{X}=\mathrm{Li}$ or $\mathrm{Na}$, suggests that these alkali atoms have a strong influence on their electronic structure.

Table 4.1

Calculated $\Delta_{F E}$ (eV/atom), bond lengths in undoped, Li/B-codoped, and $\mathrm{Na} / \mathrm{B}$-codoped carborane molecules.

\begin{tabular}{llll}
\hline Molecule & $\Delta_{F E}(\mathrm{eV} /$ atom $)$ & Atoms $^{1}$ & Bond length $(\AA)$ \\
\hline$C_{2} B_{10} H_{12}$ & -4.48 & $\mathrm{C}(1)-\mathrm{C}(12)$ & 3.05 \\
& & $\mathrm{C}(1)-\mathrm{B}(2)$ & 1.71 \\
& & $\mathrm{~B}(2)-\mathrm{B}(3)$ & 1.77 \\
\hline$L i @ C_{1} B_{11} H_{12}$ & -4.26 & $\mathrm{~B}(1)-\mathrm{C}(12)$ & 3.43 \\
& & $\mathrm{C}(12)-\mathrm{B}(3)$ & 1.79 \\
& & $\mathrm{~B}(1)-\mathrm{B}(2)$ & 1.90 \\
& $\mathrm{~B}(2)-\mathrm{B}(3)$ & 1.86 \\
& & $\mathrm{~B}(1)-\mathrm{Li}$ & 1.74 \\
& & $\mathrm{C}(12)-\mathrm{Li}$ & 1.69 \\
\hline$N a @ C_{1} B_{11} H_{12}$ & -3.55 & $\mathrm{~B}(1)-\mathrm{C}(12)$ & 3.73 \\
& & $\mathrm{C}(12)-\mathrm{B}(3)$ & 1.91 \\
& & $\mathrm{~B}(1)-\mathrm{B}(2)$ & 2.12 \\
& $\mathrm{~B}(2)-\mathrm{B}(3)$ & 1.98 \\
& $\mathrm{~B}(1)-\mathrm{Na}$ & 1.88 \\
& & $\mathrm{C}(12)-\mathrm{Na}$ & 1.86 \\
\hline
\end{tabular}




\subsection{Transport Properties of Undoped and Codoped Molecular Junctions}

Now the question arises: Will the observed strong influence of alkali atoms have a measurable effect on the conductivity of the material? To address this question, we built a prototype device (Fig. 4.1) as discussed in the previous sections. Since the atomic level structural details at the molecule-lead interface is not available a priori, we varied the $\mathrm{S}-\mathrm{Au}$ distance in the active part of the device to find the equilibrium configuration. In $p$-carborane

system, the optimized S-Au distance is found to be $2.77 \AA$. As our aim is to explicitly investigate the effect of dopant atoms on the conductivity, we have kept the interfacial contact structure the same for all systems.

\subsubsection{Potential Profile}

To model the device in the nonequilibrium condition and to understand the electronic response of the molecule, we have applied a dipole electric field along the molecular wire axis as discussed above. we have calculated the electrostatic potential (EP) self-consistently at each atomic center in the active part of the device at equilibrium and nonequilibrium conditions. In the nonequilibrium condition, EP is obtained as a function of external applied field. Subsequently, the difference of EP between the equilibrium and nonequilibrium 
situation is averaged over the degrees of freedom perpendicular to the wire axis to obtain the relative electrostatic potential (REP). The REP values at each atomic center are then plotted as a function of applied bias $\left(V_{S D}\right)$ along the molecular wire axis (Fig. 4.1). In Fig. 4.3(a), the potential profile for three systems at $V_{S D} \sim 1$ volt is presented. First, from Fig. 4.3(a), a sudden drop in potential is noticed at the Au-S junctions for the undoped carborane system. Second, the two terminal S-atoms for $C_{2} B_{10} H_{10}$ system are located at the valley and hill of the potential profile respectively, exhibiting dipole field induced polarization effect. Third, there is an effective potential barrier between the two terminal S-atoms. However, for the codoped system, at the left S-Au junction, a steady drop in potential is observed in contrast to an abrupt change in potential at the right $\mathrm{S}$-Au junction. For the codoped system, the effective barrier height between the terminal S-atoms is significantly smaller than that in the undoped system; this could have a significant effect on the conductivity. The observed asymmetric feature in potential drops at the Au-S junction in the codoped systems is due to the structural asymmetry at the vertex position; the opposite vertex atoms in the codoped systems are C and B atoms. In Figs. 4.3(b), 4.3(c), and 4.3(d), we have summarized the potential profile for undoped, $\mathrm{Li} / \mathrm{B}$-codoped, and $\mathrm{Na} / \mathrm{B}$-codoped systems respectively. For the undoped system, at $V_{S D}=1.99 \mathrm{~V}$, the magnitude of potential drop from $\mathrm{Au}$ to $\mathrm{S}$ on the left is found to be $0.69 \mathrm{~V}$; on the right $\mathrm{Au}-\mathrm{S}$ junction the potential drop is found to be $0.88 \mathrm{~V}$. In the case of $L i @ C_{1} B_{11} H_{10}$ system, at $2.06 \mathrm{~V}$, a smaller potential drop of $0.41 \mathrm{~V}$ is observed on the left Au-S junction in contrast to a larger drop of $0.60 \mathrm{~V}$ on the right. A Similar steady drop of $0.46 \mathrm{~V}$ on the left junction and a larger drop of $0.71 \mathrm{~V}$ is found in the 
$N a @ C_{1} B_{11} H_{10}$ system at $2.06 \mathrm{~V}$. A closer examination reveals a significant change in the potential profile as a function of applied $V_{S D}$, suggesting that the inclusion of bias effect is essential for the quantitative evaluation of the electronic response of the molecular device.

\subsubsection{Current-voltage}

The current for undoped, Li/B-codoped, and Na/B-codoped systems is calculated using eq. 3.19 for each applied bias. The calculated current-voltage characteristic for undoped, Li/B-codoped, and $\mathrm{Na} / \mathrm{B}$-codoped systems are summarized in Fig. 4.4. First, for the undoped system, a steady increase in current with increasing applied bias is noted. For the codoped systems, the calculated current is found to be significantly higher than the undoped system. For example, at $1.99 \mathrm{~V}$, for the undoped system, the calculated $I_{S D}$ is found to be $0.96 \mathrm{~mA}$. In the case of the $\mathrm{Li} / \mathrm{B}$-codoped system, the calculated $I_{S D}$ is found to be $4.11 \mathrm{~mA}$ at $1.93 \mathrm{~V}$ and for the $\mathrm{Na} / \mathrm{B}$-codoped system, the $I_{S D}$ is found to be 8.07 $\mathrm{mA}$ at $1.86 \mathrm{~V}$, which is $\sim 8$ times higher than that in the undoped system. The two fold increase in current from the $L i @ C_{1} B_{11} H_{10}$ system to the $N a @ C_{1} B_{11} H_{10}$ system suggests that the single alkali atom $(\mathrm{Na} / \mathrm{Li})$ plays a dominant role in controlling the conductivity of these molecular junctions; this could potentially lead to an ultimate single-atom controlled device. Further examination of Fig. 4.4 reveals a non linear feature in current above $V_{S D} \sim$ $1 \mathrm{~V}$ in the $\mathrm{Na} / \mathrm{B}$-codoped system in contrast to a linear behavior in current for the undoped and the $\mathrm{Li} / \mathrm{B}$-codoped systems. 


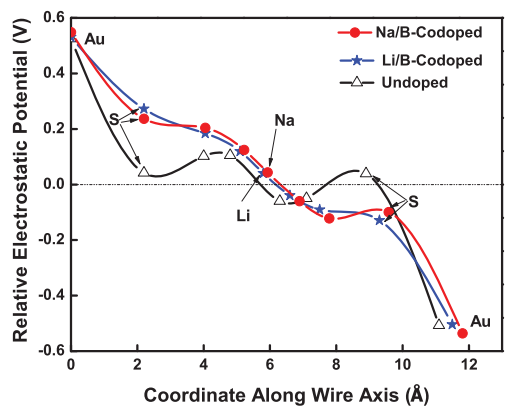

(a)

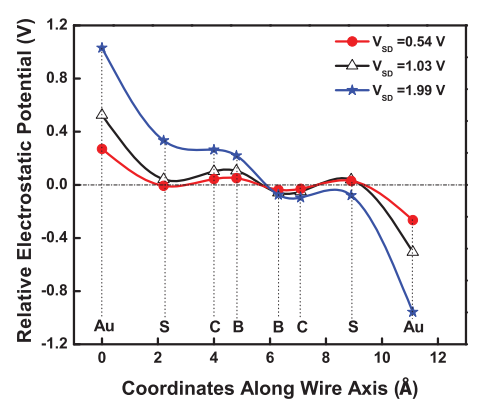

(b)

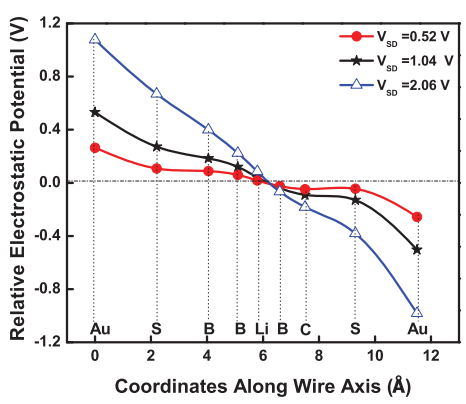

(c)

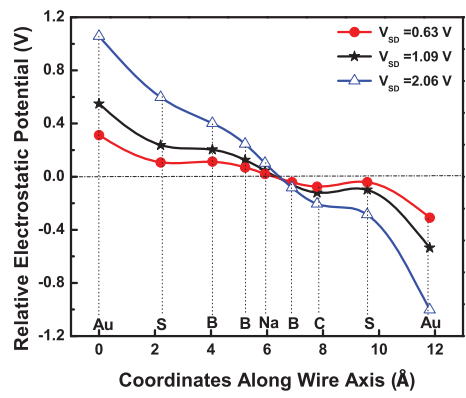

(d)

Figure 4.3: (a) Potential profile of undoped, Li/B-codoped, and $\mathrm{Na}$ /B-codoped junctions at $V_{S D} \sim 1 \mathrm{~V}$. (b) Bias dependent potential profile for undoped, (c) Li/B-codoped, and (d) Na/B-codoped junctions. The vertical doted lines depict the location of the atoms along the wire axis in the device. Reprinted figure with permission from Subhasish Mandal and Ranjit Pati, Phys. Rev. B. 83195420 (2011). (C) The American Physical Society. 


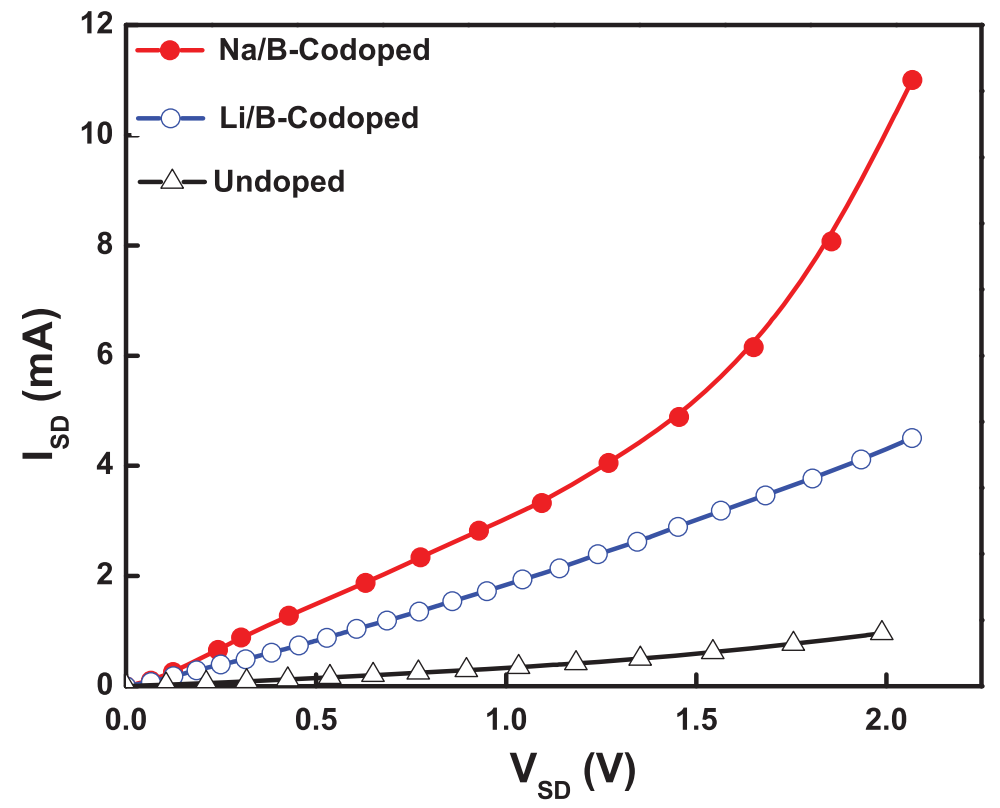

Figure 4.4: Realization of single-atom-controlled device through the current-voltage characteristic of undoped, Li/B-codoped, and $\mathrm{Na} / \mathrm{B}$-codoped junctions. Reprinted figure with permission from Subhasish Mandal and Ranjit Pati, Phys. Rev. B. 83195420 (2011). (c) The American Physical Society.

\subsubsection{Bias Dependent Transmission}

If we look at the formula (eq. 3.19), electronic current is obtained by integrating the transmission function $T(E)$. To understand the significant increase in current upon codoping and to account for the observed non-linear feature in current for the $\mathrm{Na} / \mathrm{B}$-codoped system, we need to look at the bias dependent transmission as a function of injection energy. $T(E)$ is calculated by using eq. 3.37. The results are presented 
in Figs. 4.5(a) and 4.5(b). The chemical potential window is shown by the dotted line and the Fermi energy is set to zero in the energy scale. For brevity, we have considered only two bias points. From the figures it is clear that in the $\mathrm{Na} / \mathrm{B}$-codoped system the transmission is considerably higher than that in the undoped system. For example, at $V_{S D} \sim 2 \mathrm{~V}$, the transmission in the case of $N a @ C_{1} B_{11} H_{10}$ at injection energy of $1.0 \mathrm{eV}$ is 116.85 as compared to 11.6 for the undoped system. This ten fold increase in transmission for $N a @ C_{1} B_{11} H_{10}$ as compared to the undoped system accounts for the $\sim 8$ times increase in current at $\sim 2 \mathrm{~V}$ for the former. In the Li/B-codoped system, $T\left(E=1.0, V_{S D} \sim 2 V\right)$ is found to be 42.04. At $V_{S D} \sim 1 \mathrm{~V}$, for an injection energy of $0.5 \mathrm{eV}$, the $T(E, V)$ is found to be 5.9 for the undoped system compared to 45.13 for $N a @ C_{1} B_{11} H_{10}$.

By comparing the $T(E, V)$ in Figs.4.5(a) and 4.5(b), one can notice that there is a steep increase of $T(E, V)$ with the increase of injection energy from 0 to $1 \mathrm{eV}$ in the case of $\mathrm{Na}$ /B-codoped system at $\sim 2 \mathrm{~V}$; while, in the case of the Li/B-codoped and the undoped systems, a steady increase is observed in $T(E, V)$ with the increase of injection energy. Thus, the rapidly increasing area under $T(E, V)$ with increasing bias for $\mathrm{Na} / \mathrm{B}$-codoped system is responsible for the observed nonlinear behavior of current above $\sim 1$ V. Further examination of $T(E, V)$ in Figs. 4.5(a) and 4.5(b) reveals an interesting phase shift behavior between the $\mathrm{Na} / \mathrm{B}$-codoped and $\mathrm{Li} / \mathrm{B}$-codoped systems; the injection energy for the maximum transmission in the $\mathrm{Na} / \mathrm{B}$-codoped system matches that of the minimum transmission in the $\mathrm{Li} / \mathrm{B}$-codoped system. The expansion of the cage structure in the $\mathrm{Na}$ /B-codoped system as compared to that in the Li/B-codoped system resulting in a path 
difference between the leads could possibly be the cause for the observed phase change in the transmission.

\subsubsection{Molecule-lead Coupling}

Since the $T(E, V)$ is a unified feature that depends upon both the intrinsic properties of the molecule and the electronic structure at the molecule-lead interface, we have investigated the role of molecule-lead coupling to identify the origin of increase in $T(E, V)$ in codoped systems. First, we recalculated the current at $1.99 \mathrm{~V}$ for the undoped carborane junction using $V_{L M}$ and $V_{M R}$ extracted at $0.53 \mathrm{~V}$, say $I^{\prime}$. The ratio is then calculated between the original current at $1.99 \mathrm{~V}$ and $I^{\prime}$, which is found to be 0.27 . A similar approach has been adopted to evaluate the ratio for $\mathrm{Li} / \mathrm{B}$-codoped and $\mathrm{Na} / \mathrm{B}$-codoped systems. In the case of the Li/B-codoped system, we recalculated the current at $1.93 \mathrm{~V}$ using the $V_{L M}$ and $V_{M R}$ extracted at $0.53 \mathrm{~V}$; in the $\mathrm{Na} / \mathrm{B}$-codoped system the current was recalculated at $1.85 \mathrm{~V}$ using $C_{L}$ and $C_{R}$ extracted at $0.43 \mathrm{~V}$. The ratio in current for the Li/B-codoped system is found to be 0.73 , while for the $\mathrm{Na} / \mathrm{B}$-codoped system it is 1.85 . This unambiguously suggests that the interfacial coupling changes significantly with increasing bias in the codoped systems resulting in a giant change in conductivity $(\sim$ an order in the case of the $\mathrm{Na} / \mathrm{B}$-codoped system at $\sim 2 \mathrm{~V}$ ). 


\subsubsection{Charge Profile}

To gain further insight, we have analyzed the Mulliken charge on each atom of the device in equilibrium $\left(V_{H I G H}=V_{L O W}\right)$ and nonequilibrium conditions $\left(V_{H I G H} \neq V_{L O W}\right)$. The bias dependent charge profiles at the interfacial $\mathrm{S}$ atoms for undoped, Li/B-codoped, and $\mathrm{Na} / \mathrm{B}$-codoped systems are summarized in Fig. 4.6. For the undoped system, at equilibrium, both the terminal $\mathrm{S}$ atoms have the same charge. As the bias increases, the left $\mathrm{S}$ atom steadily gains positive charge whereas the right $\mathrm{S}$ atom loses positive charge. This bias induced polarization effect accounts for the valley and the hill at the terminal S atoms in the observed potential profile of the undoped system (Fig. 4.3(a)). As expected, due to structural asymmetry at the vertex position in the codoped systems, both the terminal $\mathrm{S}$ atoms have different charges at equilibrium. The charge asymmetry at $\mathrm{S}$ atoms is much more significant in the case of the Na/B-codoped system. Interestingly, in contrast to the undoped system, the left $\mathrm{S}$ atom is found to lose positive charge while the right $\mathrm{S}$ atom is found to gain positive charge with the increase in $V_{S D}$ in codoped systems. The charge profile of $\mathrm{S}$ in the codoped systems is also reflected from the observed potential profile described in Figs. 4.3(c) and 4.3(d). To unravel the role of alkali atoms in codoped systems, we have also plotted the bias dependent Mulliken charge associated with the alkali atoms as well as the total Mulliken charge associated with $C_{1} B_{11} H_{10} S_{2}$ in Figs. 4.7(a) and 4.7(b). The charge in the charge axes is scaled by subtracting the respective charge obtained at equilibrium condition. An intriguing feature is observed by inspecting the charge profile 
(Figs. 4.7(a) and 4.7(b)). First, we found the alkali atoms are positively charged, and the negative charge is distributed over $C_{1} B_{11} H_{10} S_{2}$ in equilibrium. As bias increases, the alkali atoms gain negative charge while $C_{1} B_{11} H_{10} S_{2}$ gains positive charge, suggesting prominent charge transfer from the alkali atoms to $C_{1} B_{11} H_{10} S_{2}$ with the increase of bias.

It is important to mention that a similar charge transfer from the dopant atom to the host has been observed in a recent experiment, where $\mathrm{K}$ atoms are doped into the $C_{60}$ host [48]. In the case of the Li/B-codoped system, the loss of charge from Li atom is approximately same as the charge gain by $C_{1} B_{11} H_{10} S_{2}$. In contrast, in the case of the $\mathrm{Na} / \mathrm{B}$-copdoped system, the charge loss from $\mathrm{Na}$ is not equal to the charge gain by $C_{1} B_{11} H_{10} S_{2}$; charge loss in $\mathrm{Na}$ is minimal. Further analysis suggests that there is a strong coupling between the $\mathrm{Na}$ atom and the leads via $\mathrm{S}$ atoms. This explains the huge increase in current in the case of the $\mathrm{Na} / \mathrm{B}$-codoped system. Since a single alkali atom primarily controls the current-voltage characteristic in codoped system, we term this device as a single-atom-controlled device.

\subsection{Conclusions}

In summary, we have used a codoping model and a parameter free nonequilibrium Green's function approach in conjunction with the density functional theory to study the role of a dopant atom in strongly coupled $p$-carborane junction. When compared to the undoped system, at $\sim 2 \mathrm{~V}$, we found an order of magnitude increase in the current value in the 
$\mathrm{Na} / \mathrm{B}$-codoped system. Comparing to the current value in the Li/B-codoped system, a two fold increase in the current is observed at $\sim 2 \mathrm{~V}$ in the $\mathrm{Na} / \mathrm{B}$-codoped system; this suggests that the single alkali atom dictates the electron flow in codoped junction. Further analysis reveals that alkali atoms donate charge to the $C_{1} B_{11} H_{10} S_{2}$ host; the amount of charge transfer varies with the applied bias. This research thus opens up the door toward an ultimate limit of the miniaturization, where a single atom controls the device characteristics. 


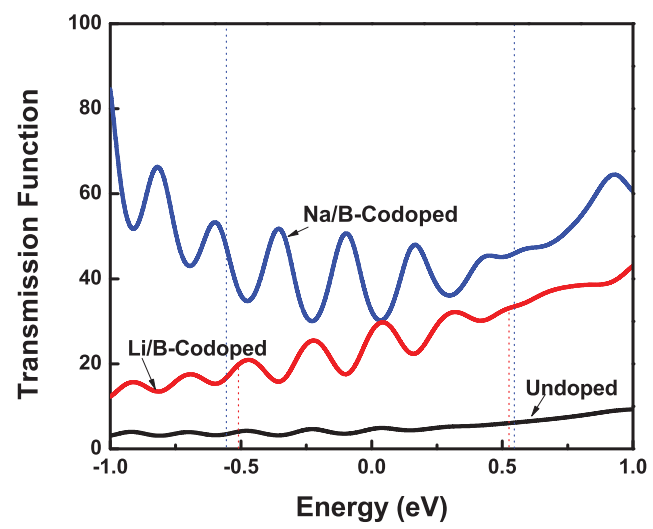

(a)

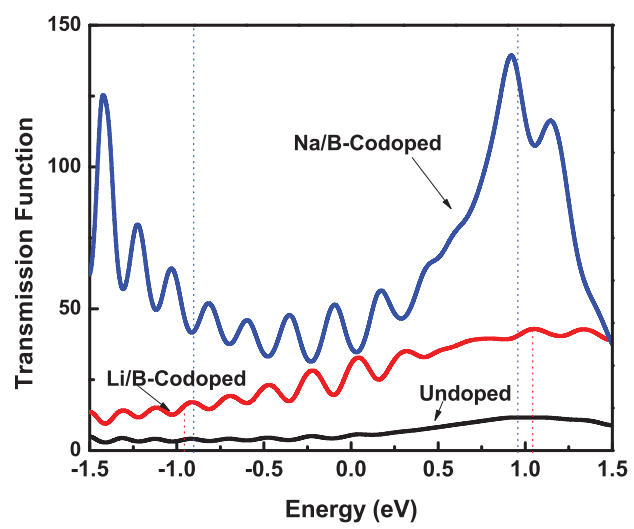

(b)

Figure 4.5: Bias dependent transmission as a function of injection energy at (a) $\sim 1$ Volt, and (b) $\sim 2$ Volt; Fermi energy is set to zero in the energy scale; dotted lines represent the chemical potential window. Reprinted figure with permission from Subhasish Mandal and Ranjit Pati, Phys. Rev. B. 83 195420 (2011). (c) The American Physical Society. 


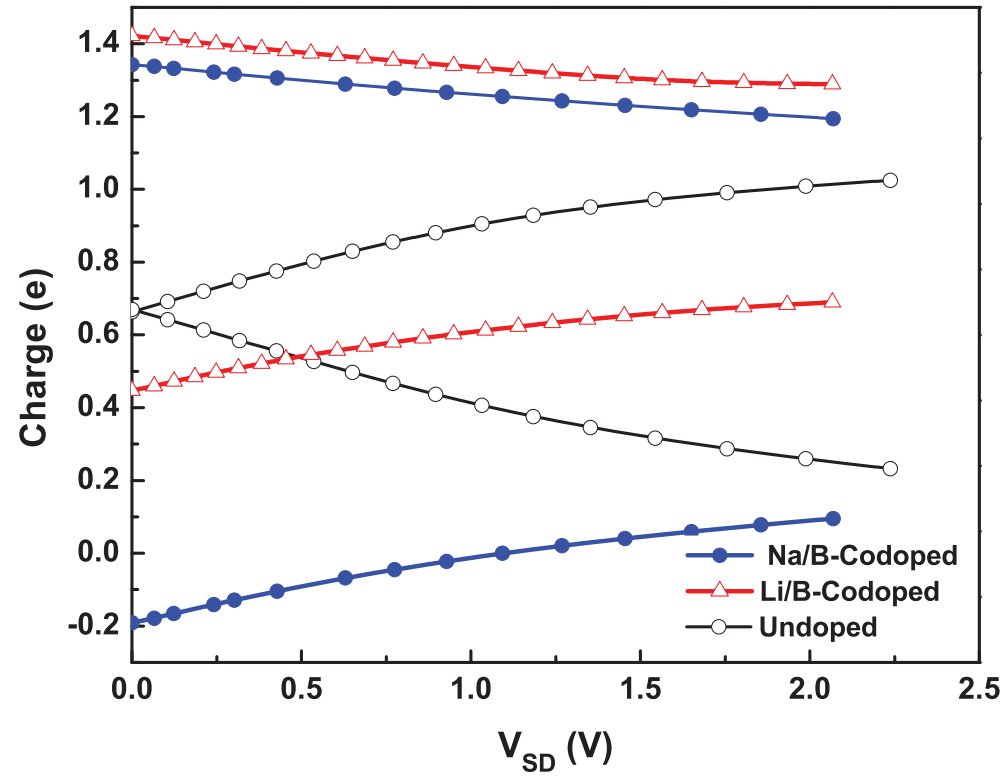

Figure 4.6: Charge profile characterizing bias dependent polarization effect on the terminal ' $S$ ' atoms. Reprinted figure with permission from Subhasish Mandal and Ranjit Pati, Phys. Rev. B. 83195420 (2011). (C) The American Physical Society. 


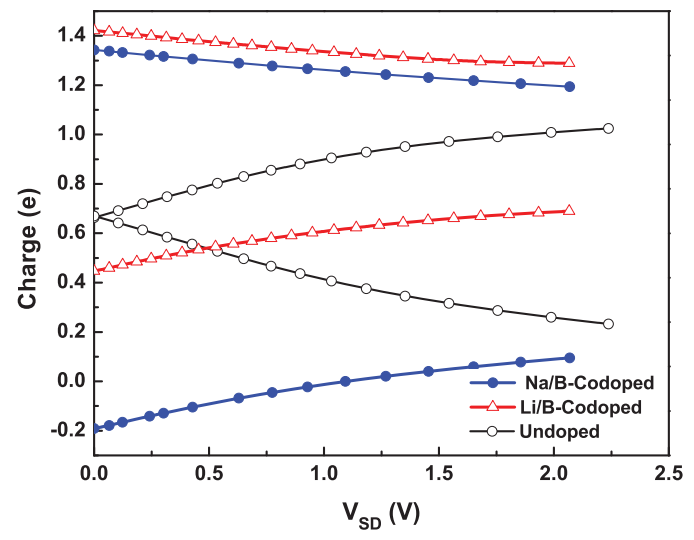

(a)

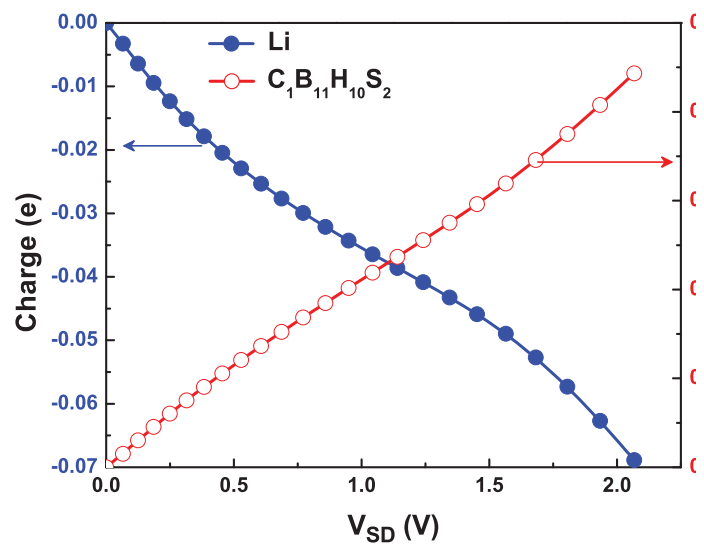

(b)

Figure 4.7: Charge profile characterizing bias dependent polarization effect between the alkali atom and the cage $\left(\mathrm{C}_{1} B_{11} H_{10} S_{2}\right)$ in (a) Li/B-codoped, and (b) Na/B-codoped junctions. Reprinted figure with permission from Subhasish Mandal and Ranjit Pati, Phys. Rev. B. 83195420 (2011). (C) The American Physical Society. 


\section{Chapter 5}

\section{Electronic Structure and Transport}

\section{Properties in One-dimensional Nanowire}

Portion of this chapter is copied from the Physical Review B, vol. 84, page - 115306, year -2011 by Subhasish Mandal and Ranjit Pati and the Chemical Physics Letter, vol. 479, page -244, year - 2009 by Subhasish Mandal and Ranjit Pati. Copyright - Appendix A and Appendix $B$. 


\subsection{Introduction}

While the size of the conventional silicon based field effect transistor is inching toward its fundamental limit of miniaturization, the quantum controlled, semi-conducting nano-wire has emerged as one of the promising options to meet the physical challenges imposed by quantum mechanics $[92,93]$. One dimensional nanowires have become the foremost candidates in building nano transistor, optoelectronic devices, nano sensors, nano electrodes, and logic circuits $[94,95,96,97,98,99]$. One of the key features that dictate their suitability in these applications is their tunable electronic structure property and hence their energy band gap. In recent years, IV-VI PbS hetero nanowire (NW) structures have drawn considerable interest for their potential applications in optical switches $[100,101]$ and solar cells $[102,103]$. Controlled synthesis of $\mathrm{PbS}$ nanowires with diameters ranging from $1.2 \mathrm{~nm}$ to $10 \mathrm{~nm}$ have been reported $[104,105,106]$. This presents exciting opportunities to explore the tunable electronic structure property of this material in the strong quantum confinement regime $[107,108,109,110,111,112] . \mathrm{PbS}$ in the bulk phase has a cubic close packed (CCP) structure with a near infrared direct band gap of $0.41 \mathrm{eV}$ at the L point [113]. Current photo luminescence study reveals a wide band gap for $\mathrm{PbS}$ nanowires due to a higher degree of quantum confinement [104]. Before going to discuss the electron transport properties in $\mathrm{PbS}$ nanowire junction, I would like to describe the electronic structure properties of one dimensional pristine $\mathrm{PbS}$ nanowire and try to find out the reason behind the observed phase transition from the first principles. This chapter 
is divided in two parts. First, we will discuss the electronic structure properties in $\mathrm{PbS}$ nanowire followed and then will discuss the charge transport properties in $\mathrm{PbS}$ nanowire junction.

\subsection{Quantum Confinement Effect in PbS Nanowire}

Despite the progress made in the last few years in the controlled synthesis of $\mathrm{PbS}$ nanowires, no theoretical calculations have been reported on these systems to understand the quantum confinement effect and the origin of the observed phase transition phenomenon. We have made the first attempt to elucidate the tunable confinement effect in the $\mathrm{PbS}$ nanowire as well as the origin of the phase transition from electronic structure calculations. We have used the first principles density functional theory to study the variation of the energy band gap $\left(\Delta E_{g}\right)$ with the diameter of the $\mathrm{PbS}$ nanowire. By varying the diameter(d) of the nanowire from $\sim 1.17 \mathrm{~nm}$ to $\sim 3.64 \mathrm{~nm}$, the $\Delta E_{g}$ is found to change from $1.524 \mathrm{eV}$ to $0.955 \mathrm{eV}$; this is substantially higher than the $\Delta E_{g}$ of $\sim 0.4 \mathrm{eV}$ observed for the bulk $\mathrm{PbS}$ - clearly revealing the role of the quantum confinement. The reduced $\mathrm{Pb}-\mathrm{S}$ bond length in the $\sim 1.17 \mathrm{~nm}$ diameter $\mathrm{NW}$ as compared to that in the $\sim 3.64 \mathrm{~nm}$ wire leads to a more confined charge density and is found to be responsible for the observed increase in the $\Delta E_{g}$ with the decrease of 'd' of the wire. 


\subsubsection{Computational Methods}

As atomic level structural details of the PbS NWs are not available a priori, we used the $\mathrm{CCP} \mathrm{PbS}$ structure as the guiding point and constructed the $1 \mathrm{D}$ nanowire in the observed (200) growth direction. we selected two layers from the bulk structure with twelve $\mathrm{Pb}$ atoms and twelve $\mathrm{S}$ atoms and placed them in a tetragonal unit cell with guess lattice parameter $c$ along the z-axis to construct the unit cell of the NW [115]. To avoid spurious interaction between the NW and its periodic images in the $\mathrm{x}$ and $\mathrm{y}$ - directions, we have taken a relatively large lattice parameter with a $0.72 \mathrm{~nm}$ of vacuum space along those two directions. Subsequently, the NW structure is optimized. The optimum value of $c$ is found to be $0.60 \mathrm{~nm}$. A similar procedure is used to obtain the equilibrium NW structure for the other three NWs of $\mathrm{d} \sim 1.98 \mathrm{~nm}, \sim 2.80 \mathrm{~nm}$, and $\sim 3.64 \mathrm{~nm}$, containing 64, 120, and 192 atoms in the unit cell respectively. The optimum $c$ value $(0.60 \mathrm{~nm})$ is found to be insensitive to the diameter of the NW. The lattice parameter for the $\mathrm{PbS}$ nanowire of diameter $\sim 100$ $\mathrm{nm}$ reported from a recent experiment [114] is $\sim 0.597 \mathrm{~nm}$, which is in excellent agreement with our calculated value of $0.60 \mathrm{~nm}$. The average distance between two $\mathrm{Pb}$ atoms or two $\mathrm{S}$ atoms located at two corners of the optimized unit cell (shown in Fig. 5.1 by the dotted line) is calculated, and the covalent radii of $\mathrm{Pb}$ and $\mathrm{S}$ are added appropriately to the average distance to estimate the diameter of the NW [115]. We have used the plane wave basis functions and periodic density functional approach using the generalized gradient approximation (GGA) functional of PW91 for the exchange-correlation. The valence-core 
interaction is described by the projector-augmented wave (PAW) approach. Computations are carried out using Vienna ab-initio simulation (VASP) code [87]. To determine the optimized structure, we have used a $1 \times 1 \times 7$ (4 irreducible k-points) k-point grid within the Monkhorst-Pack (MP) scheme to sample the Brillouin zone. To check the accuracy and convergence of our results, we have also performed structural optimizations of the NW by using $1 \times 1 \times 8$ and $1 \times 1 \times 11 \mathrm{k}$-point grids within the MP scheme. The relative difference in cohesive energy by increasing the irreducible k-points from 4 to 6 is found to be $0.001 \%$ while the relative difference in band gap energy is $0.01 \%$. We consider the structure to be optimized when the force on an individual atom is $\leq 0.01 \mathrm{eV} / \AA$. The convergence criterion for the energy during the self-consistent calculation is taken to be $10^{-6} \mathrm{eV}$. The energy cut off for the plane wave basis is $280 \mathrm{eV}$. It is well known that the energy bandgap obtained from the ground state Kohn-Sham approach does not represent the actual quasiparticle gap measured in the experiment. Thus, it is important to discuss whether the many-body correlation effect, which has been found to be significant for small diameter NW, affects the $\Delta E_{g}$ obtained from GGA based DFT. It has been shown in Si-nanowire that the energy gap obtained using local density approximation (LDA) is significantly smaller than the observed value, which can be corrected by using self-energy correction within the GW approximation [116]. However, a recent Configuration Interaction (CI) based study in Si nanocrystal suggests that excited state correction method does not make notable difference as compared to the GGA based DFT [117]. In addition, numerous studies have also confirmed the usefulness of DFT in predicting the trend of energy band gap in nanowires 


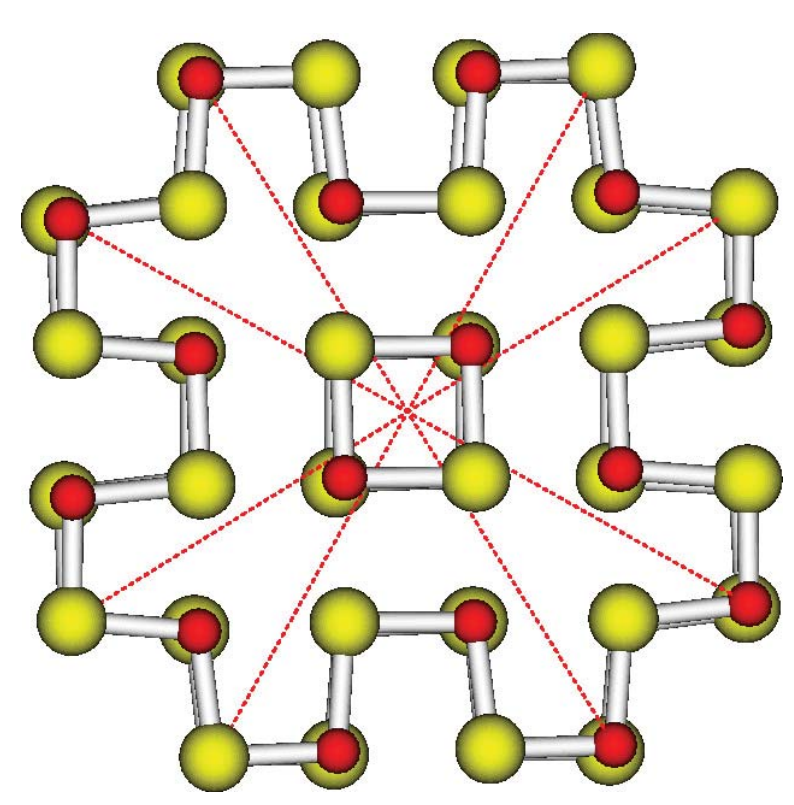

Figure 5.1: $\quad$ Structure of $\mathrm{PbS}$ nanowire of $\mathrm{d} \sim 1.98 \mathrm{~nm}$ : Cross sectional view of one unit cell in (200) direction. S, dark gray (red); Pb, light gray (golden). Reprinted figure with permission from Subhasish Mandal and Ranjit Pati, Chem. Phys. Lett. 479, 244 (2009). (C) The Elsevier.

$[118,119,120]$. Thus, the trends in $\Delta E_{g}$ that we have observed in $\mathrm{PbS}$ nanowire is not expected to change

\subsubsection{Results and Discussions}

First, we performed energy band structure calculations for the PbS bulk structure to calibrate our computational approach. We have used $21 \times 21 \times 21 \mathrm{k}$-point grid within the MP scheme to sample the brillouin zone (BZ). The energy band diagram is summarized in Fig. 5.2. One can notice from Fig. 5.2 that the $\mathrm{PbS}$ in the bulk phase is a direct band gap semiconductor. The optimum lattice parameter is $5.98 \AA$. At the L-point the 
$\Delta E_{g}$ is found to be $0.44 \mathrm{eV}$. This is in very good agreement with the $0.41 \mathrm{eV}$ for $\Delta E_{g}$ reported from the experiment [113]. It should be noted that the use of $18 \times 18 \times 18 \mathrm{k}$-point sampling within the MP scheme to sample the brillouin zone yields a gap of $0.42 \mathrm{eV}$ at the L-point, suggesting that convergence in $\Delta E_{g}$ is achieved in our calculation with respect to the number of k-points, adding further confidence in our results. As discussed in the previous section, nanowires of different diameters are engineered along the observed (200) growth direction and optimized.The cross sectional view of the optimized unit cell structure from a representative nanowire of diameter $\sim 1.98 \mathrm{~nm}$ is shown in Fig. 5.1. First, we comment on the stability. To infer the stability of the NW, we have calculated the cohesive energy per atom $\left(E_{c}\right)$ for each NW, which is summarized in Table 5.1. One notes from Table 5.1 that the maximum difference in $E_{c}$ between the bulk and the NW is $\sim 0.1 \mathrm{eV}$; this suggests that the stability of the NWs are comparable to that of the bulk. As expected, the difference in $E_{c}$ between $\mathrm{PbS}$ nanowire and bulk $\mathrm{PbS}$ decreases as the diameter of the nanowire increases. The difference in $E_{c}$ between NW of diameter $\sim 3.64 \mathrm{~nm}$ and bulk $\mathrm{PbS}$ is only $\sim 0.04 \mathrm{eV}$. Second, $\Delta E_{g}$ (Table 5.1) is found to decrease as the diameter of the NW increases. For a NW of diameter $\sim 1.17 \mathrm{~nm}$, the energy band gap is found to be $1.524 \mathrm{eV}$, which decreases to $0.955 \mathrm{eV}$ for a NW with diameter $\sim 3.64 \mathrm{~nm}$. More importantly, we found a monotonic decrease in the energy band gap with an increase in diameter. To develop an atomic level understanding of the observed change in $\Delta E_{g}$, we analyzed the nearest neighbors' bond length between $\mathrm{Pb}$ and $\mathrm{S}$ atoms within the $\mathrm{x}-\mathrm{y}$ plane. As the diameter decreases, the average nearest neighbor distance, $l$, between $\mathrm{Pb}$ and $\mathrm{S}$ is 


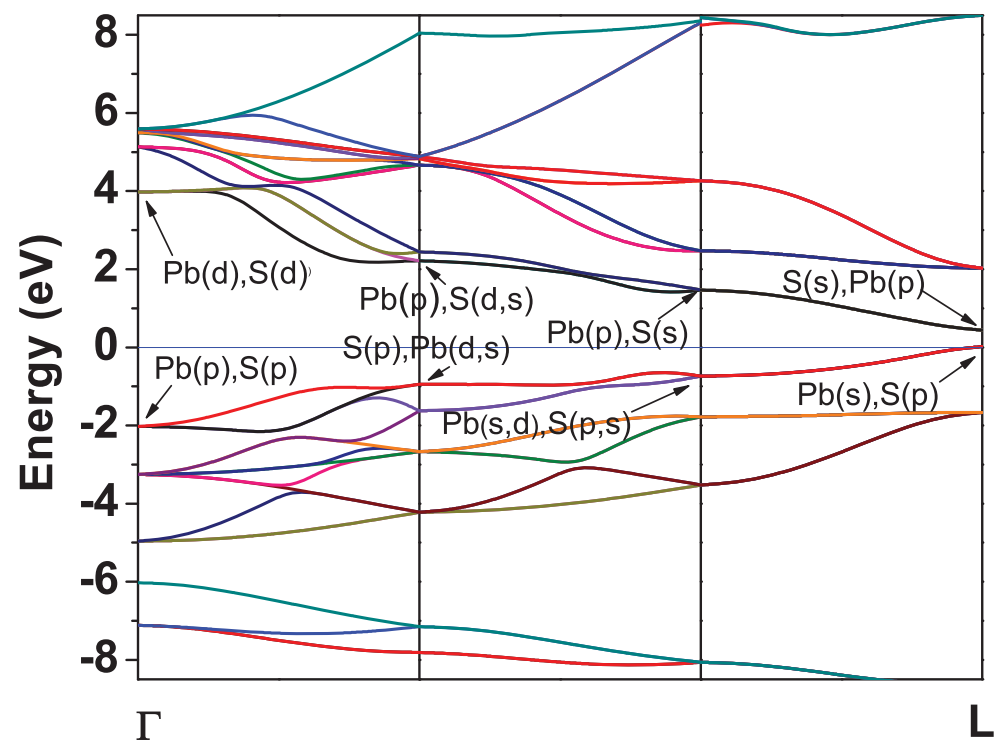

Figure 5.2: Electronic energy band structure for bulk $\mathrm{PbS}$ to calibrate our computational approach with experiment. The Fermi level lies at $E=0$. Reprinted figure with permission from Subhasish Mandal and Ranjit Pati, Chem. Phys. Lett. 479, 244 (2009). (c) The Elsevier.

found to decrease (Table 5.1), resulting in a more confined charge density. This higher degree of quantum confinement for a smaller diameter NW is resulting in an increase in its band gap.

Table 5.1

Calculated diameter (d), average $\mathrm{Pb}-\mathrm{S}$ bond length $(l), E_{c}$, and $\Delta E_{g}$ for $\mathrm{PbS}$ NW.

\begin{tabular}{llll}
\hline $\mathrm{d}(\mathrm{nm})$ & $l(\mathrm{~nm})$ & $E_{c}(\mathrm{eV})$ & $\Delta E_{g}(\mathrm{eV})$ \\
\hline 1.17 & 0.283 & 4.316 & 1.524 \\
1.98 & 0.286 & 4.356 & 1.299 \\
2.8 & 0.289 & 4.375 & 1.098 \\
3.64 & 0.295 & 4.380 & 0.955 \\
Bulk & 0.299 & 4.426 & 0.44 \\
\hline
\end{tabular}




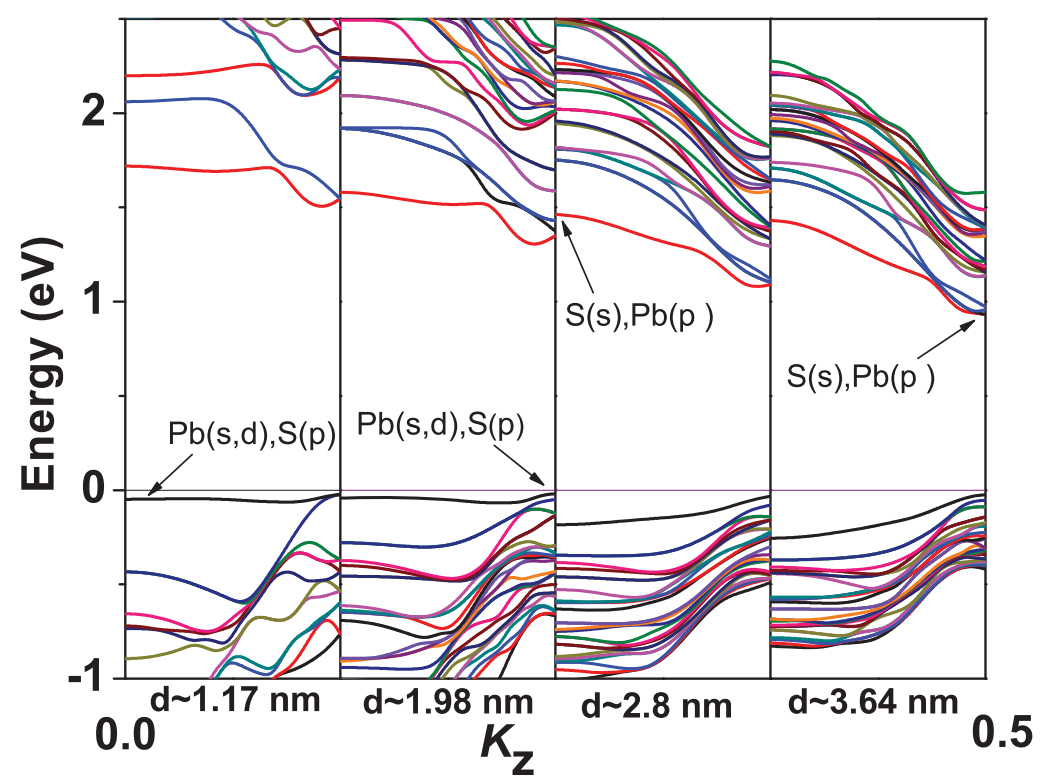

Figure 5.3: Electronic energy band structure for $\mathrm{PbS}$ nanowires with different diameters. The Fermi level lies at $E=0$. Reprinted figure with permission from Subhasish Mandal and Ranjit Pati, Chem. Phys. Lett. 479, 244 (2009). (c) The Elsevier.

To show the diameter dependence of the energy bands, we have plotted the Kohn-Sham energy bands for all four NWs in Fig. 5.3. For larger diameter NW, the minimum energy point in the conduction band (CBM) and the maximum energy point in the valence band (VBM) appear at the same $\mathrm{k}$ point. This confirms their direct band gap property as observed in bulk PbS [115]. But when the diameter decreases, the CBM shifts towards the $\Gamma$-point, resulting in an indirect band gap behavior. Furthermore, as expected, the degeneracy in the energy bands is lifted as the diameter decreases. The conduction band is found to be more sensitive to the decrease in diameter. It should be noted that, very recently, a similar diameter dependence feature is found in tapered silicon nanowires [121, 119]. The 
wavefunction for the conduction band near the $\mathrm{X}$-point is found to be $\mathrm{S}(s)-\mathrm{Pb}(p)$ hybridized for the NW with larger diameter. However, as the diameter decreases, the virtual $3 d$ orbital of the $\mathrm{S}$ contributes to the conduction band resulting in an indirect band gap feature.

\subsubsection{Strain Induced Phase Transition}

Now we turn our discussions toward the strain induced phase transition of the nanowire. $\mathrm{PbS}$ nanowires of diameter $\sim 1.2 \mathrm{~nm}$ grown in Na-4 Mica channels have been found to exhibit semiconducting to metallic phase transition at $\sim 300 \mathrm{~K}[105]$. The thermal expansion mismatch at the $\mathrm{PbS}$ and $\mathrm{Na}-4$ Mica interface, producing $\sim 3 \mathrm{GPa}$ pressure on the nanowire, has been suggested as the cause for this phase transition. To replicate the effect of radial pressure on the nanowire, we have applied uniform compressive radial strain on a representative NW of diameter $\sim 1.98 \mathrm{~nm}$. The NW is allowed to relax in the (200)

growth direction under radial strain. The $\%$ radial strain, $\zeta$, is defined as: $\zeta=\frac{100 \Delta r}{r_{0}}$, where $\Delta r=r_{0}-r ; r$ and $r_{0}$ are the radii of the nanowire with and without the radial strain respectively. The energy band diagrams under different $\zeta$ are presented in Fig. 5.4. One can clearly notice the semiconducting to metallic phase transition at $\zeta=12 \%$. The conduction band, which has a contribution from the excited $3 d$ orbital of $\mathrm{S}$, is found to be more sensitive to the compressive radial strain (CS) with the CBM shifting towards and eventually crossing the Fermi energy with increasing CS. The contribution from the $3 d$ orbital of $\mathrm{S}$ at the $\mathrm{CBM}$ develops a bonding character in the part of the $\mathrm{CB}$ wavefunction 


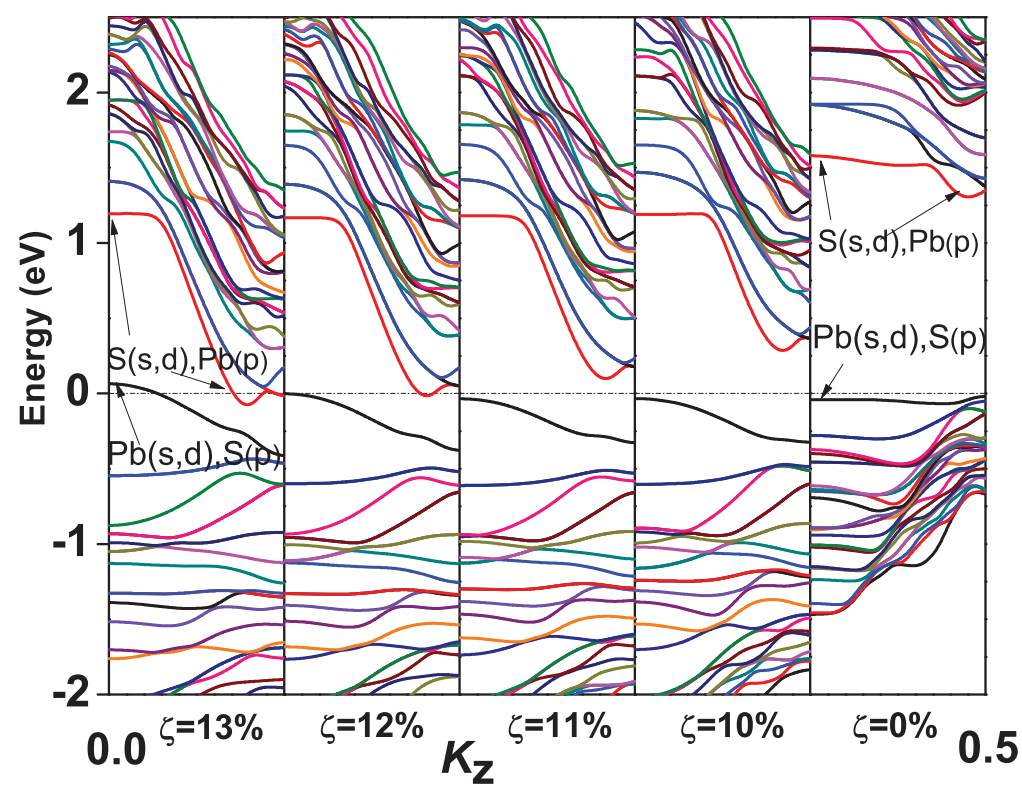

Figure 5.4: (a) Electronic energy band structures for $\mathrm{PbS}$ nanowire with $\mathrm{d} \sim 1.98 \mathrm{~nm}$ for different $\zeta$ s. The Fermi level lies at E=0. Reprinted figure with permission from Subhasish Mandal and Ranjit Pati, Chem. Phys. Lett. 479, 244 (2009).(C) The Elsevier.

resulting in the reduction of its energy under CS. A similar effect of shifting the conduction band under compressive strain is noted in bulk $\mathrm{Si}$ [119]. The valence band, which is almost dispersionless, develops $\sim 0.4 \mathrm{eV}$ dispersion width under compressive radial strain. For the NW with $\mathrm{d} \sim 1.17 \mathrm{~nm}$, the semiconducting to metallic phase transition occurs at about $\zeta=13 \%$. To calculate the amount of pressure required for the phase transition, we have plotted the relative deformation potential energy $(E)$ as a function of $\zeta$ in Fig. 5.5 for the NW of diameter $\sim 1.98 \mathrm{~nm}$. The excellent parabolic behavior of $E$ from Fig. 5.5 allows us to use Hooke's law to determine the coefficient for radial stiffness, $S_{r}$, which is defined as: $S_{r}=\frac{\delta^{2} E}{\delta r^{2}} \frac{1}{2 \pi z_{0}} \cdot z_{0}$ is the length of the unit cell $(0.6 \mathrm{~nm})$ at $\zeta=0$. From the second derivative 
of the deformation potential $E$, we have calculated the $\frac{\delta^{2} E}{\delta \zeta^{2}}$ as $1.9692 \mathrm{eV}$. Subsequently, the $\frac{\delta^{2} E}{\delta r^{2}}$ is obtained by using the undeformed radius $r_{0}=0.988 \mathrm{~nm}$. The $S_{r}$ is found to be 857 GPa. To calculate the amount of pressure $(P)$ required for the semiconducting to metallic phase transition $\left(\zeta=12 \%\right.$ ), we follow the simple relation $P=S_{r} \frac{\Delta r}{r}$ that yields $102.8 \mathrm{GPa}$ for the value of $P$. For the NW of $\mathrm{d} \sim 1.17 \mathrm{~nm}$, using the same $S_{r}$, the $P$ is found to be 111.4 GPa. These values are found to be higher than the reported pressure ( $\sim 3 \mathrm{GPa})$ for the phase transition estimated using the bulk modulus ( $127 \mathrm{GPa})$ of $\mathrm{PbS}[105]$. The substantial difference between experiment and theory can be attributed to the lower bulk modulus value [105] used to estimate $P$ in the experiment. The use of the bulk modulus $\sim 127 \mathrm{GPa}$ for $S_{r}[105]$ in our calculation would yield $P=15.2 \mathrm{GPa}$ for $\mathrm{d} \sim 1.98 \mathrm{~nm}$, and $P=16.5 \mathrm{GPa}$ for $\mathrm{d} \sim 1.17 \mathrm{~nm}$. These are about five times greater than the experimentally obtained $P$. In addition, the calculation reported here is for uniform, pristine, and defect free $\mathrm{PbS}$ NWs, unlike the experimental case, where the $\mathrm{PbS}$ NWs are grown within the Na-4 Mica channels in different directions.

\subsection{Gate Field Induced Switching of Current in One}

\section{Dimensional Nanowire Junction}

Now we will discuss the charge transport in $\mathrm{PbS}$ nanowire junction and investigate the gate field induced switching of current. Field effect transistors (FET), whose main 


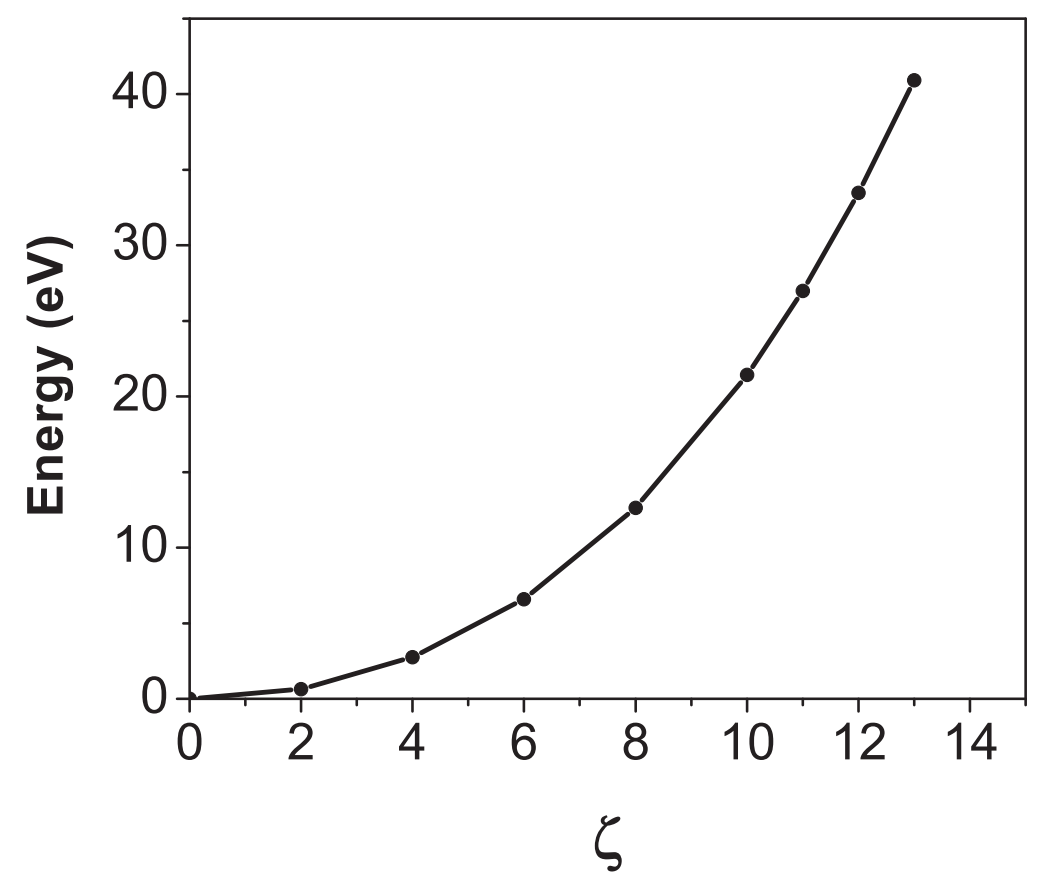

Figure 5.5: The variation of the relative deformation potential energy with $\zeta$. Reprinted figure with permission from Subhasish Mandal and Ranjit Pati, Chem. Phys. Lett. 479, 244 (2009). (c) The Elsevier.

function is to switch source-drain current upon the application of gate field, have been fabricated from semiconducting nano-wires (NW) and nano-crystal arrays [92, 93, 122]; the switching speed for such devices, in some instances, have been found to surpass that of the conventional semiconductor-FET. Particularly, IV-VI $[123,124,125,122]$ semi conducting nano-wires based FET devices have been shown to have huge conductivity and high current gain, which are key requisites for an integrated circuit. The controlled synthesis of these NWs with diameter ranging from $1.2 \mathrm{~nm}$ to $10 \mathrm{~nm}$ have been reported $[104,105,126]$. Despite the rapid development on the experimental side, which provides 
an ample opportunity for theorist to test their models, only nominal theoretical efforts are made to understand the quantum phenomenon that dictates current modulation in such a nanowire junction (particularly NW of few nm dimension); thus far, no unswerving interpretation exists. Since the dimension of the channel is in the nanoscale regime, the electronic property and/or the field effect switching mechanism is expected to be different than that of the conventional FET. Here, we present a first principles quantum transport study in a strongly coupled, single $\mathrm{PbS}$ nanowire (PbSNW) junction (Fig. 5.6) to unravel the mechanism responsible for the gate field induced switching of current. we have used the same gold electrode to form the nanowire-lead junction as used in the experiment $[123,124,125,127]$. Particularly, here we try to answer several fundamental questions: How does the gate field affect the intrinsic electronic structure of the nanowire? Can we control the number of participating orbitals of the NW-channel via gate field? Which are the orbitals that contribute to the conductance? Can we manipulate the shape of the orbital via gating? and finally, is there a universal model that would explain the observed gate field induced switching not only in PbSNW but also in other lead-chalcogenide nanowire?

\subsubsection{Modeling the device}

As discussed in Chapter 3 and Chapter 4, we have adapted the same single particle Green's function approach to investigate the gate field induced switching of current in a $\mathrm{PbS}$ nanowire junction $(\mathrm{PbSNW})$. The scattering region is comprised of a finite $\mathrm{PbSNW}$ of 


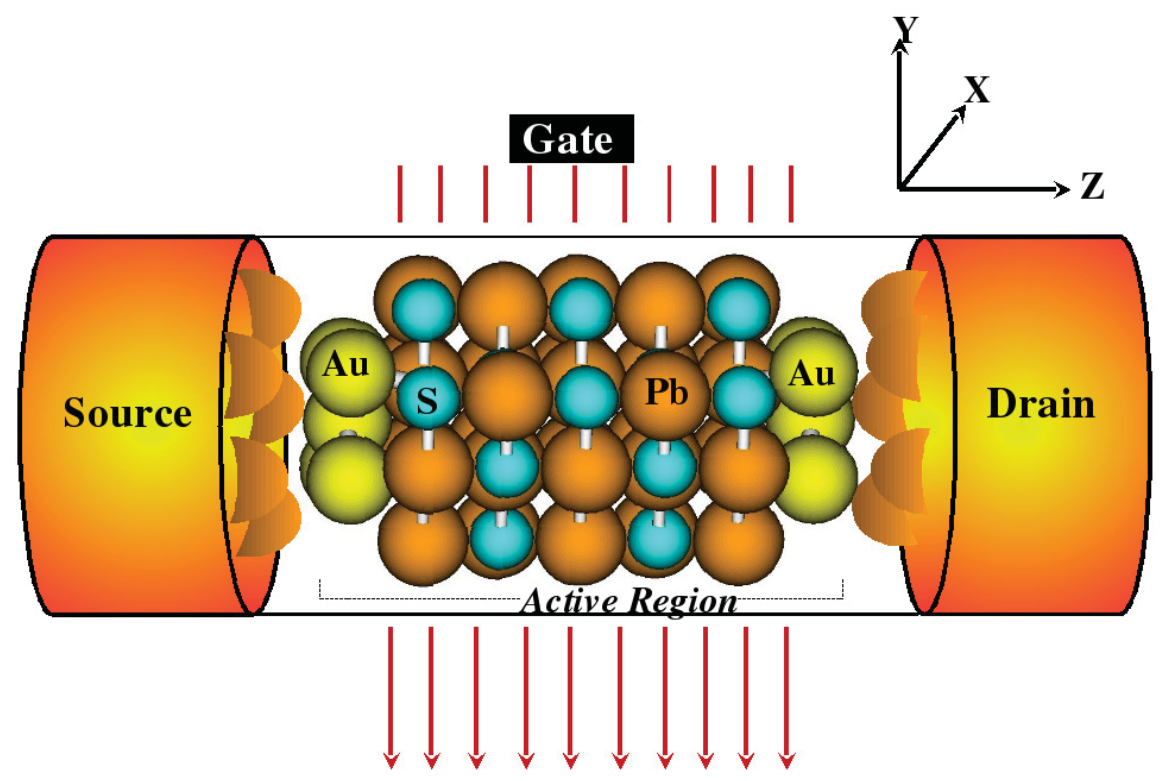

Figure 5.6: Schematic representation of a $\mathrm{PbS}$ nanowire junction; solid arrows show the direction of the applied gate field. Reprinted figure with permission from Subhasish Mandal and Ranjit Pati, Phys. Rev. B. 84 115306 (2011). (c) The American Physical Society.

length $\sim 1.2 \mathrm{~nm}$ and diameter $\sim 1.17 \mathrm{~nm}$; the second part is part of the lead that is strongly coupled to the NW and is represented only by a finite number of gold atoms (five gold atoms on each side), and the third part is the unperturbed electrode part which is assumed to retain the bulk behavior of gold. The atomic level structural details for the finite part of the PbSNW is taken from the optimized structure of an infinite NW, grown in the observed (100) direction. The later structure having six $\mathrm{Pb}$ and six $\mathrm{S}$ atoms in each layer along the growth direction with a lattice parameter of $6 \AA$ was calculated using the periodic DFT [115]. Only a five layer NW-structure along the growth direction is considered to build the symmetric junction with the lead (Fig. 5.6). The atomic composition of the lead is taken from the $\mathrm{Au}(100)$ surface to avoid the lattice mismatch at the NW-lead interface. To realize 
a strongly coupled junction between the NW and the lead, the interfacial distance is varied to determine the optimum distance $(2.80 \AA)$ where the repulsive interaction is minimum.

The difference in modeling from the earlier cases is the inclusion of gate field together with the source-drain field in our calculations. In the present case, the Hamiltonian of the active region $\left(\mathrm{NW}+\right.$ finite lead) of the device is expressed as: $H\left(\vec{\varepsilon}_{d}\right)=H(0)+\vec{\varepsilon}_{d}$. $\sum_{i} \vec{r}_{i}+\vec{\varepsilon}_{g} \cdot \sum_{i} \vec{r}_{i}$, where $H(0)$ is the Hamiltonian in the absence of electric field; $\vec{\varepsilon}_{d}$ is the applied dipole electric field along the axis parallel to the direction of current flow (z-axis), and $\vec{r}_{i}$ is the coordinate of the $i^{t h}$ electron; charging effect on the NW is considered by including a finite part of the lead. The self-consistent inclusion of dipole interaction term permits us to include both first and higher order Stark effects, which is also evident from the comparison of total energy in the active region for different $\vec{\varepsilon}_{d}$; a non-linear change in energy by increasing the strength of the $\vec{\varepsilon}_{d}$ confirms the inclusion of higher order effects. This intrinsic charge imbalance between the two leads is also reflected from the potential profile summarized in Fig. 5.7. The relative electrostatic potential (REP) in Fig. 5.7 is calculated by subtracting the average potential at each atomic site in a layer at the EB condition from that at the NEB condition. A linear drop in the REP value along the wire axis is noted. The magnitude of the potential drop at both the junctions are equal confirming the NW-junction to be symmetric. A non linear change in the REP values with different $\vec{\varepsilon}_{d}$ elucidates the nonlinear response of the field. The REP values at the left and right $\mathrm{Au} l e a d$, which are assumed to be at same potential with semi-infinite electrodes on left and right respectively, are used to calculate $V_{L}$ and $V_{R}$. The electro chemical potentials 
at the two semi-infinite contacts are obtained as: $\mu_{L, R}=V_{L, R} \mp k_{B} T[27,28]$. A small thermal smearing term $\left(\sim k_{B} T\right)$ in $\mu_{L}$ and $\mu_{R}$ takes into account the electronic temperature at the contact in the NEB condition; the potential difference between source and drain $\left(V_{s d}\right)$ is then obtained from the difference of $\mu_{L}$ and $\mu_{R}$. In order to simulate the effect of electrostatic gating, we have included an additional dipole interaction term $\left(\vec{\varepsilon}_{g} \cdot \sum_{i} \vec{r}(i)\right)$ in the Hamiltonian; the dipole field $\vec{\varepsilon}_{g}$ is applied along the direction perpendicular to the channel axis and is referred to as the transverse gate field in this article. In our calculation, we have used a posteriori hybrid density functional method (B3LYP) that includes a portion of the exact Hartree-Fock exchange. The LANL2DZ effective core potential basis set, which includes scalar relativistic effect, is used to describe the $\mathrm{Pb}$ and $\mathrm{Au}$ atom in the device; a triple zeta augmented by polarization function $\left(6-311 \mathrm{G}^{*}\right)$ basis set is used for the S. Subsequently, we recourse to implicit bias-dependent Green's function approach $[10,28]$ to couple the finite NW to the infinite electrode via the finite self-energy functions; coherent, single particle scattering formalism is used to calculate the electronic current.

\subsubsection{Current-Voltage Characteristics}

The calculated current-voltage $\left(I_{s d}-V_{s d}\right)$ characteristic for $\mathrm{PbSNW}$ as a function of $\varepsilon_{g}$ is summarized in Fig. 5.8. The strength of $\varepsilon_{g}$ is mapped to the gate potential, $V_{g}$, by calculating the potential drop self-consistently between the terminal atomic layers of the NW along the direction of gate field. For $V_{g}=0 \mathrm{~V}$, a steady increase in current $\left(I_{s d}\right)$ is noted 


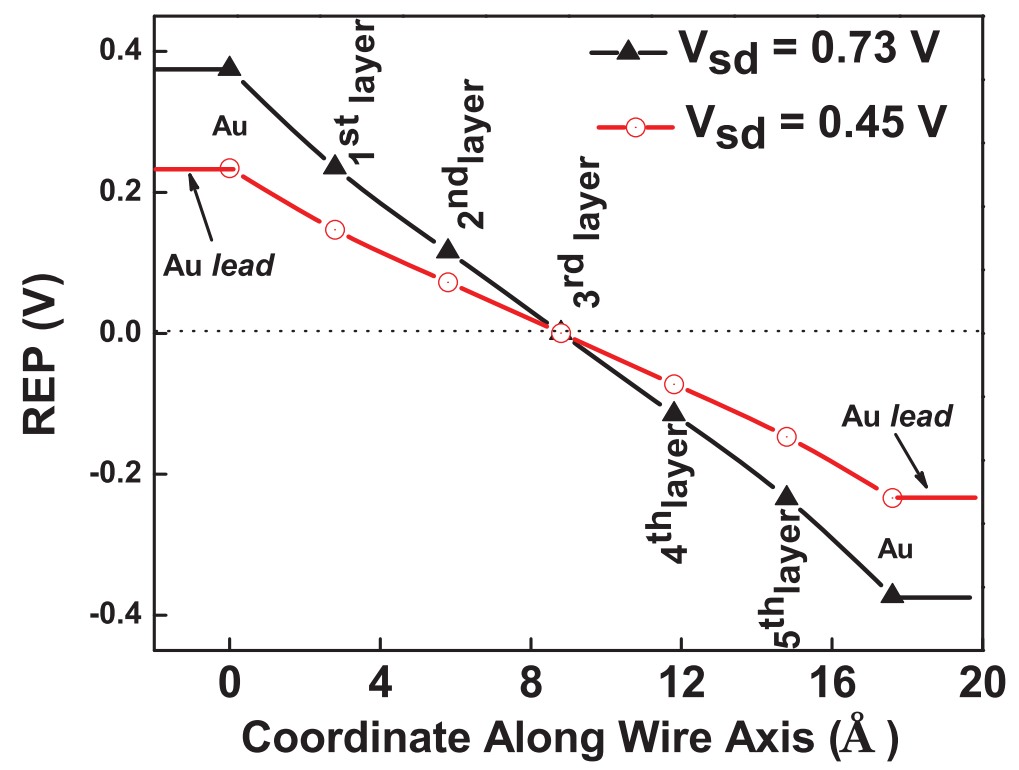

Figure 5.7: Electrostatic potential profile of the NW junction in the absence of gate bias for two different $V_{s d}$. Reprinted figure with permission from Subhasish Mandal and Ranjit Pati, Phys. Rev. B. 84115306 (2011). (C) The American Physical Society

with the increase of $V_{s d}$. Changing the $V_{g}$ from $0 \mathrm{~V}$ to $-3.7 \mathrm{~V}$, though an analogous linear increase in $I_{s d}$ as a function of $V_{s d}$ is observed, the magnitude of the current is found to be higher $\left(\sim 1.5\right.$ times at $\left.V_{s d} \sim 0.7 \mathrm{~V}\right)$ for $V_{g}=-3.7 \mathrm{~V}$. A further change of $V_{g}$ from $-3.7 \mathrm{~V}$ to $-5.6 \mathrm{~V}$ reveals a considerable increase in $I_{s d}$. Changing the $V_{g}$ from $-5.6 \mathrm{~V}$ to $-7.6 \mathrm{~V}$, a non-linear feature in $I_{s d}$ is noted. The current at $V_{s d}=0.74$ is found to be 2.32 times higher for $V_{g}=-5.6 \mathrm{~V}$ than that for $V_{g}=-3.7 \mathrm{~V}$; increasing the negative gate potential from $-5.6 \mathrm{~V}$ to $-7.6 \mathrm{~V}, 1.53$ times higher current is found at $V_{s d} \sim 0.7 \mathrm{~V}$. Thus comparing between $V_{g}=0 \mathrm{~V}$ and $V_{g}=-7.6 \mathrm{~V}, \sim 5$ times increase in $I_{s d}$ is found at $V_{s d} \sim 0.7 \mathrm{~V}$. To illustrate this behavior, we have plotted $I_{s d}$ as function of $V_{g}$ in the inset of Fig. 5.8; a fixed $V_{s d}$ is used. First a 


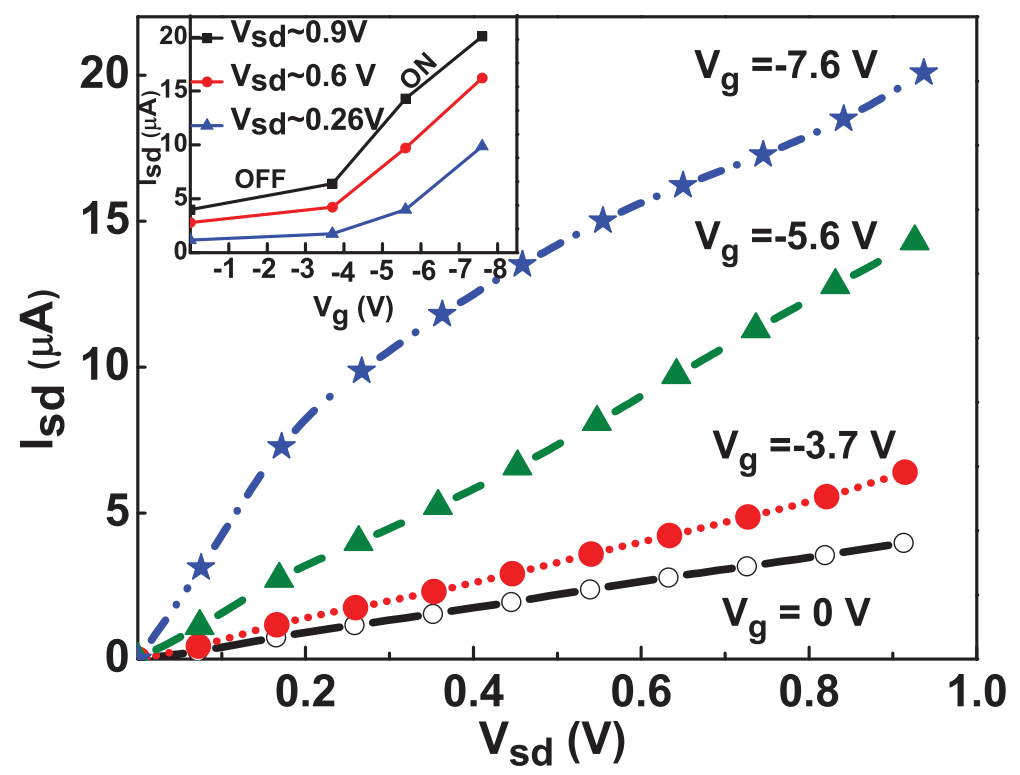

Figure 5.8: Current-Voltage characteristics with different $V_{g}$ for $\mathrm{PbS}$ nanowire junctions. The insets show $I_{s d}-V_{g}$ plot for different $V_{s d}$. Reprinted figure with permission from Subhasish Mandal and Ranjit Pati, Phys. Rev. B. 84115306 (2011). (c) The American Physical Society

slow increase in current (OFF state) is noted till the value of $V_{g}$ reaches a threshold value $\left(V_{g}^{t h}\right)$ of -3.7 V. After $V_{g}=-3.7 \mathrm{~V}$, a steep increase in current (ON state) is observed with the increase of gate potential resulting in a large change in the slope of $I_{s d}-V_{g}$.

The calculated ON/OFF current ratio value is found to be 6.28 at $V_{s d}$ of $0.54 \mathrm{~V}$ between $V_{g}=0 \mathrm{~V}$ and $V_{g}=-7.6 \mathrm{~V}$. A similar switching feature is also found in a PbSe NW junction. To construct the device and to investigate the gate field induced switching phenomena in $\mathrm{PbSe}$ nanowire junction, we have adopted the same approach as we discussed for $\mathrm{PbS}$ nanwire. The calculated current-voltage $\left(I_{s d}-V_{s d}\right)$ characteristic for PbSe nanowire as a function of 


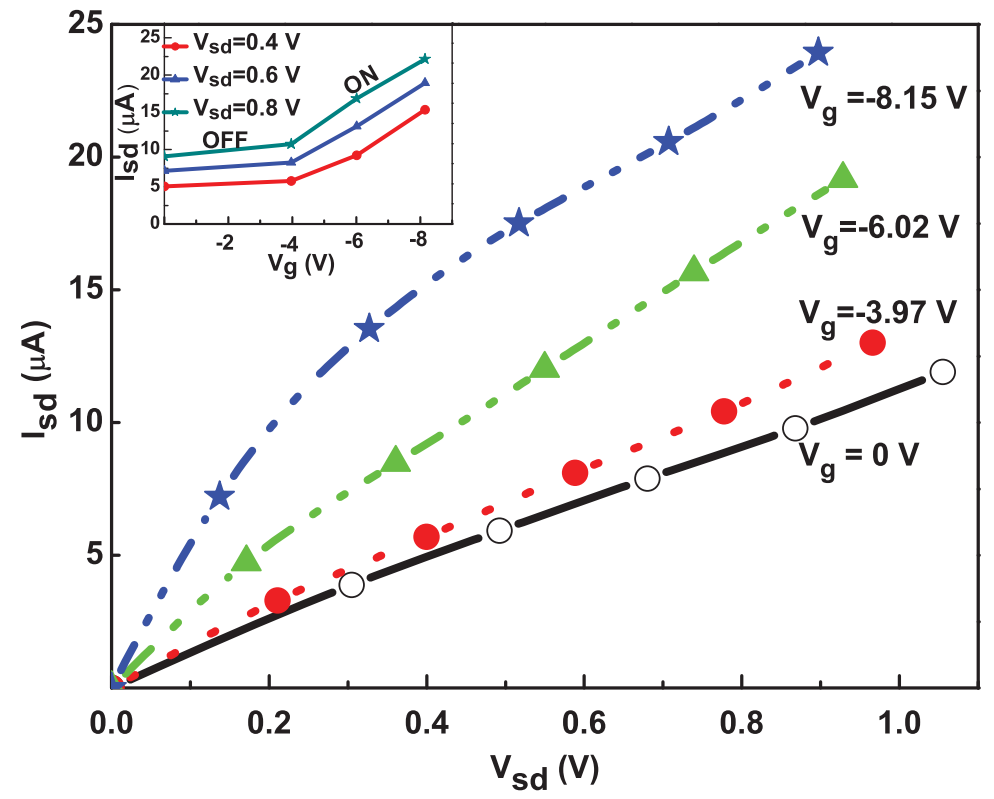

Figure 5.9: Current-Voltage characteristics with different $V_{g}$ for $\mathrm{PbSe}$ nanowire junctions. The insets show $I_{s d}-V_{g}$ plot for different $V_{s d}$. Reprinted figure with permission from Subhasish Mandal and Ranjit Pati, Phys. Rev. B. 84115306 (2011). (c) The American Physical Society

$V_{g}$ is plotted in Fig. 5.9.

\subsubsection{Which Density Functional to Choose?}

It should be noted that the ON/OFF current ratio of $\sim 3.75$ between $V_{g}=0 \mathrm{~V}$ and $V_{g}=-8$ $\mathrm{V}$ at $V_{s d}$ of $0.5 \mathrm{~V}$ is observed in a recent experiment, where a single $\mathrm{PbS}$ nanowire is used as a channel. The magnitude of $I_{s d}$ reported in the experiment is in the nA range in contrast to the $\sim \mu \mathrm{A}$ current observed in our calculations. Several reasons could be 
attributed to the observed differences in $I_{s d}$. First, in the experiment the channel length and diameter were $10^{3}$ and $150 \mathrm{~nm}$ respectively, where the diffusive transport could be the prevalent mechanism. In contrast, we have considered the channel length and diameter to be $1.2 \mathrm{~nm}$ and $1.17 \mathrm{~nm}$ for practical purposes. Considering an approximate exponential decay in current with the length $(l)$ for the nanowire used in the experiment ( $\sim e^{-\beta l} ; \beta$-decay constant), we would expect the measured current to be of the order of $\mu \mathrm{A}$ for a few nm channel length, which has also been reported experimentally in single PbSe semiconducting nanowire junction [124]. In addition, we have considered an ideal, defect-free nanowire junction. The magnitude of higher current observed in our calculation is also not surprising considering the use of static exchange and correlation potential instead of the true dynamical exchange correlation corrected potential $[74,75,72,130]$. However, the consistent increase of calculated current upon increasing negative gate bias as observed in the experiments $[124,127]$ reaffirms on the switching phenomenon replicated here.

To examine, whether the increase in magnitude of current is due to the use of an implicit orbital dependent B3LYP functional approach, we have calculated the current in the same device geometry using different exchange-correlation functional; same Gaussian basis sets are used for all the calculations. Our results are summarized in Fig. 5.10. Though all different functionals (SVWN, PW91PW91, PW91LYP) [65] yield similar current-voltage characteristic, the magnitude of the current is found to be much higher $(\sim 3$ times at a $V_{s d}$ of $\sim 0.7 \mathrm{~V}$ ) than that obtained with the B3LYP approach. An atomic self interaction corrected DFT scheme yielding a lower conductance than the conventional DFT approach 


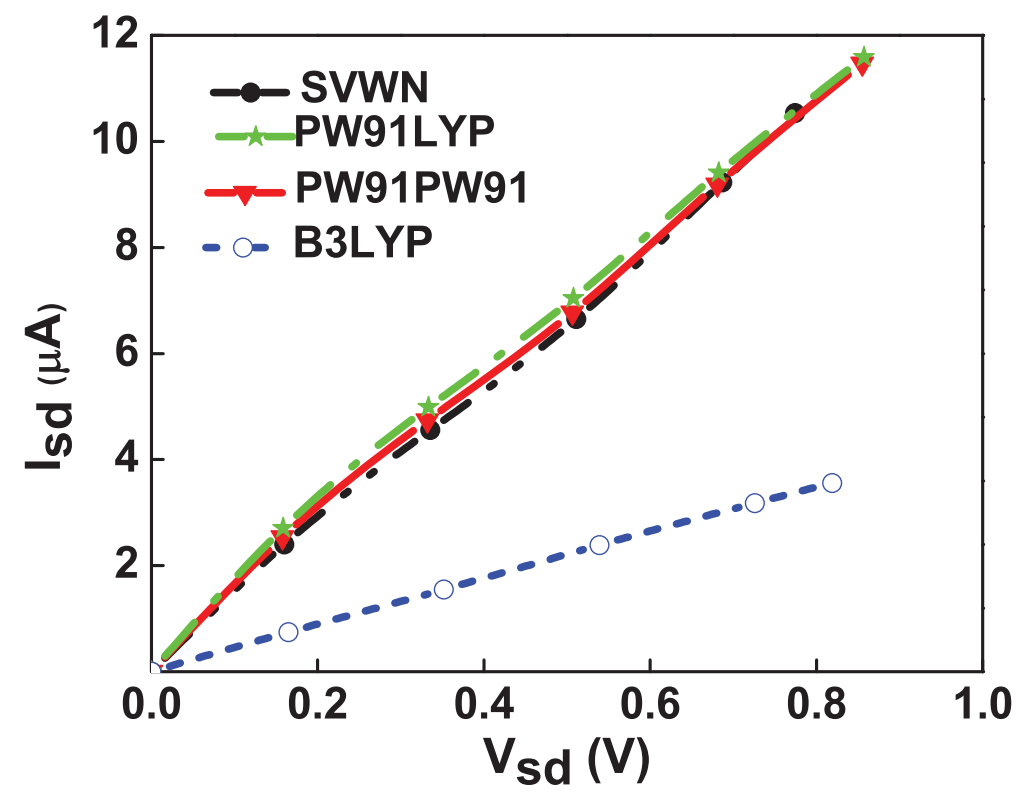

Figure 5.10: Current-Voltage characteristics of PbSNW for different exchange-correlation functionals at $V_{g}=0 \mathrm{~V}$. Reprinted figure with permission from Subhasish Mandal and Ranjit Pati, Phys. Rev. B. 84 115306 (2011). (c) The American Physical Society.

has been reported in a molecular junction [128]. The inclusion of part of the exact exchange from the Hartree-Fock formalism in our posteriori B3LYP approach corrects partly the self-interaction error that occurs in the conventional density functional method; it represents a substantial improvement in the right direction as evident from the $V_{s d}-I_{s d}$ curve (Fig. 5.10). 


\subsubsection{Bias Dependent Transmission}

To investigate the intriguing features in gate field induced current and to understand the origin of the field effect behavior in PbSNW, we have calculated the bias dependent transmission function as a function of injection energy $(E)$ for different $V_{g}$ (Fig. 5.11). For brevity, we have only considered $V_{s d} \sim 0.76 \mathrm{~V}$. First, the increase of area under the transmission curve within the chemical potential window (CPW) with the increase of negative gate bias confirms the observed increase of $I_{s d}$ with $V_{g}$ (Fig. 5.8); the non-linear increase in area explains the change of slope in $I_{s d^{-}} V_{g}$ plot presented in the inset of Fig. 5.8. Analysis of eigenvalues of Hamiltonian for the NW reveals unoccupied levels (shown in Fig. 5.11) contributes to the conduction. Increasing the $V_{g}$, the participating unoccupied eigen-channel shifts in the direction of Fermi energy. For $V_{g}=0 \mathrm{~V}$, only L0 level contributes to the $T(E, V)$ within the CPW. As $V_{g}$ increases more unoccupied levels move into the $\mathrm{CPW}$, resulting in an increase in the density of states within the CPW. To quantify the response of the gate field, we have plotted the Stark shift ( $\varepsilon_{g}^{i}-\varepsilon_{0}^{i}$; $i$-corresponds to different unoccupied levels, $\varepsilon_{g}$ and $\varepsilon_{0}$ are respectively the orbital energy in the presence and absence of gate field) as a function of $V_{g}$ for different participating unoccupied levels in Fig. 5.12. A significant Stark shift has been observed. Different levels exhibit different shift, particularly at higher $V_{g}$. A closer examination indicates a non linear increase of Stark shift $\left(\Sigma_{i} \alpha_{i} \varepsilon_{i}+\frac{1}{2} \Sigma_{i, j} \beta_{i j} \varepsilon_{i} \varepsilon_{j}+\ldots\right)$ with the increase of $V_{g}$. 


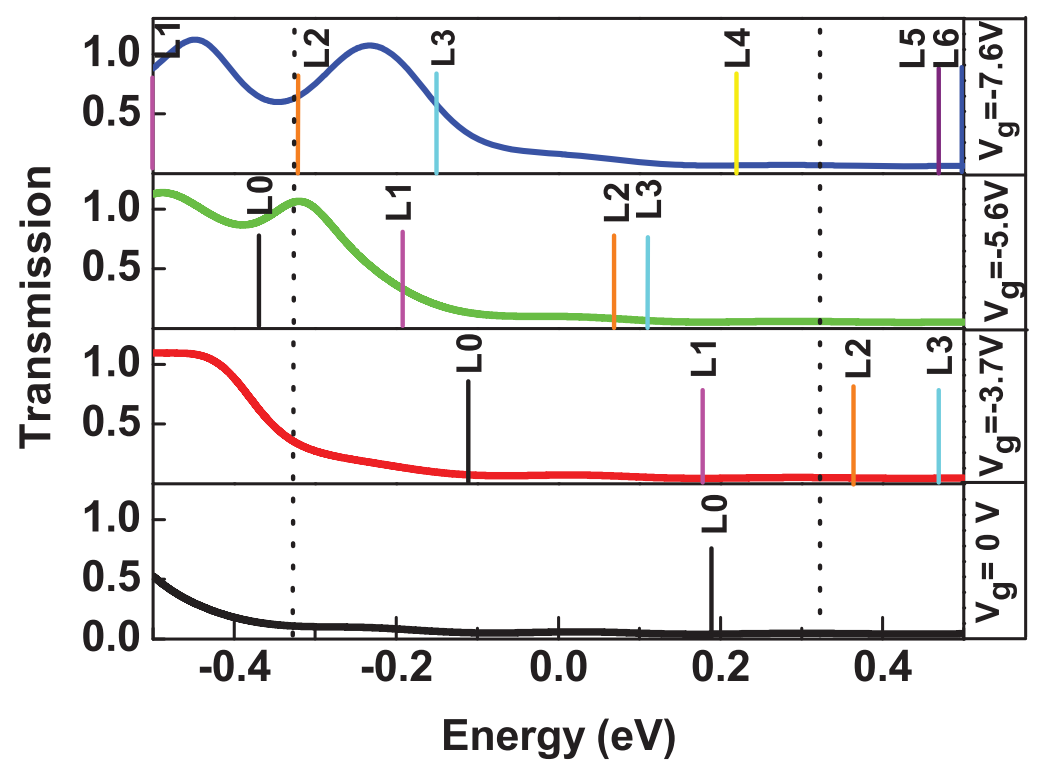

Figure 5.11: Bias dependent transmission function as a function of injection energy for different gate bias at $V_{s d} \sim 0.6 \mathrm{~V}$. The Fermi energy is set to zero in the energy scale; dotted lines represent the chemical potential window. Notation: L0, L1, L2, L3, and L4 refer to LUMO, LUMO+1, $\mathrm{LUMO}+2$, $\mathrm{LUMO}+3$, and $\mathrm{LUMO}+4$. Reprinted figure with permission from Subhasish Mandal and Ranjit Pati, Phys. Rev. B. 84115306 (2011). (c) The American Physical Society.

\subsubsection{Orbital Analysis}

Next, the natural question to ask is: How does the participating orbitals evolve with the gate bias? Does it have any correlation with the observed increase in transmission in Fig. 5.11? To answer these subtle questions, we have analyzed participating MO coefficients in the presence and absence of gate field. As expected, for $V_{g}=0 \mathrm{~V}$, the MOs are symmetric along the direction perpendicular to the wire axis (negative $\mathrm{Y}$ axis). Increasing the gate 


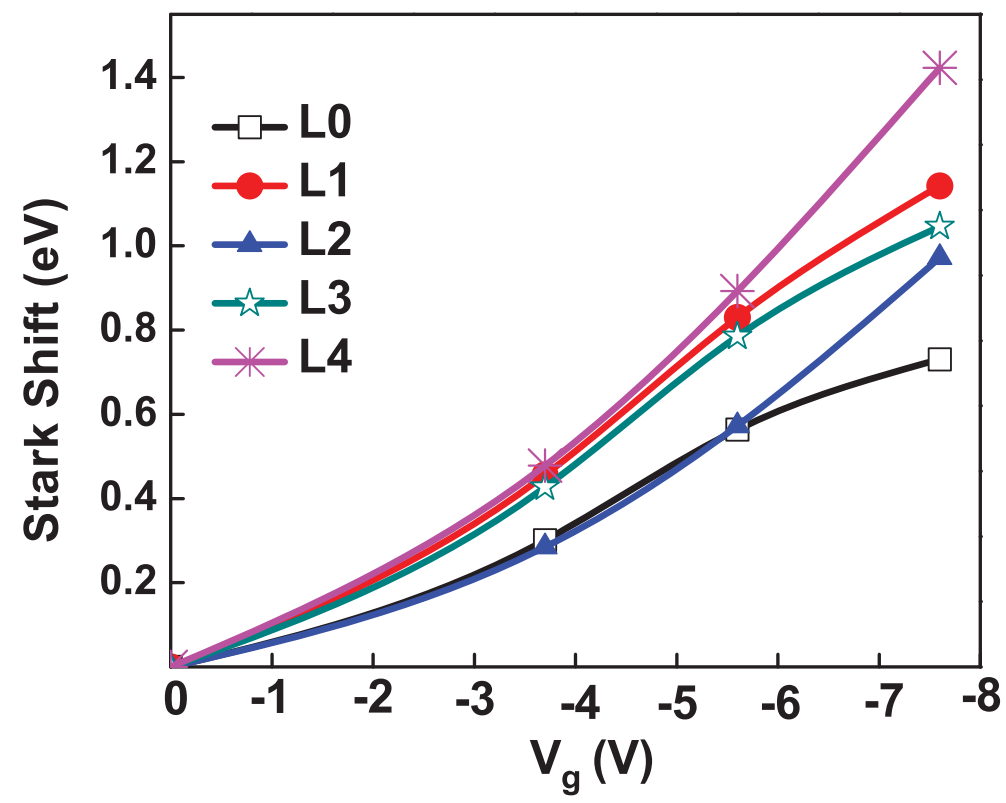

Figure 5.12: Stark shift as a function of gate bias. Notation: L0, L1, L2, L3, and L4 refer to LUMO, LUMO+1, LUMO+2, LUMO+3, and LUMO+4. A fixed $V_{s d}$ of $\sim 0.6 \mathrm{~V}$ is used. Reprinted figure with permission from Subhasish Mandal and Ranjit Pati, Phys. Rev. B. 84115306 (2011). (c) The American Physical Society.

bias to $-7.6 \mathrm{~V}$, the symmetry of the wavefunction breaks along the negative $\mathrm{Y}$ axis; the participating MOs localize in the same direction resulting the observed Stark shift (Fig. 5.12). A close inspection of the MO coefficients reveals that $S$ atoms in the participating MO have only s-components in the absence of gate bias. Increasing the gate bias beyond the threshold value of $-3.7 \mathrm{~V}, p$-components develop at the $\mathrm{S}$ atoms due to the strong gate field induced orbital mixing (Fig. 5.13). It should be noted that the $\mathrm{Pb}$ atom, which has p-component prior to the application of gate field, does not exhibit such orbital evolution. The $p$-components at the $\mathrm{S}$ atoms for the higher gate bias mediates inter-layer orbital 


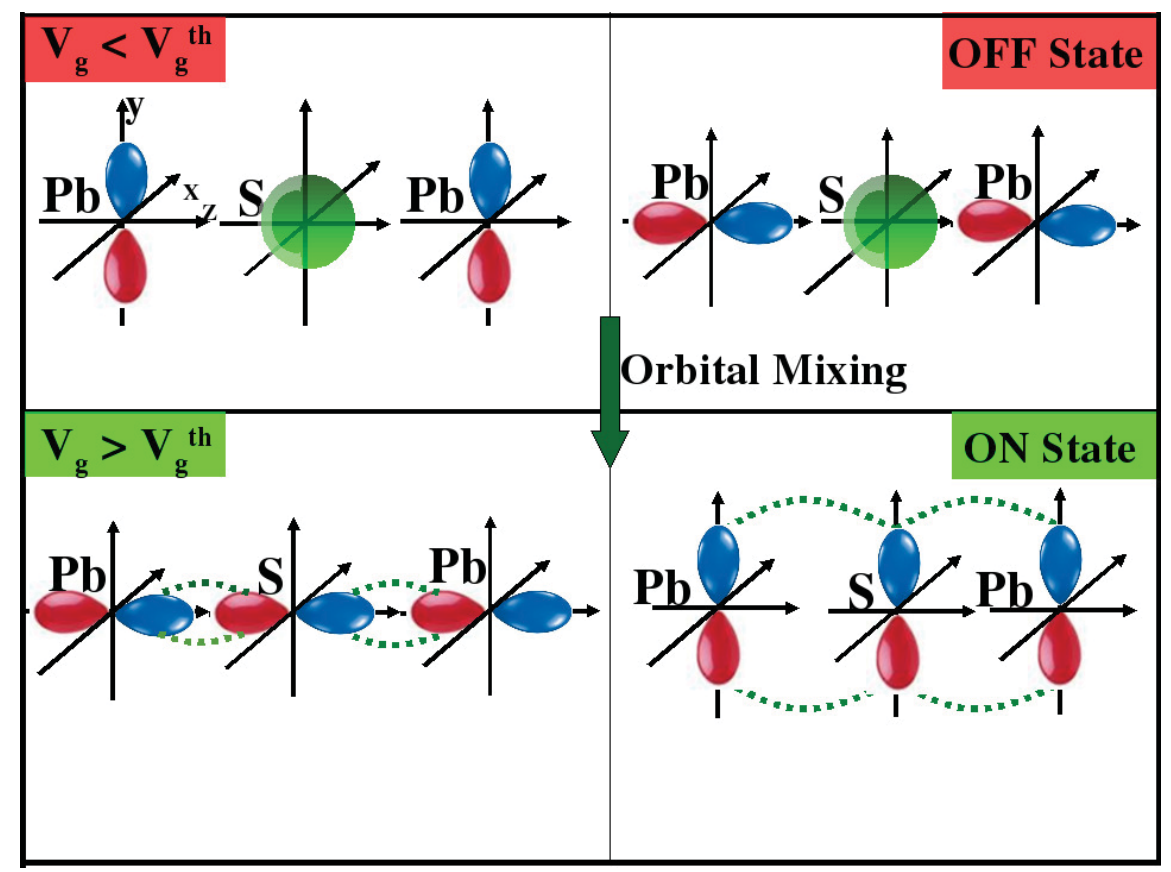

Figure 5.13: Schematic representation of orbital controlled mechanism for the PbSNW FET. In the ON state $\left(V_{g}>V_{g}^{t h}\right)$, orbital mixing produces $p$-component at the $\mathrm{S}$ atoms resulting in an inter-layer orbital interaction along the channel (z) axis. The S-atom has only s-component in the OFF state $\left(V_{g}<V_{g}^{t h}\right)$. Reprinted figure with permission from Subhasish Mandal and Ranjit Pati, Phys. Rev. B. 84115306 (2011). (C) The American Physical Society.

interaction - allowing electron to delocalize along the channel axis (Fig. 5.13). This explains unambiguously the origin of switching in conductance value observed in Fig. 5.8. It is worth mentioning that very recently orbital gating has been observed in molecular junctions [3]. 


\subsection{Conclusions}

In summary, using the density functional approach we have probed for the first time the variation of energy band gap in $\mathrm{PbS}$ nanowire with its diameter. we was able to tune the $\Delta E_{g}$ of the $\mathrm{PbS}$ nanowire from $0.955 \mathrm{eV}$ to $1.524 \mathrm{eV}$ by varying the diameter from $\sim 3.64$ $\mathrm{nm}$ to $\sim 1.17 \mathrm{~nm}$. This $\Delta E_{g}$ is substantially higher than the near infrared direct band gap of $0.41 \mathrm{eV}$ observed for the bulk PbS. The compressive radial strain on the NWs is found to have a significant effect on their electronic properties. A semiconducting to metallic phase transition occurs at $\zeta=12 \%$ for a representative $\mathrm{NW}$ of $\mathrm{d} \sim 1.98 \mathrm{~nm}$. In addition, we have also observed the strained NW to have an indirect band gap behavior in contrast to the near direct band gap property of the NW. The conduction band of the NW, which has a significant contribution from the excited $3 d$-orbital of $\mathrm{S}$, is found to be more sensitive to the compressive radial strain. The contribution from the $3 d$-orbital of $\mathrm{S}$ at the conduction band minimum develops a bonding characteristic in the part of the $\mathrm{CB}$ wavefuntion, resulting in an energy reduction under CS with the CBM shifting towards and eventually crossing the Fermi energy. Thus, unambiguously, we have identified that the observed phase transition in the recent experiment is due to the CS. The tuning of the electronic structure and hence the bandgap in PbS NWs by varying the diameter of the NWs as well as the external strain on the NWs opens up a new route for their potential applications in nano electronics, optical switches, and solar cells. We present a new orbital-control mechanism to explain the gate field induced switching of current in a semiconducting 
PbSNW junction. An implicit orbital dependent single particle Green's function approach that employ a self-interaction correction scheme is used to calculate the electronic current. A comparative study using different exchange correlation functionals shows a quantitative improvement in the magnitude of current for the self-interaction corrected scheme over the conventional DFT. Both first and higher order Stark effects are included in our model. The consistent increase of calculated current upon increasing negative gate bias as observed in the experiment, and the similar orbital evolution in a $\mathrm{PbSe}$ nanowire junction upon application of gate field reassure the validity of our generalized model, which can also be used to understand switching of current in other lead-chalcogenide NW junctions. Thus, the present work may serve as a guiding point in designing orbital-controlled nanowire-FET for potential applications in new generation electronic circuit. 


\section{Chapter 6}

\section{Spin Transport in Zero-dimensional}

\section{Molecular Junction}

Portion of this chapter is copied from the ACS Nano, vol. 6, page - 3580, year -2012 by Subhasish Mandal and Ranjit Pati. Copyright - Appendix C.

\subsection{Introduction}

A series of successful measurements of electron transport in molecular junctions in recent years has inspired researchers to look for ways to exploit the quantum spin state of the electron with intriguing possibilities of realizing a new paradigm in molecular scale 
electronics $[4,5,6]$. Typically, the spin relaxation time in an organic molecule, which bridges two magnetic electrodes, is longer than the time of flight of the electrons from one electrode to the other electrode. In addition, the chemical flexibility, low cost production as well as the lack of spin-orbit and hyperfine coupling in small organic molecules leading to a longer spin-coherence length make them ideal candidates for exploring coherent spin conserved tunneling. Though spin transport in organic molecular spin valve junction has been studied extensively $[131,132,133,134,135,136]$, several fundamental questions remain elusive. For example, researchers have reported a positive sign [131] for the magnetoresistance in contrast to a negative sign reported by other groups in the same organic spin valve structure $[134,135,136]$.

\subsection{What Determines the Sign Reversal of Tunneling Magnetoresistance?}

In an organic spin valve device structure, where two ferromagnetic electrodes are separated by an insulating or semi-conducting organic layer, the resistance of the circuit depends upon the direction of magnetization at the electrodes. Usually, the device resistance changes from minimal resistance for parallel magnetization (PC) to maximal for anti-parallel magnetization (APC) between the contacts. This gives a positive sign in the magnetoresistance. The negative sign in magnetoresistance arises when the 
device resistance in the case of parallel magnetization is higher than that obtained with the anti-parallel magnetization between the electrodes. Using tris(8-hydroxyquinoline) aluminum (Alq3) organic spacer between two ferromagnetic electrodes, several groups $[134,135,136]$ detected a negative sign in magnetoresistance in contrast to the positive magnetoresistance reported by Barraud et al. [131] in the same spin valve structure. These controversial findings have baffled researchers working in this field [137, 138]. The origin of such anomalous behavior lies in the incomplete understanding of the electronic structure details at the metal-molecule interface as well as the spin polarized electronic structure of the spacer including the effect of bias. Considering the true quantum nature of the problem involving spin state of the electron, first principles theoretical methods are necessary to address this problem. However, there are only a few foremost first principles works on spin transport in single molecular spin-valve junction have been reported $[59,139,140,84,85,142]$. In all of these works the spin-valve actions were demonstrated only at zero bias or at a very small bias range $(\sim \mathrm{mV})$ or using zero-bias spectra - leaving an open and challenging question on the efficiency of the spin-valve when a relatively higher external bias is applied. In addition to the broken symmetry wavefunction due to the opposite alignment of the magnetization at the two electrodes in the anti-parallel configuration, how the external bias further affects the magnetic state is a challenging task to probe within the density functional frame work.

Here, we have constructed a prototypical molecular spin-valve device by sandwiching a planar-organic-molecule 1, 4-Diethynylbenzene between two nickel electrodes to 
investigate the bias dependent spin-valve action [143]. Particularly, we try to answer several questions: What is the reason behind obtaining both positive and negative signs in magnetoresistance for the same spin-valve device structure? How does external bias affect the magnitude as well as the sign of the tunneling magnetoresistance(TMR)? The bias dependent effects (first and higher order Stark effects) are explicitly incorporated in our model. The calculations are carried out by using a first principles spin-polarized, orbital dependent density functional theory (DFT). A parameter-free, single particle Green's function approach $[86,140,84,26,25,10]$ is used to calculate the spin-polarized electronic current.

Our calculations reveal that by changing the interfacial distance $(d)$ from its equilibrium value of $2.06 \AA$ to $2.00 \AA$ ( 3\%change), the sign of TMR changes from a positive to a negative value. In the case of $d=2.00 \AA$, the current in the APC is found to be significantly higher than the PC, resulting in a negative sign in TMR. In contrast, a positive TMR is observed for $d=2.06 \AA$. The large increase in the number of participating eigenstates as well as the change in their orbital character in the APC is found to be responsible for the increase in current. In the APC, the occupied orbitals, which have significant Ni-d character, contribute to the spin-polarized current at $d=2.00 \AA$. On the contrary, at $d=2.06$ $\AA$, unoccupied orbitals, which have only $\mathrm{Ni}-\mathrm{s}$ and $\mathrm{p}$ character, take part in conduction. This clearly suggests that a small change in interfacial distance, which may be generated by thermal fluctuation in the experimental condition, could lead to a different sign in TMR. Apart from this, we quantitatively present the magnetic proximity effect and its 


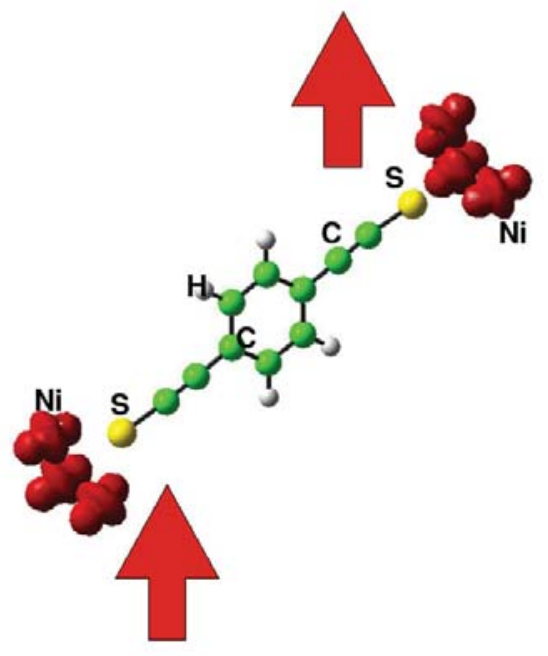

Parallel

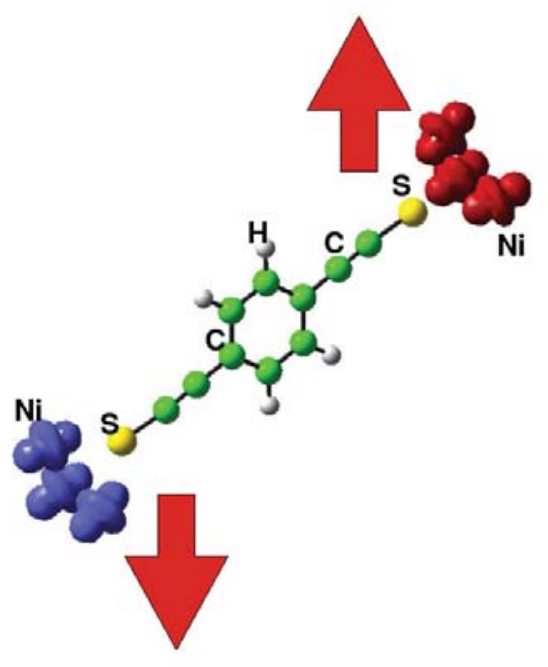

Anti-parallel

(a)

(b)

Figure 6.1: Electron spin density plot for (a) parallel, and (b) anti-parallel alignment of spins at the two electrodes. Red represents positive (up) spin density and blue represents negative (down) spin density. Solid arrow represents the direction of magnetization at the electrodes. Reprinted figure with permission from Subhasish Mandal and Ranjit Pati, ACS Nano 6, 3580 (2012). (c) The American Chemical Society.

bias dependent nature, which can be used to understand the unexpected magnetism often observed [144] in organic materials that are in close proximity with magnetic substrates. At the same time, this work provides a unique pathway to electrical manipulation of quantum spin state, which would help understanding the newly-born "spinterface" science. 


\subsection{Computational Details}

We have used the thiol-substituted 1, 4-Diethynylbenzene (DTB) molecule to build a prototypical spin-valve device. The geometry optimization for the molecule is performed using by DFT, which involves Becke's three-parameter hybrid functional (B3LYP) for exchange-correlation [143]. A real space approach that employs the single determinant many-body wave function is used here. A finite set of Gaussian atomic orbitals [65] is used to construct the wavefunction. The use of real space approach allows us to understand the physical details of transport process through some important quantities, such as spatial distribution of potential, charge and spin densities. We have used all electron 6-311g basis set to represent the atoms in the DTB. The spin-valve junction is constructed by sandwiching the DTB between two ferromagnetic Ni electrodes. For the nickel atoms in the electrode, We have used the LANL2DZ basis set that includes the scalar relativistic effects. The thiolate (-S) anchoring group is used to attach the molecule between the electrodes at the three-fold hollow site of the $\mathrm{Ni}$ (111) surface. It is important to mention that the charge transport properties through this molecule have been investigated very recently by attaching it between Au electrodes [145].

During self-consistent calculation, to ensure an extremely tight convergence, the convergence criterion for energy, maximum, and root mean square electron density is

set at $10^{-10}, 10^{-6}$, and $10^{-8}$ a.u. respectively. We have constructed a strongly-coupled 


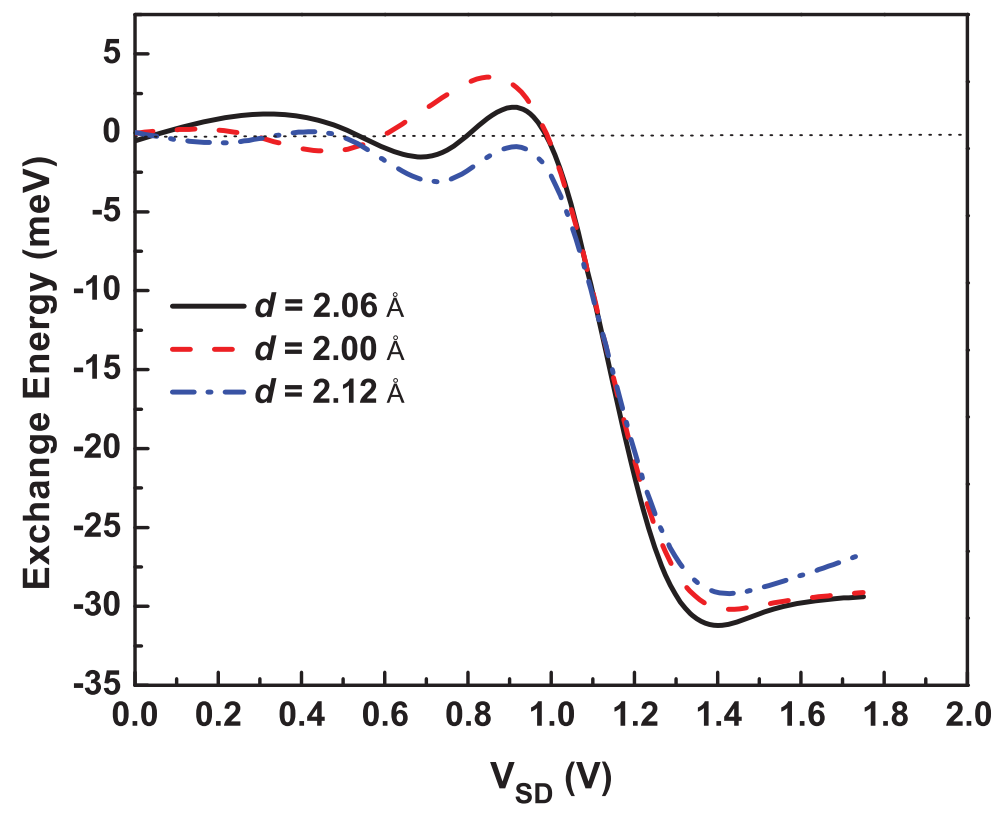

Figure 6.2: Bias dependent exchange energy $\left(E_{P C}-E_{A P C}\right)$ for the spin-valve device with three interfacial distances $(d)$. Reprinted figure with permission from Subhasish Mandal and Ranjit Pati, ACS Nano 6, 3580 (2012). (c) The American Chemical Society.

(chemically-bonded) junction. The interfacial distance $(d)$, which is the distance from the S-atom to the vertex of the triangle formed by the three $\mathrm{Ni}$-atoms of the $\mathrm{Ni}(111)$ surface, is varied for both PC and APC to determine the optimum distance where the repulsive interaction is minimum. For both PC and APC, the optimum distance is found to be $2.06 \AA$. The energy-distance graph yields a parabolic feature around the optimum distance. Since, the metal-molecule interface is the integral part of the device $[132,146]$ that controls the spin transport characteristics, We have considered three different spin-valve structures with $d$ of $2.00,2.06$, and $2.12 \AA$ to investigate the junction dependent TMR. The active scattering 
region at equilibrium is described by the spin-unrestricted Kohn-Sham DFT that requires the solution of an effective single particle Schrodinger-like equation, $H^{\sigma}(0) \psi_{i}^{\sigma}(\vec{r})=$ $\left[-\frac{1}{2} \nabla^{2}+V_{i o n}(\vec{r})+\int d^{3} \vec{r} \frac{\rho(\vec{r})}{\left|(r)-\overrightarrow{r^{\prime}}\right|}+V_{x c}^{\sigma}\right] \times \psi_{i}^{\sigma}(\vec{r})=E_{i}^{\sigma} \psi_{i}^{\sigma}(\vec{r})$

The terms in the bracket represent electron's kinetic energy, ionic potential, coulomb interaction, and the exchange-correlation potential, respectively. The exchange-correlation potential is expressed in terms of the hybrid functional as: $V_{x c}^{\sigma}(\vec{r})=\frac{\delta E_{x c}^{B 3 L Y P}\left[\rho^{\uparrow}, \rho^{\downarrow}\right]}{\delta \rho^{\sigma}}$, where $\sigma=\uparrow, \downarrow$ and $\rho(\vec{r})=\rho^{\uparrow}+\rho^{\downarrow} ; \rho^{\sigma}=\sum_{i} n_{i}^{\sigma}\left|\psi_{i}^{\sigma}(\vec{r})\right|^{2}$. Here $n_{i}^{\sigma}$ is the occupation number of the spin-dependent Kohn-Sham orbital $\psi_{i}^{\sigma}$. It is important to mention that a true dynamical spin-polarized exchange-correlation potential could better represent the transport properties in a molecular junction $[75,129]$. However, considering the complexity of the present problem in dealing with bias-dependent spin-polarized electronic current in a chemically bonded junction, ground-state-based DFT would be a good approximation $[140,79,80,82,83,29]$.

To investigate the spin-transport properties in the DTB molecular spin-valve junction, we have used spin-dependent single-particle Green's function approach which is described in detail in the section 2.5 of Chapter2. 


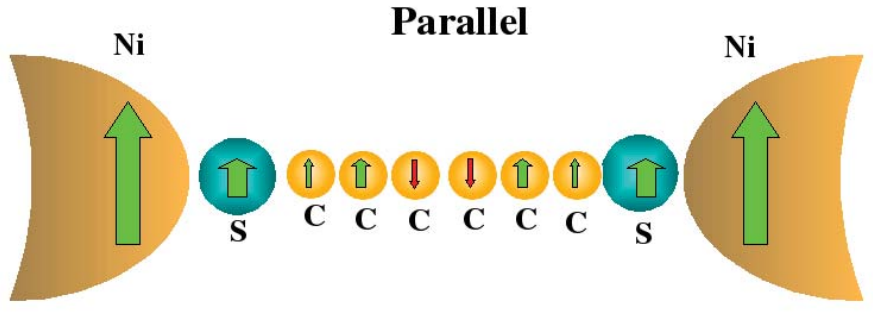

Anti-parallel

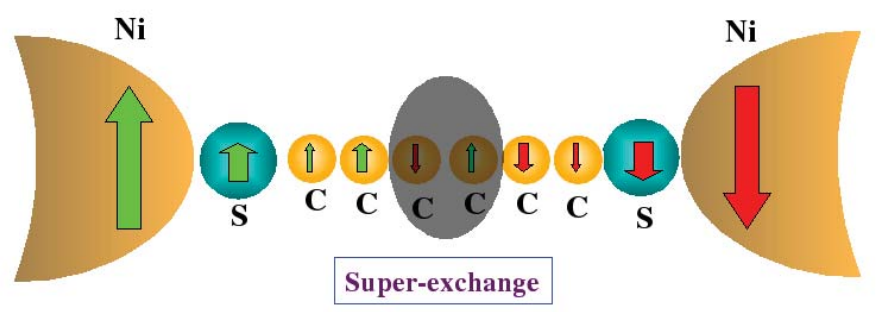

Figure 6.3: Schematic representation of spin-profile along the wire axis for both the parallel and anti-parallel configurations; up(down) arrows refer to the positive (negative) magnetic moment. Reprinted figure with permission from Subhasish Mandal and Ranjit Pati, ACS Nano 6, 3580 (2012). (c) The American Chemical Society.

\subsection{Results and Discussions}

\subsubsection{Spin Density \& Energetics}

In Fig. 6.1, we present calculated spin densities of the device at equilibrium for both PC and APC [143]. For the parallel spin configuration, the total magnetic moment at each electrode is found to be $\sim 3 \mu_{B}$. While for the APC it is found to be $\sim 3 \mu_{B}$ at one electrode and $\sim-3$ $\mu_{B}$ at the opposite electrode. Next, we calculate the exchange energy $\left(E_{e x}\right)$ i.e the energy 
difference between $\mathrm{PC}$ and $\operatorname{APC}\left(E_{P C}-E_{A P C}\right)$ of the extended system. At equilibrium $\left(V_{S D}=0 \mathrm{~V}\right), E_{e x}$ is found to be $-0.0299 \mathrm{meV}$ with APC to be lower in energy than PC. This value is comparable to the energy difference reported earlier in carbon-based molecular spin-valve junctions $[59,85]$. The analysis of spin density distribution in the extended molecule for parallel and anti-parallel configurations (shown in Fig. 6.3) suggests that the stability of the anti-parallel magnetic state is dictated by the super exchange interaction. The bias-dependent $E_{e x}$ for all three interfacial distances are plotted in Fig. 6.2. They all found to exhibit similar behavior in $E_{e x}$ upon applied bias. For a bias range from 0 to $\sim 1 \mathrm{~V}$, the $E_{e x}$ shows a small oscillation. After $1 \mathrm{~V}$, a sudden drop in $E_{e x}(\sim 30 \mathrm{meV})$ is observed. It clearly suggests that the anti-parallel configuration becomes more stable after $V_{S D} \sim 1 \mathrm{~V}$. The enhanced stability of the anti-parallel spin state after $1 \mathrm{~V}$ can be ascribed to the stronger super-exchange interaction caused by the observed equal increase in the magnitude of the magnetic moment at the Nickel site. In contrast, in the parallel magnetic configuration, the increase of bias beyond $1 \mathrm{~V}$ makes the spin distribution asymmetric at the two electrodes (magnitude of the magnetic moments at two electrodes are slightly different) resulting in a decrease in stability. This study thus confirms the manipulation of spin state at the junction by applied bias - a prerequisite for a spin-engineered device. 


\subsubsection{Magnetic Proximity}

When a metallic lead is coupled with a semiconducting molecule, the metallic property of the lead transfers to the semiconducting molecule. Likewise, when a ferromagnetic lead is in close proximity with a non-magnetic material, the non-magnetic material gains some magnetic property due to exchange interaction. This is referred to as magnetic proximity effect [147], which plays an important role in spin injection. In our spin-valve structure, a ferromagnetic lead is in contact with a non-magnetic DTB molecule, allowing the molecular spacer to gain some magnetic property due to proximity effect. How the spin distribution in the non-magnetic molecular spacer, for parallel and anti-parallel spin configuration at the electrodes, is affected by this proximity effect is a challenging question. The other important questions are: How does the applied bias affect the spin distribution in the non-magnetic molecular spacer? How does a change in interfacial distance affect the magnetic character of the spacer? To answer these questions, we looked at the bias dependent acquired magnetic moment of the molecular spacer $\left(M_{\mu}\right)$ in parallel and anti-parallel configurations for three different interfacial distances. In the parallel configuration (Fig. 6.4a), for all three different $d$ s, a similar evolution of $M_{\mu}$ with applied bias is noted. As expected, for a larger $d$, the acquired magnetic moment is found to be smaller. For example, at equilibrium $\left(V_{S D}=0 \mathrm{~V}\right)$, for $d=2.00,2.06$, and $2.12 \AA$, the $M_{\mu}$ is found to be $0.102,0.088$, and $0.074 \mu_{B}$, respectively. It is important to note that, in recent experiments, a magnetic moment of $0.05 \mu_{B}$ per carbon atom was found in $\mathrm{C} / \mathrm{Fe}$ 
multi-layered system [148] and in meteorite graphite [144]. Further inspection of Fig. 6.4a reveals that an increase in applied bias from $0 \mathrm{~V}$ to $\sim 1.2 \mathrm{~V}$ results in a decrease in $M_{\mu}$; a subsequent increase in bias has almost no effect on $M_{\mu}$.

In the case of APC (Fig. 6.4b), as the magnetic moments at the two electrodes are equal and opposite in sign, there is a zero net gain in magnetic moment of the spacer at equilibrium (Fig. 6.3); the atoms in the vicinity of the Ni-electrode having a positive (negative) value of magnetic moment, gain a positive (negative) magnetic moment. For $d=2.06$ and $2.12 \AA$, the $M_{\mu}$ is found to be negative when a finite bias is applied; while for $d=2.00 \AA$, the acquired magnetic moment is found to be positive. For all interfacial distances, the magnitude of the $M_{\mu}$ steadily increases for a bias range of 0 to $\sim 1.2 \mathrm{~V}$. Analogous to the PC, a further increase in applied bias yields almost no effect on the spacer magnetic moment. The origin of such intriguing behavior can be unraveled by understanding the orbital hybridization at the metal-molecule junction. Since the frontier orbitals dictate the electronic and magnetic properties, we have plotted the highest occupied molecular orbitals for the spin up and spin down states in the extended molecule (Fig. 6.5). Several remarks are in order.

First, for PC, the strong orbital hybridization between $\mathrm{Ni}$ and the spacer molecule is found for the spin up state at equilibrium, resulting in a positive magnetic moment in the molecular spacer. As the bias is applied, the symmetry of the wavefunction breaks and the molecular orbital starts to localize in the direction of the electric field; this leads to a decrease in $M_{\mu}$. Increasing the bias beyond $1.2 \mathrm{~V}$ yields almost no change in the strength 
of the hybridization between $\mathrm{Ni}$ and the molecular spacer. This explains the flat nature of the magnetic moment after $V_{S D} \sim 1.2 \mathrm{~V}$. It is important to note that for the $\mathrm{PC}$, the spin down state does not evolve with the bias. Next, turning to the APC, at equilibrium, the contribution from the spin up and spin down states (Fig. 6.5) cancels out, resulting in a net magnetic moment of zero for all three interfacial distances. However, the change in interfacial distance is found to have a significant effect on the bias-dependent orbital evolution. For $d=2.06 \AA$, the spin down states evolve with the increase of bias. In contrast,

for $d=2.00 \AA$, the spin up states evolve. This explains the positive value for $M_{\mu}$ at $d=2.00$ $\AA$ and the negative value for $M_{\mu}$ at $d=2.06 \AA$. A closer inspection reveals that the strength of hybridization between $\mathrm{Ni}$ and the molecule increases with the increase in bias up to $\sim 1.2 \mathrm{~V}$. This leads to an increase in the magnitude of $M_{\mu}$ with bias. Increasing the bias beyond $1.2 \mathrm{~V}$ does not affect the strength of hybridization, and hence $M_{\mu}$ remains almost unchanged.

\subsubsection{Current-voltage}

Next, we turn our discussions to current-voltage (I-V) characteristics of the molecular spin-valve device for three different interfacial distances. The current is calculated within the coherent and spin conserved tunneling limit [143]. The results are summarized in Fig. 6.6. Total current for both PC $\left(I_{P C}\right)$ and APC $\left(I_{A P C}\right)$ is obtained by adding the currents for the spin-up and spin-down states. The contribution to the current from the spin up and spin 
down states is almost identical in the case of APC; while for the PC, the spin up contribution is higher than that for the spin down states for all three different $d$ s. A non-linear feature in $\mathrm{I}-\mathrm{V}$ is noticeable for all three different $d \mathrm{~s}$.

From Fig. 6.6, a similar trend in I-V is noted for $d=2.06$ and $2.12 \AA$; the current in the PC is found to be higher than the APC. For $d=2.00 \AA$, the current in PC is found to be higher than the APC only for a small bias range of 0 to $\sim 0.25 \mathrm{~V}$; after $0.25 \mathrm{~V}$, with increasing bias, the $I_{A P C}$ is found to be significantly higher than the $I_{P C}$. A closer examination reveals the $I_{A P C}$ depends sensitively on the interfacial distance. By changing the $d$ from 2.06 to $2.00 \AA$, the current in the APC is found to increase by $\sim 4$ times at $\sim 1 \mathrm{~V}$. In contrast, for the PC, the change in $d$ from 2.06 to $2.00 \AA$ yields a decrease in current by 0.86 times.

\subsubsection{Tunneling Magnetoresistance}

To quantify the spin-valve action in detail, we calculate the TMR as: $T M R=\frac{I_{P C}-I_{A P C}}{\left(I_{P C}+I_{A P C}\right) / 2} \times$ 100\%. Fig. 6.7 shows TMR as a function of bias voltage for three different interfacial distances. As noted from Fig. 6.6, spin-valve structures with $d=2.06$ and $2.12 \AA$ yield a similar characteristic in TMR; TMR is positive and the magnitude of TMR is found to decrease with an increase in bias up to $1.25 \mathrm{~V}$ and then remains flat. For $d=2.00 \AA$, TMR is found to be positive only for a bias range of 0 to $\sim 0.25 \mathrm{~V}$; a subsequent increase in bias yields a negative TMR which remains flat after $V_{S D} \sim 1.25 \mathrm{~V}$. For example, at $V_{S D}=1.00 \mathrm{~V}$, 
the TMR for $d=2.00 \AA$ is $-113.6 \%$, while for $d=2.06 \AA$ the TMR is $+22.39 \%$. The similar bias-dependent behavior is also noted in Fig. 6.4 where the acquired magnetic moment of the molecule remains almost flat after $V_{S D} \sim 1.2 \mathrm{~V}$.

\subsubsection{Transmission}

To gain deeper insight into the origin of sign reversal in TMR, we calculated spin-polarized bias dependent transmission for three different interfacial distances. Since both $d=2.06$ and $2.12 \AA$ show similar trends in TMR, we present spin-polarized transmission for the contrasting cases i.e for $d=2.00$ and $2.06 \AA$ in Fig. 6.8. For brevity, we have presented our results at $V_{S D} \sim 1 \mathrm{~V}$. The dotted lines represent the chemical potential window (CPW). In

the case of $d=2.06 \AA$, the spin up transmission for PC is higher than for APC. This gives a higher net transmission (sum of spin up and spin down transmission) in PC than in APC. For example, with $d=2.06 \AA$, at injection energy $(E)=-0.45 \mathrm{eV}$, the total transmission for $\mathrm{PC}$ is 0.185 , while the transmission for APC is 0.150 . This explains the observed higher current for PC than APC at $d=2.06 \AA$ (Fig. 6.6(a)) resulting in a positive TMR (Fig. 6.7). Next, we discuss the transmission for $d=2.00 \AA$. We notice that the spin up and spin down transmission for the PC is significantly smaller than that for APC. The total transmission at $E=-0.45 \mathrm{eV}$ for $\mathrm{PC}$ is 0.159 and for APC is 0.704 . This $\sim 4$ times increase in transmission for APC is attributed to the $\sim 4$ times increase in current at $\sim 1 \mathrm{~V}$ for APC (Fig. 6.6(c)). 
Now, a natural question arises: What causes the transmission to behave differently for different $d$ s? To understand this, we looked at the eigen-channels that contribute to the transmission within the CPW. For APC, the number of eigen-channels within the chemical potential window increases significantly as we change the $d$ from 2.06 to $2.00 \AA$. For $d=2.06 \AA$, mainly frontier unoccupied levels having $\mathrm{Ni}$ (s,p) characters contribute to the conduction; while for $d=2.00 \AA$, all the participating orbitals for APC are found to be occupied with a significant Ni-d character. This suggests that a small change in intefacial distance can have a significant effect on the electronic structure of the device, which could lead to the observed sign reversal in TMR.

\subsubsection{Molecule-lead Coupling}

Since the conduction in molecular spin-valve junction not only depends on the electronic structure of the molecular spacer but also on the electronic structure at the metal-molecule interface, we looked at the role of molecule-lead coupling on electronic current to discern the junction effect [143]. We recalculated the $I_{P C}$ at $1.0 \mathrm{~V}$ for $d=2.00 \AA$ (where the TMR is found to be negative) using $V_{L M}^{\sigma}$ and $V_{M R}^{\sigma}$ (eq. 2.43) extracted from APC at the same bias. The spin up current in the PC changes from 2.896 to $3.432 \mu \mathrm{A}$, while the spin down current changes from 0.923 to $7.972 \mu \mathrm{A}$; this leads to an increase in total current of $\sim 3$ times. Similarly, we recalculated the current for the PC for $d=2.06 \AA$ (where the TMR is found to be positive) using $V_{L M}^{\sigma}$ and $V_{M R}^{\sigma}$ extracted from the APC at the same bias, for 
the spin up and spin down state, respectively. the total current is found to decrease. This clearly reflects that a small change in interfacial distance can have a significant effect on the molecule-lead coupling and hence on the escape rate of the electrons.

\subsection{Conclusions}

We have studied spin-polarized transport properties in a prototypical spin-valve junction, which is constructed by sandwiching a 1, 4-Diethynylbenzene planar, organic molecule between two nickel electrodes. A spin-unrestricted, orbital dependent density functional theory in conjunction with a single particle Green's function approach is used to calculate the spin-polarized current. Bias effects are explicitly included in our calculations. Our calculation shows a small change of $\sim 3 \%$ in metal-molecule interfacial distance can alter the sign of tunneling magnetoresistance in a molecular spin-valve device. The current in the APC is found to be strongly affected by the change in the interfacial distance. The higher current in the APC for certain $d$ is attributed to the increase in the number of eigen-channels with significant Ni-d characters. Thus, this work not only provides an explanation for the sign reversal of TMR in a molecular tunnel device at the electronics structure level but it also opens up a new pathway for orbital manipulation in molecular spintronics. 


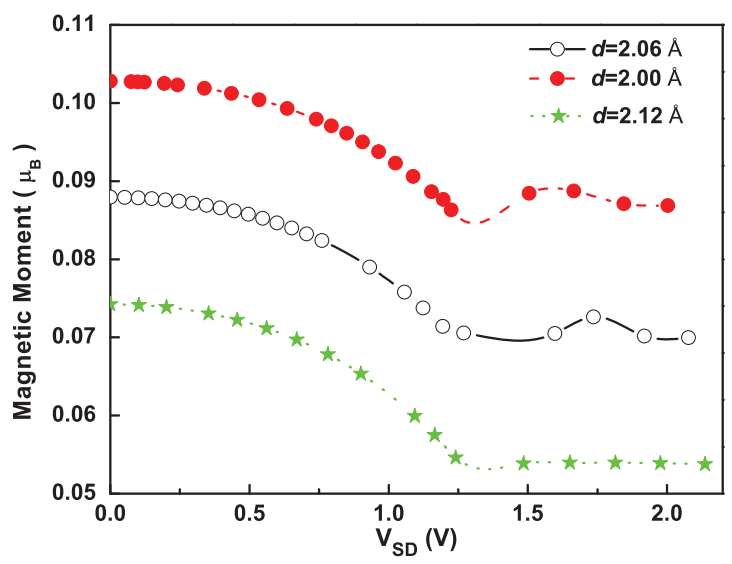

(a)

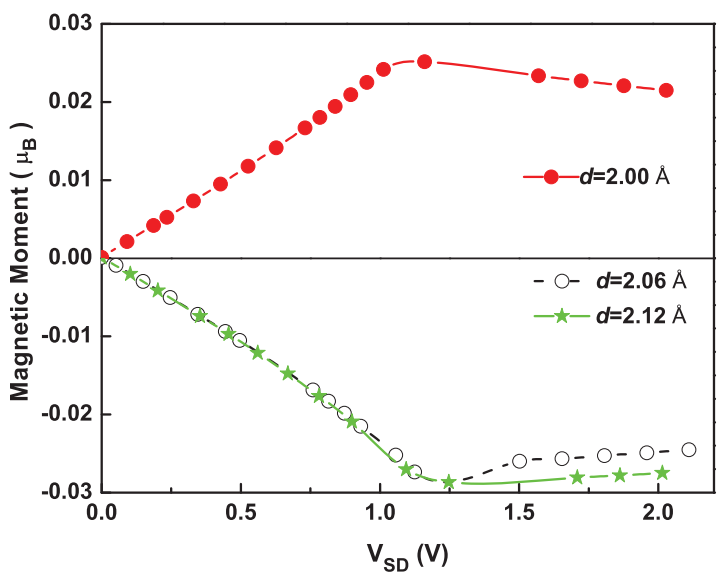

(b)

Figure 6.4: Bias dependent magnetic moment of the molecular spacer for (a) parallel, and (b) anti-parallel alignment of spins at two electrodes. $d$ refers to the interfacial distance. Reprinted figure with permission from Subhasish Mandal and Ranjit Pati, ACS Nano 6, 3580 (2012). (c) The American Chemical Society. 


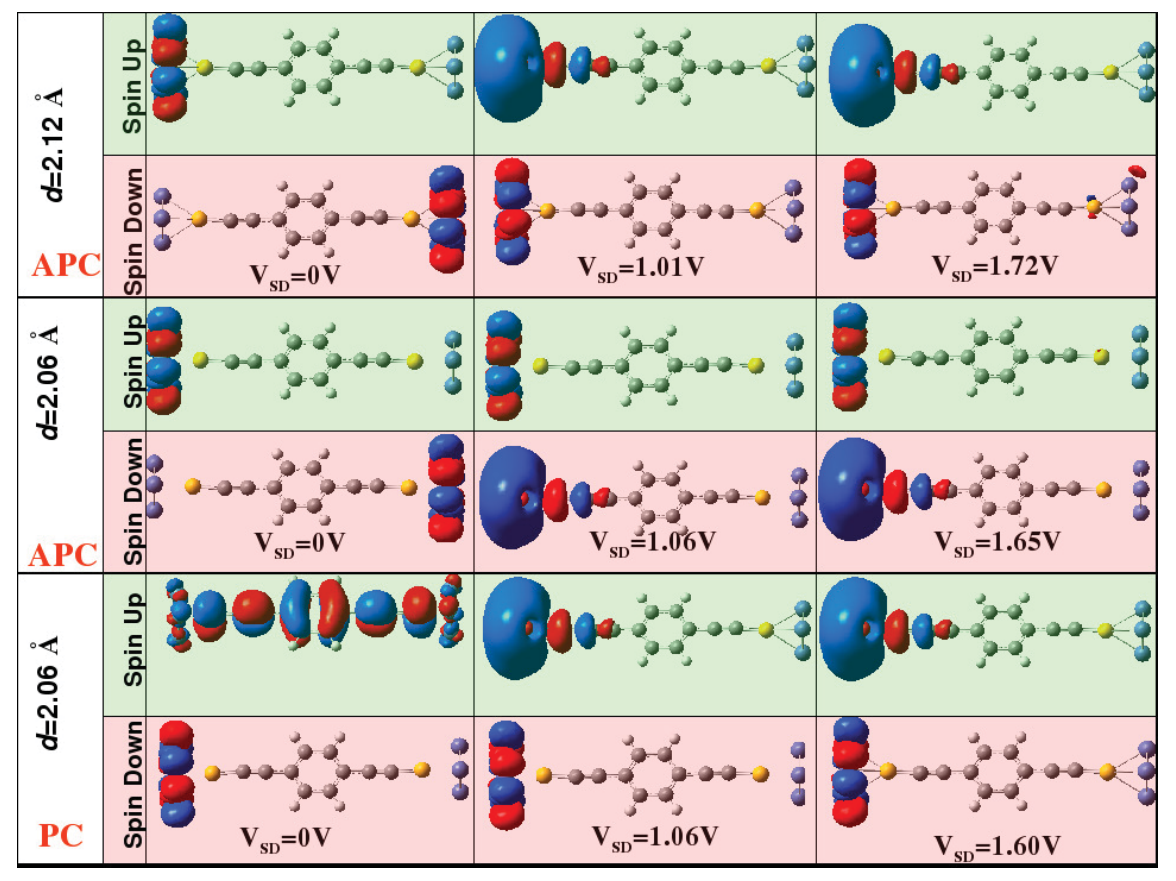

Figure 6.5: Bias dependent molecular orbital for HOMO; panel in green shows molecular orbital for spin up states while panel in red shows for spin down states. Reprinted figure with permission from Subhasish Mandal and Ranjit Pati, ACS Nano 6, 3580 (2012). (c) The American Chemical Society. 


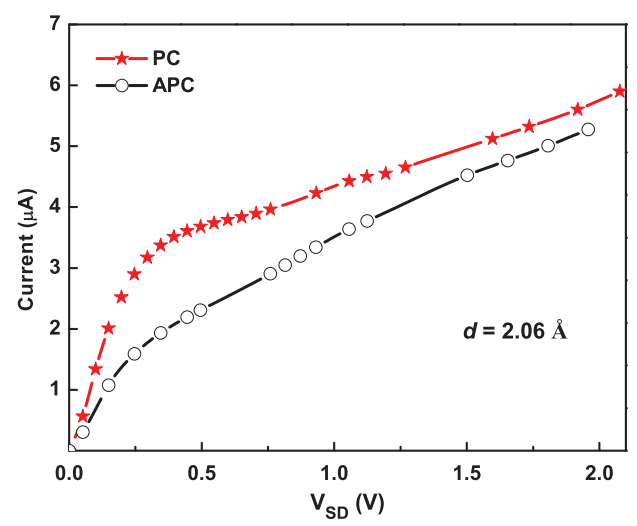

(a)

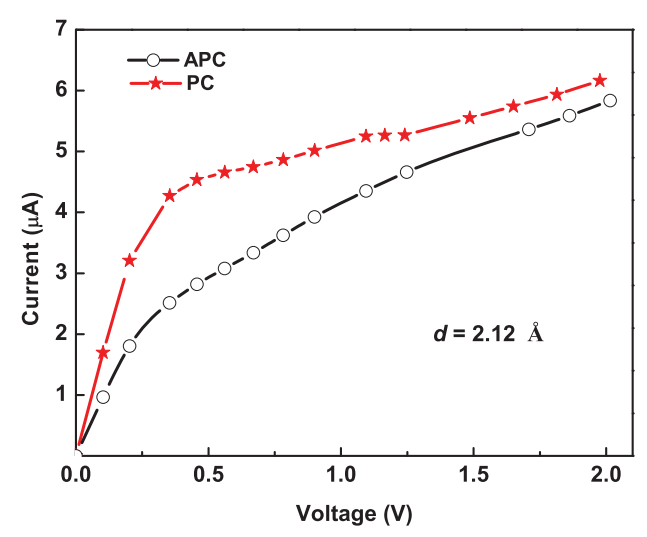

(b)

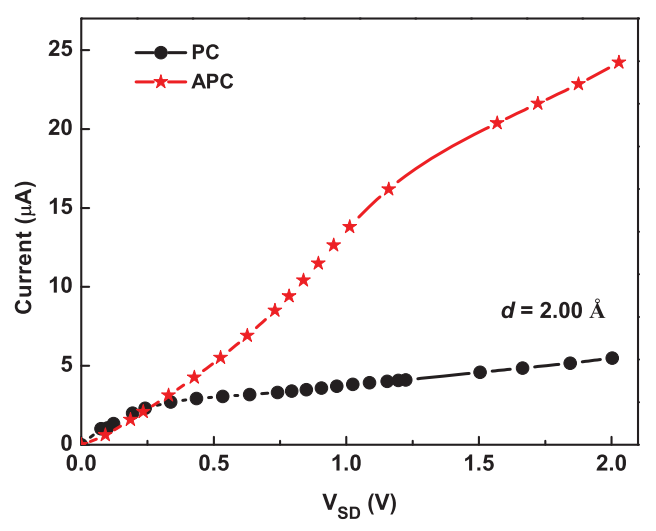

(c)

Figure 6.6: Current-voltage characteristics for the parallel and anti-parallel configurations with $d=$ (a) 2.06, (b) 2.12, and (c) $2.00 \AA$ A. Reprinted figure with permission from Subhasish Mandal and Ranjit Pati, ACS Nano 6, 3580 (2012). (C) The American Chemical Society. 


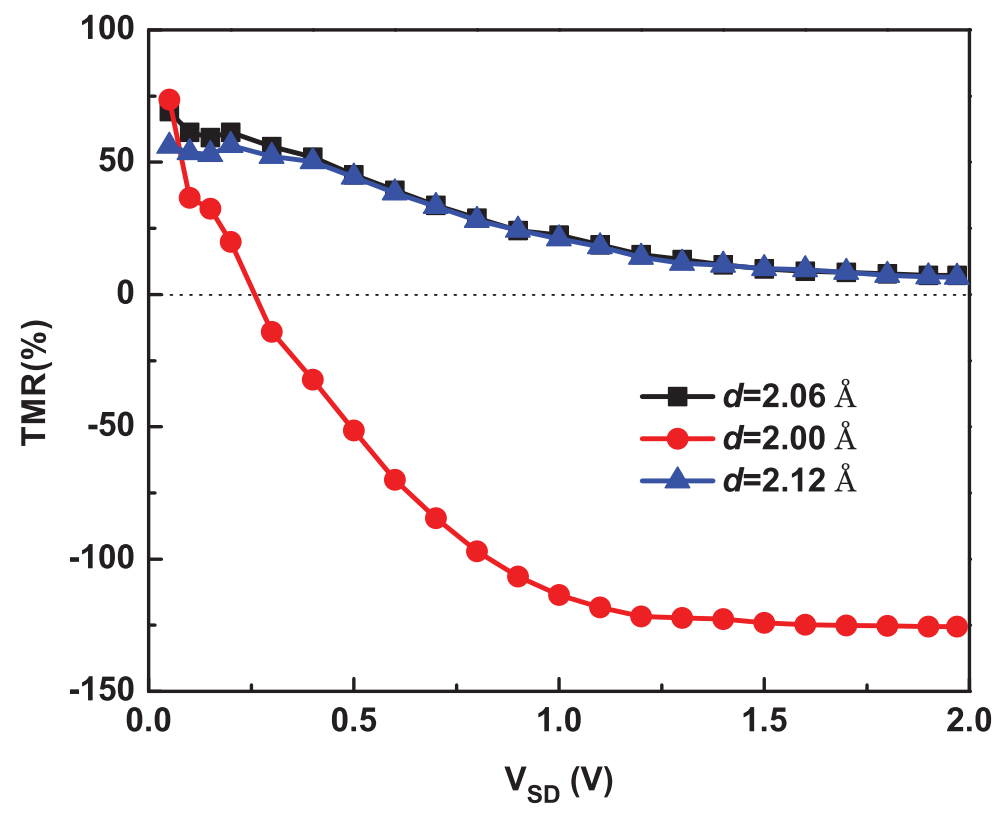

Figure 6.7: Bias dependent tunnel magnetoresistance for three interfacial distances $(d)$. Reprinted figure with permission from Subhasish Mandal and Ranjit Pati, ACS Nano 6, 3580 (2012). (c) The American Chemical Society. 


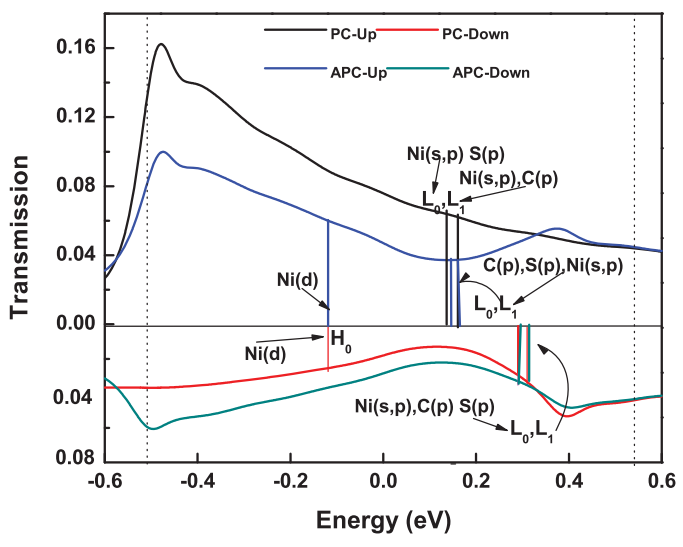

(a)

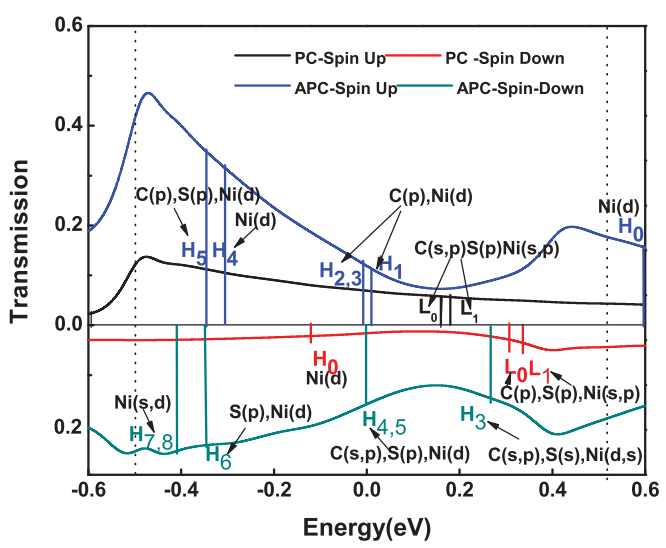

(b)

Figure 6.8: Bias dependent transmission function for $d=$ (a) 2.06 and (b) $2.00 \AA$. The Fermi level lies at $E=0$. The dotted lines show the chemical potential window. $H_{0}, H_{1}, H_{2}, H_{3}, H_{4}, H_{5}, H_{6}, H_{7}, H_{8}$ represent HOMO, HOMO-1, HOMO-2, HOMO-3, HOMO-4 HOMO-5, HOMO-6, HOMO-7, and HOMO-8, respectively. $L_{0}$ and $L_{1}$ represent LUMO and LUMO+1, respectively. Reprinted figure with permission from Subhasish Mandal and Ranjit Pati, ACS Nano 6, 3580 (2012). (c) The American Chemical Society. 


\section{Chapter 7}

\section{Summary}

In this chapter, I summarize the finding of the projects that compiled this dissertation. We have used a DFT-based single particle Green's function approach, which involves B3LYP functional for exchange-correlation. The use of explicit orbital dependent B3LYP functional allows us to include a part of the exact Hatree-Fock exchange and thus corrects partly the self-interaction error. we have shown this partly self-interaction corrected scheme can yield a lower current value that is in better agreement with the experimentally measured current, when compared with the calculated current obtained using other functionals (SVWN, PW91PW91, PW91LYP). First, we investigated transport properties in single molecular junctions. We studied a new codoping approach in a single molecular carborane junction, where a cation and an anion are simultaneously

doped to find the role of a single atom in the device. The main purpose was to build a 
molecular junction where a single atom can dictate the flow of electron in a circuit. When compared to the undoped system, at $\sim 2 \mathrm{~V}$, we found an order of magnitude increase in the current value in the $\mathrm{Na} / \mathrm{B}$-codoped system. Comparing to the current value in the Li/B-codoped system, a two fold increase in the current is observed at $\sim 2 \mathrm{~V}$ in the $\mathrm{Na} / \mathrm{B}$-codoped system; this suggests that the single alkali atom dictates the electron flow in codoped junction. Further analysis reveals that alkali atoms donate charge to the $C_{1} B_{11} H_{10} S_{2}$ host; the amount of charge transfer varies with the applied bias. This research thus opens up the door toward an ultimate limit of the miniaturization, where a single atom controls the device characteristics. Subsequently, we studied the electronic structure properties of this nanowire and investigated the quantum confinement effect by calculating the bandgap of $\mathrm{PbS}$ nanowires with different diameters. We found the bandgap decreases as we increase the diameter -confirming the quantum confinement effect in $\mathrm{PbS}$ nanowire. Subsequently, we found the explanation of an observed phase transition of this nanowire. By calculating the bandgap of the nanowire under uniform radial strain, we found the compressive radial strain on the nanowire is responsible for the metallic to semiconducting phase transition. Next, we used this nanowire to build a field effect transistor. The goal was to model a nanowire field effect transistor and to understand the gate-field induced switching phenomena. A new orbital-control mechanism to explain the gate field induced switching of current in a semiconducting PbSNW junction has been proposed in this dissertation. This mechanism can be used to understand the gate field induced switching not only in $\mathrm{PbS}$ nanowire but also in other lead chalcogenides 
nanowire. Next, the focus was turned to the spin transport in single molecular junction. We have studied spin-polarized transport properties in a prototypical spin-valve junction, which is constructed by sandwiching a 1, 4-Diethynylbenzene planar, organic molecule between two nickel electrodes. A spin-unrestricted, orbital dependent density functional theory in conjunction with a single particle Green's function approach is used to calculate the spin-polarized current. Bias effects are explicitly included in our calculations. Our calculation shows a small change of $\sim 3 \%$ in metal-molecule interfacial distance can alter the sign of tunneling magnetoresistance in a molecular spin-valve device. The current in the APC is found to be strongly affected by the change in the interfacial distance. The higher current in the APC for certain $d$ is attributed to the increase in the number of eigen-channels with significant Ni-d characters. Thus, this work not only provides an explanation for the sign reversal of TMR in a molecular tunnel device at the electronics structure level but it also opens up a new pathway for orbital manipulation in molecular spintronics. 



\section{References}

[1] G. E. Moore, Electronics, 38, 8 (1965).

[2] A. Aviram, and M. A. Ratner, Chem. Phys. Lett. 29, 277 (1974).

[3] H. Song, Y. Kim, Y. H. Jang, H. Jeong, M. A. Reed, T. Lee, Nature 462, 1039 (2009).

[4] M. A. Reed, C. Zhou, C. J. Muller, T. P. Burgin, J. M. Tour, Science 278, 252 (1997).

[5] B. Xu, N. J. Tao, Science 301, 1221 (2003).

[6] J. Zhou, F. Chen, B. Xu, J. Am. Chem. Soc. 131, 10439 (2009).

[7] N. J. Tao, Nature Nanotechnology 1,173 (2006).

[8] J. Chen, M. A. Reed, A. M. Rawlett, and J. M. Tour, Science 286, 1550 (1999).

[9] S. Sahoo, T. Kontos, J. Furer, C. Hoffmann, M. Graber, A. Cottet, and C. Schonenberger, Nat. Phys. bf 1, 99 (2005).

[10] R. Pati, M. McClain, A. Bandyopadhyay. Phys. Rev. Lett. 100, 246801 (2008). 
[11] K. Burke, R. Magyar, The ABC of DFT "http://dft.uci.edu/sites/default/files/g1.pdf"

[12] M. C. Payne, M. P. Teter, D. C. Allan, T. A. Arias, J. D. Joannopoulos, Rev. Mod. Phys. 64, 1045 (1992).

[13] R. E. Cohen, Nature, 358, 136 (1992).

[14] R. Pati, Theory of Electronic Structures and Nuclear Quadrupole Interactions in Molecular Solids and Semiconductors Surfaces. Doctoral Thesis, State University of New York at Albany, 23, 1998.

[15] P. Panigrahi, Controlling Electronic and Magnetic Properties of Ultra Narrow Multilayered Nanowires. Doctoral Thesis, Michigan Technological University. 13-39, 2009.

[16] M. Born, J. R. Oppenheimer, Ann. Physik. 84, 457 (1927).

[17] R. G. Parr and W. Yang, Density-Functional Theory of Atoms and Molecules ( Oxford University Press, Oxford, England, 1989).

[18] H. Toffoli, Online lecture notes "http://www.physics.metu.edu.tr/ hande/teaching/741.html".

[19] A. Szabo, N. S. Ostlund, Modern Quantum Chemistry ( Dover Publishingm Mineola, New York, 1996).

[20] P. Hohenberg, W. Kohn, Phys. Rev. 136, B864 (1964).

[21] W. Kohn, L. J. Sham, Phys. Rev. 140, A1133 (1965). 
[22] W. Kohn, Rev. Mod. Phys. 71, 1253 (1992).

[23] J. P. Perdew, K. Burke, M. Ernzerhof, Phys. Rev. Lett. 77, 3865 (1996).

[24] A. D. Becke, J. Chem. Phys. 98, 5648 (1993).

[25] M. Di Ventra, Electrical Transport in Nanoscale Systems, (Cambridge, New York, 2008).

[26] S. Dutta, Electron Transpot in Mesoscopic System ( Cambridge University Press, Cambridge, England, 1997).

[27] S. Mandal, and R. Pati, Phys. Rev. B 83, 195420 (2011).

[28] P. Pal, R. Pati, Phys. Rev. B 82, 045424 (2010).

[29] S. Mandal, R. Pati, Phys. Rev. B 84, 115306 (2011).

[30] B. G. Johnson, P. M. W. Gill, J. A. Pople, D. J. Fox, Chem. Phys. Lett. 206, 239 (1993).

[31] J. M. Seminario, J. Phys. B: At. Mol. Opt. Phys. 40, F275-F276 (2007).

[32] I. P. Batra, Solid State Commun. 124, 463 (2002).

[33] R. Landauer, IBM J. Res. Dev. 1, 223 (1957).

[34] R. Landauer, J. Phys. Cond. Matt. 1, 8099 (1989).

[35] P. P. Pal, Quantum Transport in a Single Molecular Junction, Doctoral Thesis, Michigan Technological University. 26-33, 2011. 
[36] G. D. Mahan, Many-Particle Physics ( Kluwer Academic/Plenum Publishers, New York, 2000).

[37] M. Galperin, M. A. Ratner, and A. Nitzan, Nano Lett. bf 5, 126, 2005.

[38] S. C. Erwin, L. Zu, M. I. Haftel, A. L. Efros, T. A. Kennedy, D. J. Norris Nature 436, 91 (2005).

[39] D. J. Norris, A. L. Efros, S.C. Erwin, Science 319, 1176 (2008).

[40] M. -H. Du, S. C. Erwin, A. L. Efros, Nano Lett. 8, 2878 (2008).

[41] A. P. Alivisatos, Science 271, 933 (1996).

[42] O. Seitz, A. Vilan, H. Cohen, C. Chan, J. Hwang, A. Kahn, C. Cahen, J. Am. Chem. Soc. 129, 7494 (2007).

[43] X. Zhong, R. Pandey, A. R. Rocha, S. P. Karna, Phys. Chem. Lett. 1, 1584 (2010).

[44] M. J. Rosseinsky, A. P. Ramirez, S. H. Glarum, D. W. Murphy, R. C. Haddon, A. F. Hebard, T. T. M. Palstra, A. R. Kortan, S. M. Zahurak, A. V. Makhija, Phys. Rev. Lett. 66, 2830 (1991).

[45] A. F. Hebard, M. J. Rosseinsky, R. C. Haddon, D. W. Murphy, S. H. Glarum,T. T. M. Palstra, A. P. Ramirez, A. R. Kortan, Nature 350 , 600 (1991).

[46] K. Holczer, O. Klein, S. -M. Huang, R. B. Kaner, K. -J Fu, R. L. Whetten, F. Diederich, Science 252, 1154, (1991). 
[47] S. P. Kelty, C. -C. Chen, C. M. Lieber, Nature 352, 223 (1991).

[48] R. Yamachika, M. Grobis, W. M. F. Crommie, Science 304, 281 (2004).

[49] S. R. Schofield, N. J. Curson, M. Y. Simmons, F. J. Rue $\beta$, T. Hallam, L. Oberbeck, R. G. Clark, Phys. Rev. Lett. 91, 136104 (2003).

[50] P. F. H. Schwab, M. D. Levin, J. Michl, Chem. Rev.99, 1863 (1999).

[51] J. R. Heath, M. A. Ratner, Phys. Today 56, 43 (2003).

[52] L. I. Zakharkin, A. L. Kovredov, Zh. Obshch. Khim. 44, 1840 (1974).

[53] L. I. Zakharkin, A. L. Kovredov, Izv. Akad. Nauk. SSR, Ser. Khim. 1428 (1973).

[54] X. G. Yang, W. Jiang, C. B. Knobler, M. F Hawthorne, J. Am. Chem. Soc. 114, 9719 (1992).

[55] J. Muller, K. Base, T. F. Magnera, J. Michl, J. Am. Chem. Soc. 114, 9721 (1992).

[56] A. Franken, C. A. Kilner, J. D. Kennedy, Chem. Commun. 3, 328 (2004).

[57] R. N. Grimes, J. Chem. Educ. 81, 658 (2004).

[58] S. Morandi, S. Ristori, D. Berti, L. Panza, A. Beccioloni, G. Martini, Biochim. Biophys. Acta-Biomembranes. 1664, 53 (2004).

[59] R. Pati, A. C. Pineda, R. Pandey, S. P. Karna, Chem. Phys. Lett. 406, 483 (2005).

[60] W. D. Jemmis, M. M. Balakrishnarajan, J. Am. Chem. Soc. 122, 7392 (2000). 
[61] J. M. Oliva, L. Serrano-Andres, D. J. Klein, P. v. R. Scheleyer, J. Michl, Int. J. Photoenergy. 2009, 292393 (2009).

[62] L. Serrano-Andres, J. M. Oliva, Chem. Phys. Lett. 432, 235 (2006).

[63] W. Zhu, X. Qiu, V. Iancu, X. -Q. Chen, H. Pan, W. Wang, N. M. Dimitrijevic, T. Rajh, H. M. Meyer, M. P. Paranthaman, G. M. Stocks, H. H. Weitering, B. Gu, G. Eres, Z. Zhang, Phys. Rev. Lett. 103, 226401, (2009).

[64] R. G. Parr, W. Yang, Density Functional Theory of Atoms and Molecules ( Oxford, Science, 1994).

[65] GAUSSIAN 03, Gaussian Inc., Pittsburg, PA, 2003.

[66] J. Zhou, F. Hagelberg, Phys. Rev. Lett. 97, 045505 (2006).

[67] F. Evers, K. Burke, Nano and Molecular Electronics Handbook (CRC Press, 2007).

[68] P. Delaney and J. C. Greer, Phys. Rev. Lett. 93, 036805 (2004).

[69] G. Fagas, P. Delaney, J. C. Greer, Phys. Rev. B 73, 241314 (2006).

[70] I. Lindgren, J. Morrison, Atomic Many Body Theory edited by Lambropoulos and H Walther,( Berlin, Springer, 1985).

[71] K. S. Thygesen, A. Rubio, Phys. Rev. B 77, 11533 (2008)

[72] N. Sai, M. Zwolak, G. Vignale, M. D. Ventra, Phys. Rev. Lett. 94, 186810 (2005).

[73] C-L. Cheng, J. S. Evans, T. V. Voorish, Phys. Rev. B 74, 15112 (2006). 
[74] F. Evers, F. Weigend, M. Koentopp, Phys. Rev. B 69, 235411 (2004).

[75] E. Runge and E. K. U. gross, Phys. Rev. Lett. 52, 997 (1984).

[76] K. Burke, R. Car, and R. Gebauer, Phys. Rev. Lett. 94, 146803 (2005).

[77] X. Gonze and M. Scheffler, Phys. Rev. Lett. 82, 4416 (1999).

[78] J. Taylor, H. Guo, J. Wang, Phys. Rev. B 63, 245407 (2001).

[79] M. Brandbyge, J. L. Mozos, P. Ordejon, J. Taylor, K. Stokbro, Phys. Rev. B 65, $165401(2002)$.

[80] Y. Xue, S. Datta, M. A. Ratner, J. Chem. Phys. 115, 4292 (2001).

[81] M. Di Ventra, S. T. Pantelides, and N. D. Lang, Phys. Rev. Lett. 84, 979 (2000).

[82] W. Su, J. Jiang, W. Lu, and Y. Luo, Nano. Lett. 6, 2091 (2006).

[83] G. C. Solomon, C. Herrmann, T. Hansen, V. Mujica, M. A. Ratner, Nature Chem. 2, 223 (2010).

[84] A. R. Rocha, V. M. Garcia-suarez, S. W. Bailey, C. J. Lambert, J. Ferrer, S. Sanvito, Nature Mat. 4, 335 (2005).

[85] R. Pati, L. Senapati, P. M. Ajayan, S. K. Nayak, Phys. Rev. B 68, 100407 (2003).

[86] A. Nitzan, M. A. Ratner, Science 300, 1384 (2003).

[87] Vienna ab initio Simulation Package, Technische Universität Wien, 1999; G. Kresse, J. Furthmüller, Phys. Rev. B 54, 11169 (1996). 
[88] Y. Meir, N. S. Wingreen, Phys. Rev. Lett. 68, 2512 (1992).

[89] R. K. Bohn, M. D. Bohn, Inorg. Chem. 10, 350 (1971).

[90] P. v. R. Schleyer, K. Najafian, Inorg. Chem. 37, 3454 (1998).

[91] T. P. Fehlner, M. Wu, B. J. Meneghelli, R. W. Rudolph, Inorg. Chem. 19, 49 (1980).

[92] J. Xiang, W. Lu, Y. Hu, Y. Wu, H. Yan, and C. M. Lieber, Nature 441, 489 (2006).

[93] H. Yan, H. S. Choe, S. Nam, Y. Hu, S. Das, J. F. Klemic, J. C. Ellenbogen, and C. M. Lieber, Nature 470, 240 (2011).

[94] Yu Huang, Xiangfeng Duan, Yi Cui, Lincoln J. Lauhon, Kyoung-Ha Kim, Charles M. Lieber, Science 294, 1313 (2001).

[95] Yi Cui, Zhaohui Zhong, Deli Wang, Wayne U. Wang, and Charles M. Lieber, Nano Lett. 3, 149 (2003).

[96] Nicholas A. Melosh, Akram Boukai, Frederic Diana, Brian Gerardot, Antonio Badolato, Pierre M. Petroff, James R. Heath, Science 300, 112 (2003).

[97] Fernando Patolsky, Gengfeng Zheng, Oliver Hayden, Melike Lakadamyali, Xiaowei Zhuang and Charles M. Lieber, Proceedings of the National Academy of Sciences, U.S.A, 10114017 (2004).

[98] Yi Cui, Qingqiao Wei, Hongkun Park, Charles M. Lieber, Science 293, 1289 (2001). 
[99] Eric Stern, James F. Klemic, David A. Routenberg, Pauline N. Wyrembak, Daniel B. Turner-Evans, Andrew D. Hamilton, David A. LaVan, Tarek M. Fahmy, Mark A. Reed, Nature, 445, 519 (2007).

[100] V. L. Colvin, M. C. Schlamp, and A. P. Alivisatos, Nature 370, 354 (1994).

[101] Wanli Ma, Joseph M. Luther, Haimei Zheng, Yue Wu, A. Paul Alivisatos, Nano Lett. published online on 12 March 2009.

[102] Matt Law, Matthew C. Beard, Sukgeun Choi, Joseph M. Luther, Mark C. Hanna, and Arthur J. Nozik, Nano Lett. 8, 3904 (2008).

[103] Robert Plass, Serge Pelet, Jessica Krueger, Michael Gratzel, Udo Bach, J.Phys. Chem B 106, 7578 (2002).

[104] I. Patla, S. Acharya, L. Zeiri, J. Israelachvili, S. Efrima, Y. Golan, Nano Lett. 71459 (2007).

[105] P. K. Mukherjee, K. Chatterjee, and D. Chakravorty. Phy. Rev B 73, 035414 (2006).

[106] F. Gao, Q. Lu, X. Liu, Y. Yan, and D. Zhao, Nano Lett. 1, 743 (2001).

[107] H. Yu, J. Li, R. A. Loomis, L-W Wang, W. E. Buhro, Nature Materials, 2, 517 (2003).

[108] Q. Wang, Q. Sun, P. Jena, Y. Kawazeo, Nano Lett. 5, 1587 (2005).

[109] Vincent Meunier et. al., Nano Lett. published online on 18 March, 2009. 
[110] W. Liang, A. I. Hochbuam, M. Fardy, O. Rabin, M. Zhang, P. Yang, Nano Lett. published online on 24 March 2009.

[111] I. U. Arachchige, M. G. kanatzidis, Nano Lett. published online on 24 March 2009.

[112] Z. Zanolli, M-E Pistol, L E Frogberg, L Samuelson, J. Phys. Condens. Matter 19, 295219 (2007).

[113] G. Nimtz and B. Schlicht, Narrow-Gap Semiconductors Springer-Verlag, New York, (1985).

[114] Matthew J. Bierman, Y. K. Albert Lau, and Song Jin, Nano Lett. 7, 2907 (2007).

[115] S. Mandal, and R. Pati, Chem. Phys. Lett. 479, 312 (2009).

[116] Xinyuan Zhao, C. M. Wie, L. Yang, M. Y. Chou, Phys. Rev. Lett. 92, 236805 (2004).

[117] X.-H. Peng, S. Ganti, A. Alizadeh, P. Sharma, S. K. Kumar, S. K. Nayak Phys. Rev. B 2006, 74, 035339

[118] A. K. Singh, V. Kumar, R. Note, Y. Kawazoe, Nano Lett. 6, 920 (2006).

[119] K-H. Hong, J. Kim, S-H. Lee, J. K. Shin, Nano Lett. 8 (2008) 1335.

[120] M. Nolan, S. O’Callaghan, G. Fagas, J. C. Greer, Nano Lett. 7, 34 (2007).

[121] Z. Wu, J. B. Neaton, J. C. Grossman, Phys. Rev. Lett. 100 (2008) 246804.

[122] D. V. Talapin and C. B. Murray, Science 310, 86 (2005). 
[123] K-S. Cho, D. V. Talapin, W. Gaschler, and C. B. Murray, J. Am. Chem. Soc. 127, $7140(2005)$.

[124] D. V. Talapin, C. T. Black, C. R. Kagan, E. V. Shevchenko, A. Afzali, C. M. Murray, J. Phys. Chem. C 111, 13244 (2007).

[125] J-S. Lee, E. V. Shevchenko, and D. V. Talapin, J. Am. Chem. Soc. 130, 9673 (2008).

[126] F. Gao, Q. Lu, X. Liu, Y. Yan, D. Zhao, Nano Lett. 1743 (2001).

[127] S. Y. Jang, Y. M. Song, H. S. Kim, Y. J. Cho, Y. S. Seo, G. B. Jung, C.-W. Lee, J. Park, M. Yung, J. Kim, B. Kim, J-G. Kim, Y.-J. Kim, ACS NANO 4, 2391 (2010).

[128] C. Toher, and S. Sanvito, Phys. Rev. Lett. 99, 056801 (2007).

[129] N. Sai, M. Zwolak, G. Vignale, M. Di Ventra, Phys. Rev. Lett. 94, 186810 (2005).

[130] G. Vignale and M. Di Ventra, Phys. Rev. B 79, 014201 (2009).

[131] C. Barraud, P. Seneor, R. Mattana, S. Fusil1, K. Bouzehouane, C. Deranlot, P. Graziosi, L. Hueso, I. Bergenti, V. Dediu, F. Petroff, A. Fert, Nature Phys. 6, 615 (2010).

[132] L. Schulz, L. Nuccio, M. Willis, P. Desai, P. Shakya, T. Kreouzis, V. K. Malik, C. Bernhard, F. L. Pratt, N. A. Morley, A. Suter, G. J. Nieuwenhuys, T. Prokscha, E. Morenzoni, W. P. Gillin, A. J. Drew, Nature Mater. 10, 39 (2011).

[133] T. S. Santos, J. S. Lee, P. Migdal, I. C. Lekshmi, B. Satpati, J. S. Moodera, Phys. Rev. Lett. 98, 016601 (2007). 
[134] Z. H. Xiong, Di. Wu, V. Vardeny, S. Jing, Nature 427, 821 (2004).

[135] S. Majumdar, H. S. Majumdar, R. Laiho, R. Osterbacka, J. Alloys Compounds 423, 169 (2006).

[136] V. Dediiu, L. E. Hueso, I. Bergenti, A. Riminucci, F. Borgatti, P. Graziosi, C. Newby, F. Casoli, M. P. De Jong, C. Taliani, Y. Zhan, Phys. Rev. B. 78, 115203 (2008).

[137] S. Sanvito, Nature Phys. 6, 562 (2010).

[138] S. Sanvito, Chem. Soc. Rev. 40, 3336 (2011).

[139] D. Waldron, P. Haney, B. Larade, A. MacDonald, H. Guo, Phys. Rev. Lett. 96, 166804 (2006).

[140] Z. Ning, Y. Zhu, J. Wang, H. Guo, Phys. Rev. Lett. 100, 056803 (2008).

[141] L. Senapati, R. Pati, S. C. Erwin, Phys. Rev. B 76, 0244388 (2007).

[142] H. Dalgleish, G. Kirczenow, Phys. Rev. B 72, 184407 (2005).

[143] S. Mandal, and R. Pati, ACS NANO 6, 3580 (2012).

[144] J. M. D. Coey, M. Venkatesan, C. B. Fitzgerald, A. P. Douvalis, I. S. Sanders, Nature 420, 156 (2002).

[145] G. C. Solomon, C. Herrmann, J. Vura-Weis, M. R. Wasielewski, M. A. Ratner, J. Am. Chem. Soc. 132, 7887 (2010).

[146] P. Ruden, Nature Mater. 10, 8 (2011). 
[147] D. Liu, Y. Hu, H. Guo, X. F. Han, Phys. Rev. B 78, 19307 (2008).

[148] H. -Ch. Mertins, S. Valencia, W. Gudat, P. M. Oppeneer, O. Zaharko, H. Grimmer, Europhys. Lett. 66, 743 (2004). 



\section{Appendix A}

\section{Copyrights}

The copyright permission from The Elsevier for the articles by Subhasish Mandal and Ranjit Pati, Chem. Phys. Lett. 479, 244 (2009). The permission applies to Chapter 4. 


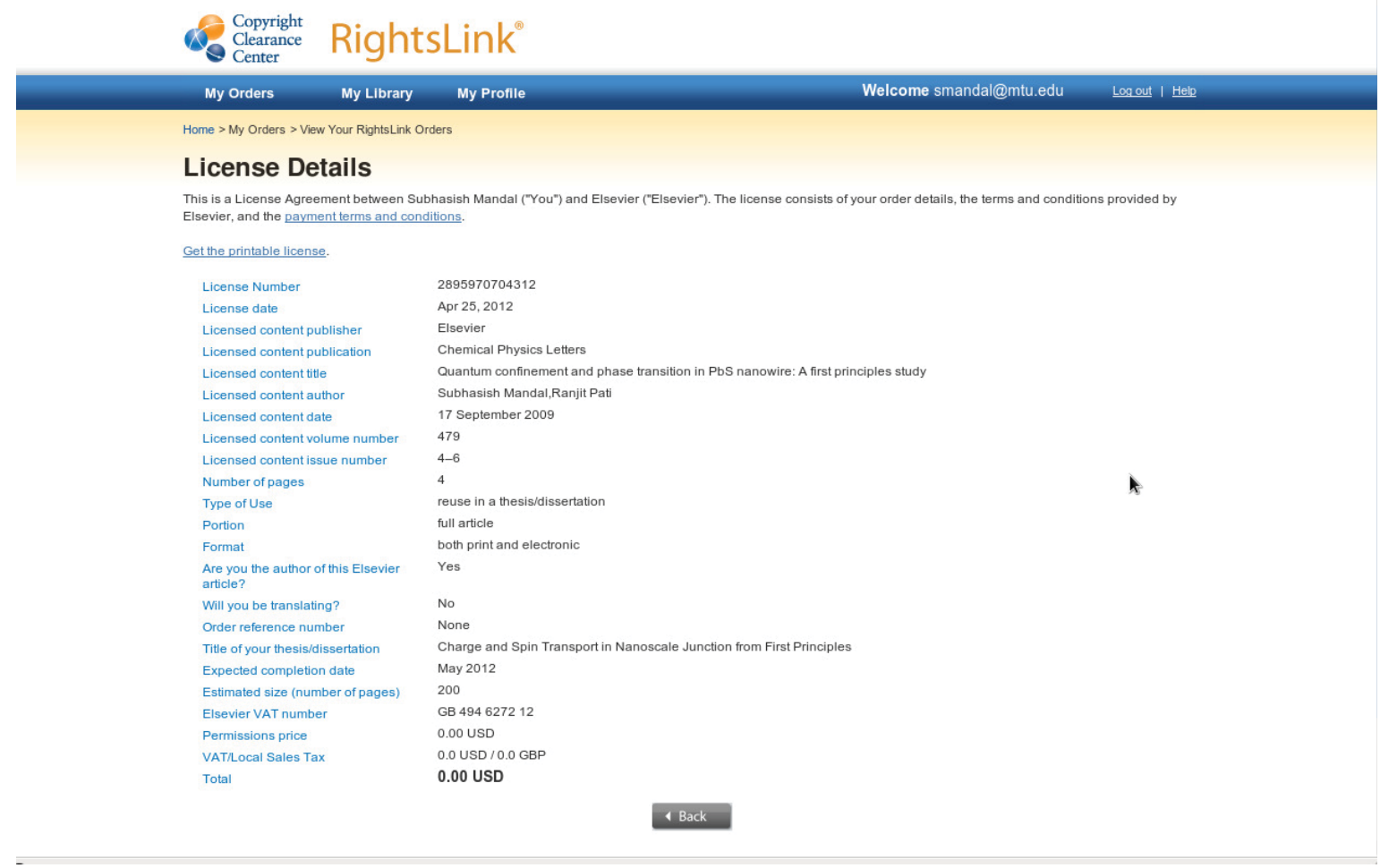

Figure A.1: Copyright permission from The Elsevier for the articles by Subhasish Mandal and Ranjit Pati, Chem. Phys. Lett. 479, 244 (2009). 


\section{Appendix B}

\section{Copyrights}

The copyright permission from The American Physical Society for the articles by Subhasish Mandal and Ranjit Pati, Phys. Rev. B. 83, 195420 (2011) and Phys. Rev. B. 84115306 (2011). The permission applies to Chapter 4 and Chapter 5. 


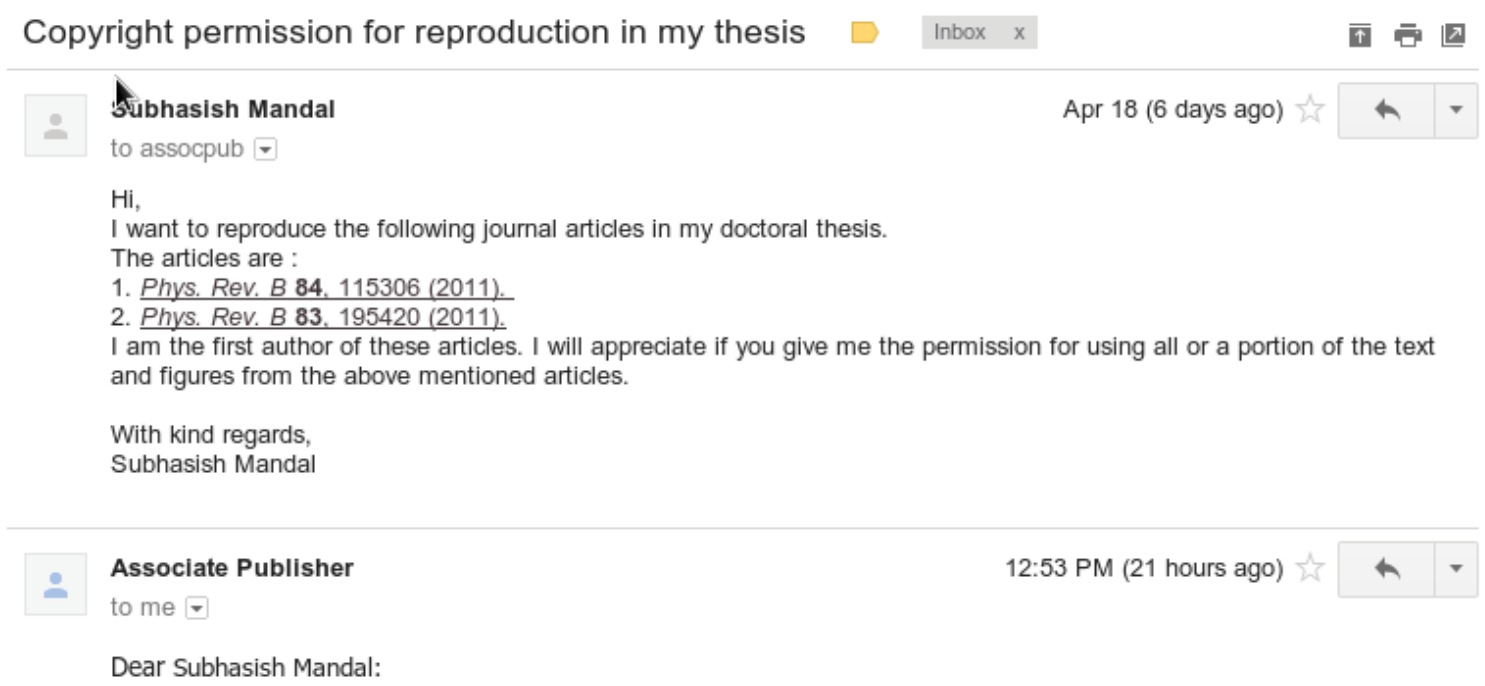

As the author of the APS papers, you have the right to use figures, tables, graphs, etc. in subsequent publications using files prepared and formatted by you or the APS-prepared versions. The appropriate bibliographic citation must be included.

Best wishes,

Melissa Overton

Publications Marketing Coordinator

American Physical Society

http://librarians.aps.org/

Figure B.1: The copyright permission from The American Physical Society for the articles by Subhasish Mandal and Ranjit Pati, Phys. Rev. B. 83, 195420 (2011) and Phys. Rev. B. 84115306 (2011). 


\section{Appendix C}

\section{Copyrights}

The copyright permission from The American Chemical Society for the articles by Subhasish Mandal and Ranjit Pati, ACS Nano 6, 3580 (2012). The permission applies to Chapter 6. 


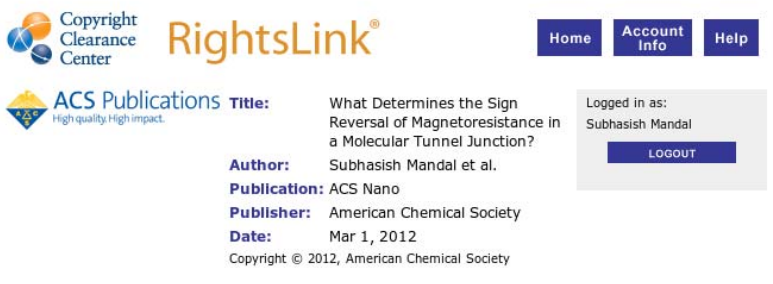

PERMISSION/LICENSE IS GRANTED FOR YOUR ORDER AT NO CHARGE

This type of permission/license, instead of the standard Terms \& Conditions, is sent to you because no fee is being charged for your order. Please note the following:

- Permission is granted for your request in both print and electronic

formats.

- If figures and/or tables were requested, they may be adapted or used in

part.

- Please print this page for your records and send a copy of it to your

publisher/graduate school.

- Appropriate credit for the requested material should be given as follows:

"Reprinted (adapted) with permission from (COMPLETE REFERENCE

CITATION). Copyright (YEAR) American Chemical Society." Insert

appropriate information in place of the capitalized words.

- One-time permission is granted only for the use specified in your request.

No additional uses are granted (such as derivative works or other

editions). For any other uses, please submit a new request.

\section{BACK CLOSE WINDOW}

Copyright o 2012 Copyriaht Clearance Center, Inc. All Rights Reserved. Privacy statement.
Comments? We would like to hear from you. E-mail us at customercare@copyriaht.com

Figure C.1: Copyright permission from The American Chemical Society for the articles by Subhasish Mandal and Ranjit Pati, ACS Nano 6, 3580 (2012). 